Universidad Nacional de La Plata

Facultad de Ciencias Exactas

Departamento de Física

\title{
Magnetoresistividad gigante y propiedades estructurales y magnéticas de los sistemas Fe-Cu y Fe-Au pro- ducidos por aleamiento mecánico
}

Tesis para optar al grado académico de Doctor en Ciencias de la Facultad de Ciencias Exactas, Universidad Nacional de La Plata

Lic. Leandro Martín Socolovsky

Septiembre de 2001 


\section{Resumen}

Hemos producido muestras de $\mathrm{Fe}_{\mathrm{x}} \mathrm{Cu}_{100-\mathrm{x}} \mathrm{y} \mathrm{Fe}_{\mathrm{x}} \mathrm{Au}_{100-\mathrm{x}}$ por molido mecánico en concentraciones variables con el objeto de estudiar el efecto magnetoresistivo gigante y su relación con la microestructura en aleaciones preparadas de esa manera. En el sistema $\mathrm{Fe}_{\mathrm{x}} \mathrm{Cu}_{100-\mathrm{x}}$ efectuamos un estudio del efecto magnetoresistivo gigante en un rango amplio de concentraciones ( $1 \leq x \leq 45 \%$ atómico), ampliando lo conocido hasta ahora en muestras preparadas con los mismos fines. Hemos producido $\mathrm{Fe}_{\mathrm{x}} \mathrm{Au}_{100-\mathrm{x}}$ por primera vez por aleamiento mecánico, en diversas concentraciones (15 $\leq x \leq 30 \%$ atómico). Medimos el efecto magnetoresistivo a $77 \mathrm{~K}$ para cada preparación, y encontramos que el efecto es máximo en $x=20 \%$ para el Fe-Cu, y en $x=25 \%$ para el Fe-Au. No encontramos efecto a temperatura ambiente. Estructuralmente encontramos en ambos sistemas que se forma una solución que tiene la estructura fcc de la matriz $(\mathrm{Cu}$ o $\mathrm{Au})$. En el Fe$\mathrm{Cu}$ el parámetro de red aumenta con la concentración de $\mathrm{Fe}$, mientras que en el $\mathrm{Fe}-\mathrm{Au}$ disminuye. En ambos casos el parámetro de red es diferente a lo que indica la Ley de Vegard, mostrando que los efectos de repulsión química son importantes en estos sistemas. Magnéticamente ambos sistemas son del tipo "cluster-glass", con una concentración de percolación $\mathrm{C}_{\mathrm{p}}=15,9 \pm 3,2 \%$ atómico en el caso del $\mathrm{Fe}-\mathrm{Cu}, \mathrm{y} \mathrm{C}_{\mathrm{p}} \approx 25 \%$ at. en el caso del Fe-Au. El sistema $\mathrm{Fe}-\mathrm{Cu}$ aparece como una dispersión de racimos o clusters de tamaño $\approx 2 \mathrm{~nm}$, de características estructurales y magnéticas similares para todas las concentraciones estudiadas. Los tratamientos térmicos producen una mejora en la relación magnetoresistiva $\Delta \rho / \rho$, pero no en el efecto neto $\Delta \rho$. En el Fe-Cu éste empeora, $\mathrm{y}$ en el $\mathrm{Fe}-\mathrm{Au}$ se mantiene. Este tratamiento favorece, en el sistema $\mathrm{Fe}-\mathrm{Cu}$, la precipitación del $\alpha$-Fe y el $\gamma$-Fe, además de la formación de carburos. En el sistema $\mathrm{Fe}-\mathrm{Au}$ aparece un refinamiento en Fe de los clusters magnéticos, junto a la precipitación de una componente ferromagnética. Ambos sistemas están formados por partículas fuertemente interactuantes. El aleamiento mecánico es una técnica muy apropiada para la preparación de sistemas magnetoresistivos gigantes Fe-metal noble. 


\section{Abstract}

We have produced samples of $\mathrm{Fe}_{\mathrm{x}} \mathrm{Cu}_{100-\mathrm{x}}$ and $\mathrm{Fe}_{\mathrm{x}} \mathrm{Au}_{100-\mathrm{x}}$ by mechanical alloying in varying concentrations with the purpose of study the Giant Magnetoresistance Effect and its relationship with their microstructure in alloys prepared with such technique. We have done on the $\mathrm{Fe}_{\mathrm{x}} \mathrm{Cu}_{100-\mathrm{x}}$ system a study of the giant magnetoresistance effect in a wide range of concentrations $(1 \leq x \leq 45$ atomic $\%)$, spanning the knowledge about Fe-Cu alloys prepared for the same purpose. We have produced Fe-Au alloys by mechanical alloying for the first time, in different concentrations $(15 \leq x \leq 30$ atomic \%). We measured the magnetoresistive effect at $77 \mathrm{~K}$ for every concentration. We found that the maximum effect occurs at $x=20 \%$ for Fe-Cu alloys, and at $x=25 \%$ for Fe-Au alloys. We have not found effect at room temperature. From the structural point of view we found that in both systems a solution with the matrix fcc structure $(\mathrm{Cu}$ or $\mathrm{Au})$ is formed. Lattice parameter increases with concentration in the $\mathrm{Fe}-\mathrm{Cu}$ alloys, while decreases in the Fe-Au alloys. Lattice parameter differs, in both cases, from the Vegard's law expected values, showing that chemical-repulsive effects are important in these systems. Boths systems belongs to the cluster-glass type of magnetism, with a percolation threshold $\mathrm{C}_{\mathrm{p}}=15,9 \pm 3,2$ atomic $\%$ for the Fe-Cu alloys, and $\mathrm{C}_{\mathrm{p}} \approx 25$ at. $\%$ in the case of $\mathrm{Fe}-\mathrm{Au}$ alloys. $\mathrm{Fe}-\mathrm{Cu}$ system consists in a dispersion of clusters of $2 \mathrm{~nm}$ in size, with similar structural and magnetic properties for all the studied concentrations. Thermal treatments produce an enhancement of the magnetoresistive ratio $\Delta \rho / \rho$, but in the effect $\Delta \rho$. It worsens in $\mathrm{Fe}-\mathrm{Cu}$ alloys, and not changes in $\mathrm{Fe}-\mathrm{Au}$ alloys. In the $\mathrm{Fe}-\mathrm{Cu}$ system this treatment favours $\alpha$ - and $\gamma$ - Fe precipitation, and carbides formation. As a consequence of thermal treatments Fe enrichment in the clusters is produced in Fe-Au alloys, besides a precipitation of a ferromagnetic component. Both systems are formed by strongly-interacting particles. Mechanical alloying is a suitable technique for the fabrication of Fe-noble metals giant-magnetoresistive systems. 


\section{Introducción}

\section{Propósito de esta tesis}

El propósito perseguido en esta tesis es la investigación de la magnetoresistividad gigante en aleaciones $\mathrm{Fe}-\mathrm{Cu}$ y $\mathrm{Fe}-\mathrm{Au}$, y su relación con las propiedades estructurales y magnéticas de las mismas. Estudiamos también la influencia del tratamiento térmico en la magnetoresistividad de estas aleaciones.

La posibilidad de la producción de sistemas metaestables que proporciona el aleamiento mecánico atrajo la atención de los investigadores desde los años ochenta. Lo que en principio era una técnica metalúrgica para la producción en masa ${ }^{1}$, dio lugar a su uso como instrumento de fabricación de muestras para la investigación básica. Paralelamente, los materiales nanoestructurados empezaron a recibir la atención de la comunidad académica luego de sucesivos descubrimientos que aunaban nuevos problemas científicos con las aplicaciones tecnológicas. En este contexto, y a partir de los trabajos de Shingu, Eckert y Yavari sobre el sistema Fe-Cu preparado por molido mecánico, el descubrimiento de la magnetoresistividad gigante en sistemas multicapas y del "redescubrimiento" de ese efecto en los sistemas granulares por Chien, es aparecen los primeros trabajos sobre magnetoresistividad gigante (MRG) en sistemas granulares preparados por aleamiento mecánico (MA). Sin embargo, las cuestiones de la dependencia de este efecto con la concentración del elemento magnético, las cuestiones estructurales relacionadas, el estado magnético del Fe en una red fcc, aparecen sin respuestas concluyentes. Además, aunque preliminarmente se suponía que la dilución de un elemento magnético en una matriz no magnética era clave en el efecto MRG, no se habían reportado muchos resultados en el sistema Fe-Metal noble. Como tampoco se había intentado su producción por MA, ésto nos motorizó a intentar la experiencia. El Fe-Au, conocido

\footnotetext{
${ }^{1}$ Se usaba, casi con exclusividad, para producir dispersiones de óxidos en metales.
} 
por ser el mictomagneto prototípico, terminó mostrando el efecto MRG como habíamos buscado.

Nuestras investigaciones en los dos sistemas se describen en esta tesis. En el capítulo I trataremos sobre el sistema Fe-Cu en crudo. Allí comenzaremos dando un panorama de lo que fue investigado en los años previos al comienzo de esta tesis, para continuar con los trabajos contemporáneos a la misma. Los hemos diferenciado así para dar énfasis a que se trata de resultados obtenidos durante, y en algunos casos posteriormente a la realización de algunos de nuestros experimentos, con lo que en algunos casos resultaron guías para nuestro trabajo y en otros como confirmación de nuestros resultados. A continuación de esta base bibliográfica contamos cómo hicimos las muestras en la parte 2. En la sección 3 mostramos los resultados experimentales obtenidos con las diversas técnicas de medida empleadas (Difracción de rayos X, resistividad, magnetoresistividad, susceptibilidad, espectroscopía Mössbauer y dispersión de rayos X a bajos ángulos). En todos los casos damos una explicación parcial a los resultados de una técnica dada, con el ánimo de preparar a la discusión general, lo que se hace en la sección 4. El ánimo de hacer esto es no cerrar los caminos alternativos de interpretación. En el capítulo II hablamos de los tratamientos térmicos que efectuamos sobre el sistema Fe-Cu. Luego de una introducción (sección 1), donde justificamos la orientación de nuestra investigación en ese punto, comentamos la forma en que realizamos esos tratamientos térmicos (sección 2). En la sección 3 damos los resultados de las medidas tomadas (Difracción de rayos X, resistividad, magnetoresistividad, susceptibilidad y espectroscopía Mössbauer). En la sección 4 discutimos los resultados obtenidos. En el capítulo III hablamos del sistema Fe-Au, donde comenzamos (sección 1) por explicar la motivación para hacer experimentos en ese sistema y continuamos con los trabajos previos a esta tesis. En la sección 2 hablamos de cómo preparamos las muestras. En la 
sección 3 presentamos los resultados de las medidas efectuadas (Difracción de rayos X, resistividad, magnetoresistividad, susceptibilidad y espectroscopía Mössbauer), para luego pasar a la sección $\mathbf{4}$ donde hablamos de los tratamientos térmicos sobre este sistema, y de las medidas efectuadas sobre el mismo. A estos capítulos le siguen las Conclusiones, donde ponemos de manifiesto las semejanzas entre los dos sistemas, con el espíritu de rescatar principios generales que sean de utilidad, sino guía, para futuras investigaciones. En tres apéndices desarrollamos puntos que hemos considerado de utilidad para esta tesis, pero que no fueron parte fundamental de la investigación en el sentido de que, en el caso de las técnicas, no hemos introducido modificaciones al uso estándar de las mismas para nuestras muestras. Sí nos ha parecido importante describir con algún detalle sus fundamentos, ya que se trata de técnicas nuevas para el departamento de Física. Es por eso que en el Apéndice B describimos a la dispersión de rayos $\mathbf{X}$ a bajos ángulos (SAXS) y en el Apéndice $\mathbf{C}$ describimos al susceptómetro CA y al SQUID. En el Apéndice A contamos los experimentos que hemos hecho en muestras de $\mathrm{Fe}_{20} \mathrm{Cu}_{80}$ preparadas por aleamiento mecánico para observar el efecto del uso de agente antiadherente (PCA) en la estructura de las mismas, esencialmente la contaminación con carbono. En lo que resta de esta Introducción explicaremos qué es el aleamiento mecánico y qué es la magnetoresistividad gigante y de qué manera la medimos. 


\section{2. ¿Qué es el aleamiento mecánico?}

El aleamiento mecánico (MA) es un conjunto de técnicas que tienen en común que por medios mecánicos proporcionan pequeñas cantidades de energía al sistema en producción. Entre estas técnicas podemos mencionar al molido mecánico (MM) y al laminado en frío. El molido mecánico consiste en colocar el material a tratar en un molino de bolas. El molino, al mover el tambor donde se alojan las bolas y el material, hace que éstas golpeen entre sí y también que las bolas golpeen las paredes internas del tambor. El material queda "ensandwichado" y por tanto va sufriendo las colisiones de las bolas entre si y de ellas con las paredes del tambor. El material sufre así choques continuos que proporcionan pequeños pulsos de energía al material. El sistema se va deformando, fracturando y soldando, hasta alcanzar un equilibrio dinámico entre estos procesos. Por la naturaleza de los choques, la energía es transferida y disipada durante breves períodos de tiempo, lo que no permite al sistema relajar y lograr el equilibrio termodinámico. Gracias a este efecto de "congelamiento", el sistema puede alcanzar estados metaestables. Estos estados bien pueden resultar en fases cristalinas o amorfas, que permanecen hasta temperaturas donde el sistema evoluciona a sus fases estables dentro de la escala de tiempos de este trabajo de tesis. El uso de estas técnicas ha permitido la producción de aleaciones y otros compuestos no obtenibles anteriormente por vías químicas o metalúrgicas usuales. La simpleza y bajo costo del equipamiento es una ventaja a la hora de considerar la economía o el volumen de producción.

El molido mecánico se ha utilizado para la investigación científica desde fines de los '70s. Los años 80 fueron los de despegue y expansión de la técnica, lo que dio cabida a la realización de encuentros especializados como el ISMANAM (International Symposium on Metastable, Mechanically Alloyed, Nanocrystalline Materials). 
Actualmente se clasifican las clases de molinos en "de alta energía" y "de baja energía", aunque no exista un criterio cuantitativo para dicha clasificación. Como veremos más adelante, no es posible comparar en forma directa y precisa los resultados obtenidos con molinos de clases diferentes. Se está intentando modelizar la dinámica de esta técnica, pero ello no ha sido posible en forma completa. Lo que se tiene en claro es que una magnitud relevante es la relación entre la masa de las bolas y la del material a moler. Otra es el tiempo de molido. Otro más es el factor de llenado. La temperatura a la que se encuentra el cuenco es también importante, ya que eso favorece o no el "congelamiento" del material luego del choque ([Pochet 1995]). Si se reemplazan las bolas con cilindros, el resultado es diferente ${ }^{2}$. Lo que es claro es que molinos de tipos diferentes producirán resultados diferentes, dentro de ciertos límites.

Actualmente se propone que el mecanismo que puede explicar ciertos efectos encontrados en el molido mecánico sería el "bombeo de soluto por dislocaciones" [Schwarz 1997]. En este modelo se entiende que el aleamiento mecánico produce inter-

fases limpias entre los metales en proceso de molido, así como una gran densidad de dislocaciones. Según este modelo, existen dos tiempos característicos para las partículas en proceso de

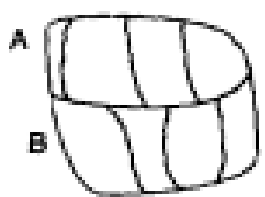

(a)

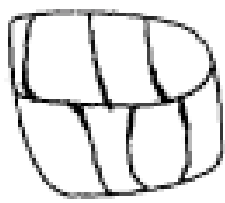

(b)

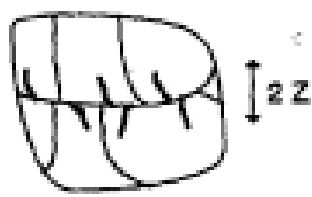

(c)
Fig. 1: Mecanismo de bombeo de soluto por dislocaciones. En (a) dos partículas de polvo A y B se ponen en contacto íntimo y se dislocan por el impacto. En (b) los átomos de $\mathrm{B}$ se difunden por las dislocaciones en $\mathrm{A}$ y viceversa. En (c), a raíz de un nuevo impacto los átomos de soluto quedan atrapados en el otro grano. Tomada de [Schwarz 1997].

molido. En un cierto intervalo de tiempo muy corto (del orden de los microsegundos) la partícula sufre una gran tensión mecánica que supera su resistencia. Se producen dislocaciones que son atractivas para los átomos del soluto, lo que hace que el soluto viaje por a lo largo de las mismas. Como el otro tiempo característico es el que ocurre entre

\footnotetext{
${ }^{2}$ Prof. C. Larica, comunicación privada
} 
dos colisiones sucesivas, y este tiempo es del orden de los segundos, hay tiempo suficiente para que en esas dislocaciones se alcancen soluciones muy saturadas. Un siguiente impacto desplazará esas dislocaciones a una velocidad muy elevada, que hace que el soluto que había entrado en ellas no pueda seguirlas, permaneciendo en el lugar por donde había penetrado. Este proceso, repetido muchas veces, logra la producción de sistemas metaestables como los que nos ocupan.

\section{Tipos de molino}

Como dijimos, existen diversos tipos de molinos, los que, dependiendo de la

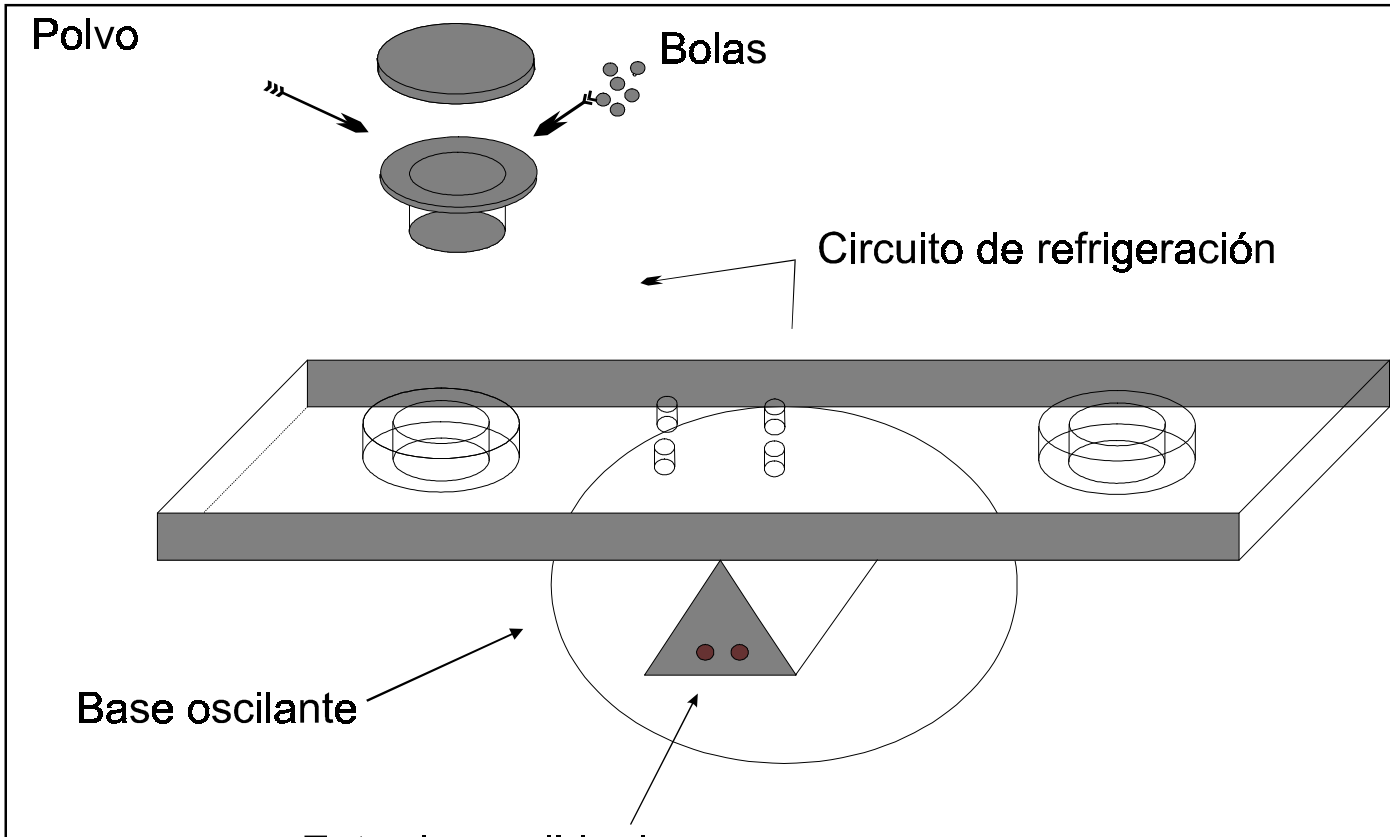

\section{Entrada y salida de agua}

Fig. 2: Esquema del molino vibratorio Nissin gikken NEV-MA8. Los tambores se insertan en los alojamientos ubicados en la plancha. Por esos alojamientos circula agua. La base y la plancha ejecutan movimientos oscilantes que dan por resultado la vibración del tambor. No hemos dibujado los elementos de fijación.

manera que se mueven reciben el nombre de horizontal, de pié, planetario, giratorio o vibratorio. En el último caso, los cuencos cerrados se colocan en ambos extremos de una plancha, la que se apoya por su centro en un eje excéntrico conectado a un motor.

De esta manera la plancha sube y baja, y oscila de un lado a otro (figura 2). 
Normalmente los cuencos tienen una tapa sellada con un "o-ring" que permite efectuar la molienda en atmósfera artificial. En nuestro laboratorio se están haciendo experimentos con cuencos modificados para alojar sensores de presión, conexiones para controlar la atmósfera y compartimientos auxiliares con el fin de efectuar procesamientos secuenciales.

\section{3. ¿Qué es la magnetoresistividad gigante?}

La magnetoresistividad gigante (MRG) es un efecto que aparece en materiales compuestos por metales magnéticos y no magnéticos y que se manifiesta como un cambio negativo e isotrópico en la resistividad eléctrica cuando se le aplica un campo magnético [Chien 1993]. La condición necesaria para la aparición del efecto es la existencia de heterogeneidades estructurales que produzcan dispersión dependiente del espín (spindependent scattering).

La primera evidencia de este comportamiento fue obtenida en 1941 ([Nakhimovitch 1941]) en muestras muy diluídas $A u$ Fe. En los años 50s y 60s se investigó en este y otros sistemas, también con el elemento magnético muy diluído ([Gerritsen 1951, 1953, Rohrer 1968$\left.]^{3}\right\}$.

A partir del trabajo del grupo de Fert [Baibich 1988] sobre magnetoresistencia en multicapas crecidas por epitaxia molecular, se registró una explosión en la investigación de multicapas hechas con un separador de un material no magnético "ensandwichado" entre dos ferromagnéticos. Controlando el espesor de la capa intermedia, los investigadores logran que entre ambas capas ferromagnéticas aparezca un alineamiento antiferromagnético. Al aplicar un campo magnético en la dirección paralela a las capas la magnetización se alinea ferromagnéticamente en esa dirección y la resistencia eléctri-

\footnotetext{
${ }^{3}$ Más referencias en [Nigam 1983]
} 
ca baja considerablemente, independientemente de la dirección en que fue aplicada la corriente.

En 1992 el grupo de Chien reporta ([Xiao 1992]) haber observado magnetoresistividad gigante en sistemas magnéticos granulares preparados por sputtering, en particular el Fe-Cu. Debido al uso de esta técnica, que permite la preparación de aleaciones fuera del equilibrio termodinámico y el control del tamaño de los agregados, fue posible la realización de un buen número de sistemas magnetoresistivos gigantes. Encontraron que la temperatura de un tratamiento térmico ulterior hacía variar la razón magnetoresistiva. Explican que la presencia del efecto GMR es debida a la existencia de entes ferromagnéticos de escala microscópica desalineados, separados una distancia del orden del camino libre medio de los electrones de conducción. El efecto es por tanto dependiente de la densidad y el tamaño de estos entes. En 1993 el mismo grupo presenta una revisión [Chien 1993] de los resultados obtenidos en sistemas magnéticos granulares, y clasifica las diversas clases de magnetoresistividad. En este trabajo predice también que el máximo efecto magnetoresistivo se encuentra para una concentración aproximada de 25 $\%$ en volumen.

Para los sistemas magnéticos granulares como los que nos ocupan el desarrollo teórico no ha sido tan pronunciado como en los sistemas magnéticos multicapas. Se han hecho teorías para describir el comportamiento magnetoresistivo gigante en los sistemas granulares, mayormente basados en los desarrollos para las multicapas. Por eso, describiremos brevemente el modelo para las multicapas, para de allí pasar a los sistemas granulares. La teoría hoy aceptada es la de la existencia de canales de gran conductividad para los electrones de conducción. A partir de la idea de Mott se pueden separar a los electrones de conducción en dos clases: los que tienen su espín paralelo a la magnetización, y aquellos que están antiparalelos a ella. Con ello establecemos dos sub-bandas: 
Una en la dirección de la magnetización (llamada "spin-up"), y la otra antiparalela (llamada "spin-down”). Así, existirán dos corrientes (de acuerdo a Fert, ver [Rossiter 1991]). De las diferentes dispersiones que sufren los electrones de conducción, la que da origen al efecto magnetoresistivo gigante es la que depende del espín. En el caso de las multicapas tenemos al menos un "sándwich" de un material no magnético entre dos capas o láminas de materiales magnéticos. Distinguimos dos situaciones: cuando hacemos circular una corriente perpendicular al sándwich (CPP, por Current Perpendicular to the Plane); y la otra, cuando hacemos circular corriente en una dirección en el plano del sándwich (CIP, por Current In the Plane). El campo magnético se aplica en el plano del sándwich. En un experimento de magnetoresistividad CIP en un sistema cuyo orden espontáneo es antiferromagnético, en la situación de campo aplicado nulo la corriente de electrones "spin-up” no será dispersada en una de las capas magnéticas, pero sí en la otra. Y lo mismo para la corriente "spin-down": será dispersada en la primera, pero no en la segunda. El resultado neto es que ambas corrientes sufren la misma dispersión. Pero al aplicar un campo que cambie la orientación relativa de ambas láminas y las oriente en una misma dirección, una de las corrientes no se verá dispersada por su espín, con lo que la resistividad será menor que cuando no se aplicó un campo externo. Este efecto no ocurre, como se ve, si las capas están muy lejos una de otra, con lo que el electrón no llega a sensar el alineamiento de las capas. Esta distancia es el camino libre medio $\Lambda$ de los electrones de conducción. El efecto es mayor cuanto más grande es el alineamiento entre las capas. Los sistemas con un alineamiento antiferromagnético dan el mayor efecto, pero éste también existe si en ausencia de campo las magnetizaciones de las capas ferromagnéticas están distribuidas aleatoriamente en sus direcciones fáciles. Como vemos, este efecto existe por la dispersión dependiente del espín. La dispersión aparece por la rugosidad de las interfaces. Una interfaz perfectamente plana sólo producirá una 
reflexión de tipo especular, y no el efecto de dispersión [Baibich 1996]. Esto ha sido probado experimentalmente, dentro de límites reales (no es posible obtener en laboratorio una interfaz perfectamente plana). En el caso de un experimento de magnetoresistividad CPP la magnitud relevante, según Valet y $\mathrm{Fert}^{4}$, es la longitud de difusión

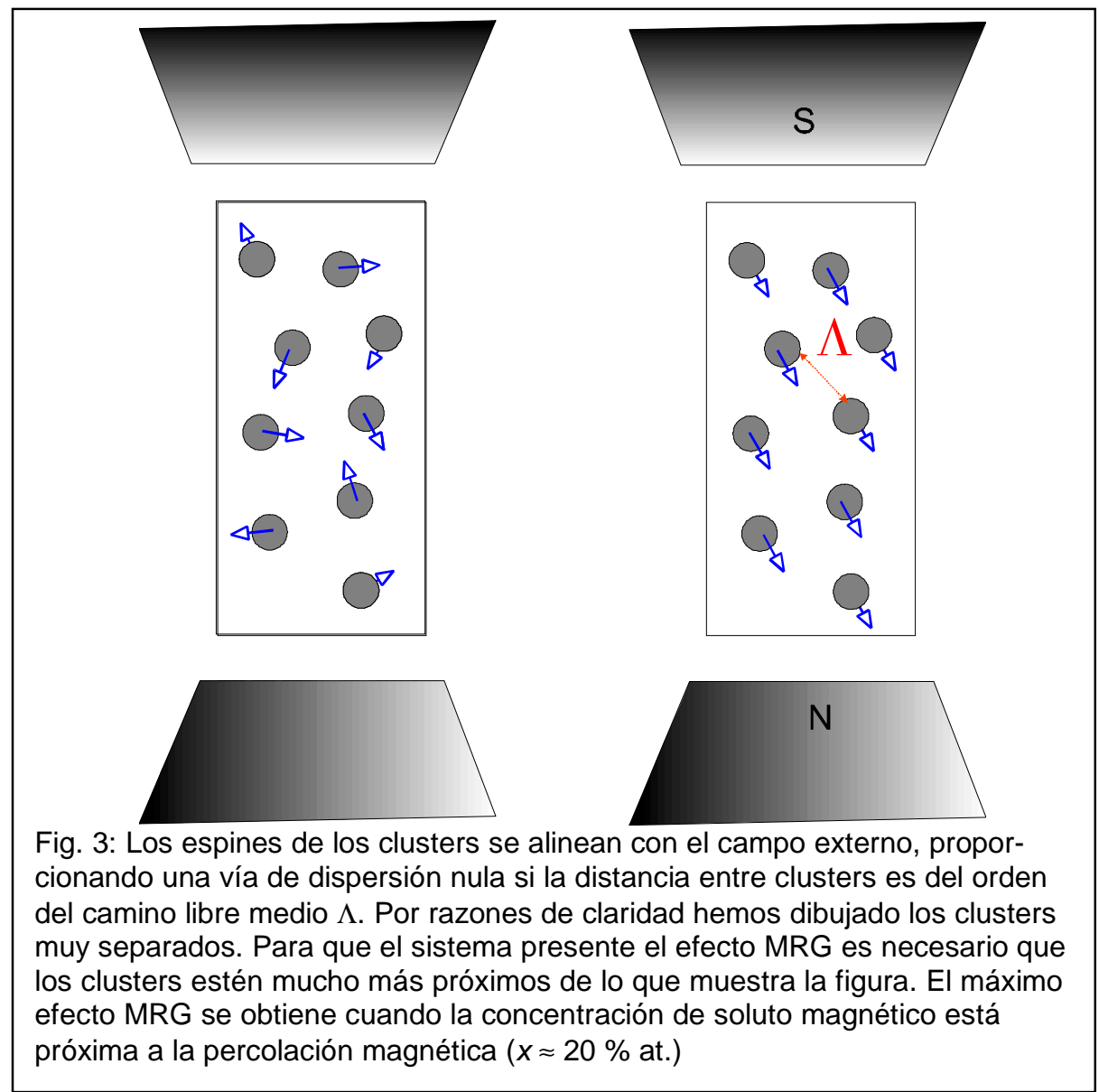

del espín $\lambda_{\text {sdl }}$, que está relacionada con la distancia promedio que viaja el electrón antes que su espín se reoriente.

En el caso de los sistemas magnéticos granulares ocurre algo similar, sólo que al tener un conjunto de entes magnéticos inmersos en una matriz no magnética, ese camino libre medio debe ser del orden de la distancia entre esos entes (racimos o clusters, en lo que sigue de esta tesis). Así, con campo aplicado cero, la dispersión dependiente del es-

\footnotetext{
${ }^{4}$ [Levy 1994] página 394
} 
pín ocurre para todos los electrones por igual. Al aplicar un campo hay una alineación (parcial a campos pequeños) de los espines de los clusters. Eso hace que para los electrones con esa orientación haya menos dispersión, y por ende menos resistividad. El máximo efecto lo obtenemos al alinear completamente a los espines de esos racimos en una dirección (ver figura 3). Es claro entonces que, además de la distancia entre racimos, las cantidades importantes son el tamaño, el momento magnético de los elementos constitutivos del racimo o cluster, y la rugosidad del mismo. El control de estos parámetros se realiza mediante la concentración, los materiales utilizados, el control de algunos de los parámetros de la técnica de fabricación (como la temperatura del sustrato, la presión del gas en la cámara de sputtering, el tiempo de molido o la relación de masas entre bolas y material en el molido mecánico) y los tratamientos térmicos posteriores.

Se toma ${ }^{5}$ como medida del efecto a la cantidad

$$
\frac{\Delta \rho}{\rho}=\frac{\rho(H)-\rho(0)}{\rho(0)} \times 100
$$

donde $\rho(H)$ y $\rho(0)$ son las resistividades con y sin campo aplicado $H$, respectivamente. Esta cantidad es conocida como razón magnetoresistiva.

\section{Procedimiento para las medidas de magnetoresistividad}

En esta parte describiremos el procedimiento utilizado para las medidas de magnetoresistividad que presentamos en esta tesis.

Medimos magnetoresistividad sobre lingotes de forma paralelepípeda recta rectangular de 0,5 mm de espesor, 1,68 $\mathrm{mm}$ de ancho y 2,8 ó 4,5 mm de largo. Estos lingotes los obtuvimos al compactar el material con una prensa en una matriz de acero duro. Medimos la magnetoresistividad utilizando el conocido método de las cuatro puntas. Para ello seguimos el siguiente procedimiento: colocamos al lingote sobre un cabezal. Este cabezal era una plancha de cobre, envuelto en una cinta de Teflon a fin de aislarlo del 
lingote. Colocamos una pieza de acrílico que se atornillaba al cabezal, y de esta manera la muestra quedaba entre cabezal y pieza de acrílico (ver figura 4). Cuatro electrodos (confeccionados en bronce fosforoso) anclados en la pieza de acrílico presionaban al lingote contra el cabezal. Esta disposición permite flexibilidad al contacto y evita posi-

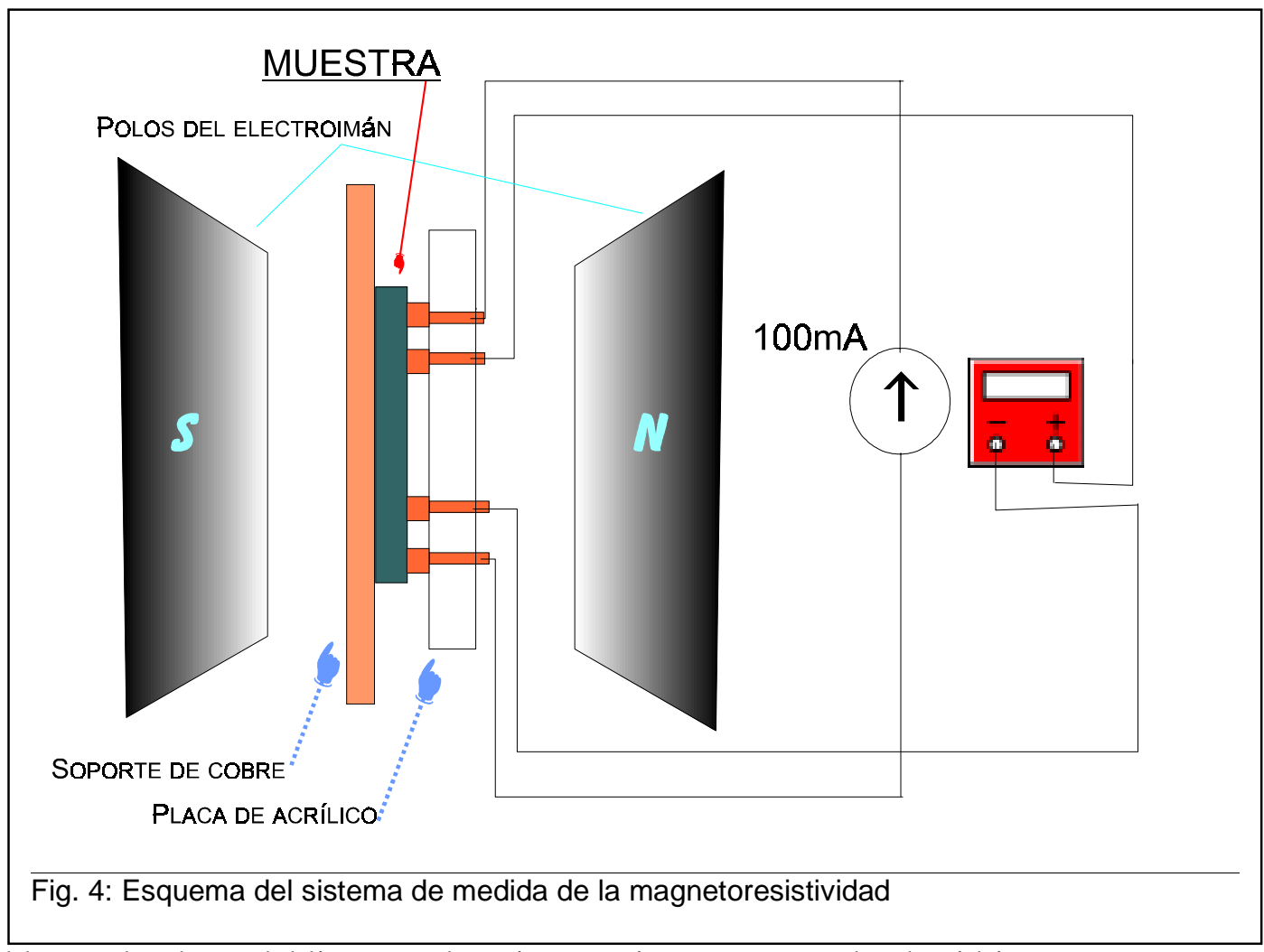

bles quebraduras del lingote. El conjunto se inserta en un tubo de vidrio grueso, que a su vez colocamos entre los polos de un electroimán de paso controlable. Con una fuente de corriente de $100 \mathrm{~mA}$ alimentamos los electrodos externos de la muestra, y registramos la caída de tensión entre los electrodos internos con un tester programable. Mediante una PC tomamos los datos de la caída de tensión para cada campo magnético aplicado. La PC además enviaba una señal de inversión de la polaridad a la fuente de corriente. Como el electroimán cambiaba el campo magnético a intervalos regulares, esto permitía tomar a un campo dado, una lectura de la caída de tensión con la corriente en un sentido

\footnotetext{
${ }^{5}$ Algunos autores prefieren utilizar $\rho\left(H_{\text {máx }}\right)$ en lugar de $\rho(0)$.
} 
y otra lectura con la corriente en el sentido inverso. Esas dos lecturas se promediaban, a fin de eliminar la parte debida al efecto Peltier. La PC almacenaba ese promedio y el campo aplicado en un archivo. El tubo de vidrio nos permitió hacer medidas a $77 \mathrm{~K}$, ya que podíamos sumergir el conjunto en nitrógeno líquido. El procedimiento de medida seguido consistía en elevar el campo hasta el máximo, y allí comenzar las lecturas para luego ir disminuyendo el campo a cero y luego aumentarlo en el sentido opuesto. Una vez alcanzado el máximo campo inverso finalizábamos la medida. El campo magnético máximo aplicado fue de 1,4 T. Todas las medidas a $77 \mathrm{~K}$ fueron tomadas luego de enfriar hasta esa temperatura sin campo aplicado. 


\section{Capítulo I}

\section{El sistema $\mathrm{Fe}-\mathrm{Cu}$}

\section{Introducción}

1. Trabajos previos

2. Preparación de las muestras

3. Medidas

1. Magnetoresistividad

2. Difracción de rayos $X$

3. Resistividad

4. Medidas de susceptibilidad magnética

5. Espectroscopía Mössbauer

6. Dispersión de rayos $X$ a bajos ángulos

\section{Discusión y conclusiones}




\section{Introducción}

El sistema $\mathrm{Fe}-\mathrm{Cu}$ ha sido objeto de interés de la ciencia de materiales desde antaño. Al tener este sistema una entalpía de formación positiva $(13 \mathrm{~kJ} / \mathrm{mol}$ [de Boer

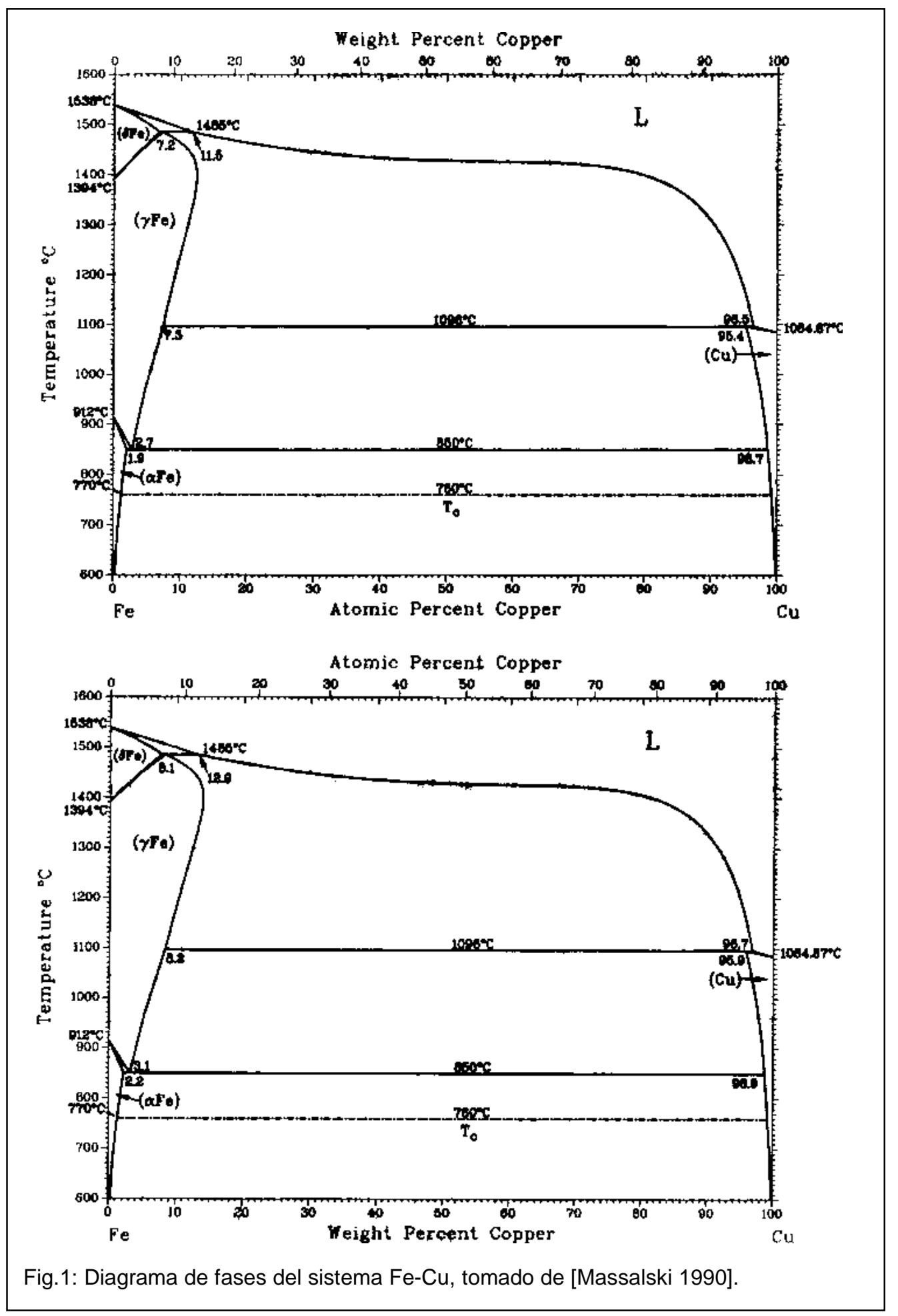


1989]) las solubilidades mutuas son muy pequeñas $\left(3,5 \%\right.$ a $1096{ }^{\circ} \mathrm{C}$ para $\mathrm{Fe}$ en cobre y 6,7 \% at. de $\mathrm{Cu}$ en hierro [Massalski 1990]), sólo es posible mezclarlos por métodos fuera del equilibrio termodinámico, como el enfriado rápido desde el estado líquido (fast quenching), la evaporación y deposición sobre un sustrato frío (sputtering o epitaxia molecular MBE). Desde hace algunas décadas es conocido que es posible unir ambos materiales por prensado en frío. Tanto ésta como el molido mecánico (MM) constituyen técnicas de aleamiento mecánico que permiten el mezclado de este sistema ([Shingu 1991]).

\subsection{Trabajos previos}

En esta sección daremos cuenta de los trabajos realizados por otros autores en el sistema $\mathrm{Fe}-\mathrm{Cu}$ preparado por aleamiento mecánico ${ }^{1}$, en particular de aquellos donde se utilizó un molino de bolas. Como nuestra tesis se desarrolla sobre los sistemas donde el hierro es el componente minoritario, nuestra revisión se detendrá en aquellos donde la concentración $x$ de Fe es de 50 \% atómico o menor.

El grupo de Shingu realizó por primera vez el aleamiento mecánico del sistema $\mathrm{Fe}-\mathrm{Cu}$ en todo el rango de concentraciones [Shingu 1990, Uenishi 1992]. Ellos encontraron $^{2}$ que el sistema era fcc hasta aproximadamente $60 \%$ atómico de Fe, y bcc para cerca de $x=80 \%$ at. Observaron también que el parámetro de red de la zona fcc es más alto que el que correspondería a la ley de Vegard, y que se incrementa con la concentración de Fe. Ven que el momento magnético por átomo no cambia mucho en la zona rica en Fe, pero que sí lo hace en la zona de cobre mayoritario, donde decrece hasta hacerse nulo alrededor de un $80 \%$ atómico de $\mathrm{Cu}$. Efectuaron un análisis térmico, donde se vió la existencia de dos picos exotérmicos, uno alrededor de $453 \mathrm{~K}$ y el otro a

\footnotetext{
${ }^{1}$ En algunos casos mencionaremos resultados del sistema preparado por otros medios. En ese caso aclararemos qué tipo de técnica fue utilizada.

2 Para las muestras obtenidas por molido mecánico
} 
$630 \mathrm{~K}^{3}$. Para las muestras tratadas por encima de esta última temperatura los difractogramas de rayos X mostraron la reaparición del pico bcc. En [Shingu 1990] se afirma que la fase metaestable se produce por la difusión mutua entre los elementos, desde los bordes de grano al interior de los mismos. El potencial químico de los átomos en los bordes de grano es superior al de aquellos en la fase metaestable, por lo que el proceso de difusión ocurrirá en el sentido de la formación de la aleación.

En una serie de trabajos ([Eckert 1992, Eckert 1993]), un grupo de California analizó exhaustivamente, por difracción de rayos X y calorimetría principalmente, la evolución de los parámetros estructurales con el tiempo de molido y con la temperatura del tratamiento térmico. Confirmaron la existencia de las zonas fcc y bcc puras $(x<60$ $\%$ at. y $x>80 \%$ at., respectivamente). Vieron también que en la zona intermedia hay coexistencia de ambas fases. En uno de estos trabajos ([Eckert 1993]) se vió que al calentar las muestras de fase fcc, primero liberaban tensiones para luego, con muy pequeño crecimiento de grano, efectuar la separación en las fases de equilibrio. Una vez separadas las fases, los granos comenzaban a crecer. En este mismo trabajo se determinó el tamaño de los granos, que en la región con $x<60 \%$ (fase fcc) tienen entre 9 y $20 \mathrm{~nm}$.

En el mismo 1992, el grupo de Yavari [Yavari 1992] reportó la fabricación de una solución sólida fcc Fe-Cu. Realiza medidas de DRX, y observa que los picos del hierro y el cobre permanecen con intensidades relativas casi constantes al principio del molido. Cuando alcanzan un tamaño de alrededor de $10 \mathrm{~nm}$, el pico bcc del Fe comienza a desaparecer, hasta que no quedan rastros detectables del mismo. En ese trabajo se discute la idea de [Shingu 1990] acerca de que la energía almacenada en los bordes de grano o en las interfases es la que produce el aleamiento. Hace una estimación

\footnotetext{
${ }^{3}$ Para las muestras ricas en cobre, que es la zona que nos interesa
} 
de las energías involucradas, y concluye que por sí solo, el mecanismo anterior no da cuenta de la energía necesaria para sobreponerse a la energía positiva de formación. En su lugar, propone que el trabajo mecánico genera (por sucesivos angostamientos de los granos del metal bcc) pequeños fragmentos, con la suficiente energía libre como para disolverse en los granos de $\mathrm{Cu}$. El mezclado mecánico dispersa estos átomos en la matriz del cobre. Estima el tamaño de estos fragmentos en $1 \mathrm{~nm}$. En este trabajo se muestran fotografías de microscopía de barrido (SEM) y de transmisión (TEM), que apoyarían lo antedicho. Aquí se sugiere que esos fragmentos bcc pueden hacer un seudomorfismo con la matriz del cobre, adoptando una estructura fcc. El trabajo también reporta una medida de magnetización vs. temperatura, donde se ve una caída de la misma, hasta hacerse nula a $500 \mathrm{~K}$, para luego (alrededor de $600 \mathrm{~K}$ ) aumentar hasta tener la magnetización del $\alpha-F e$.

También en 1992 Ma, Atzmon y Pinkerton investigan las propiedades termodinámicas y magnéticas en función de la concentración [Ma 1993]. Las medidas estructurales son similares a las reportadas en los trabajos previos. Este grupo mide las entalpías de formación a través de las medidas hechas por calorimetría diferencial. De la comparación de estos resultados con un modelo para la entalpía de formación donde se tiene en cuenta un término magnético, se ve que para la zona bcc éste es adecuado, pero para la zona de nuestro interés el desacuerdo es todavía alto (hasta unos $2,5 \mathrm{~kJ} / \mathrm{mol}$ ). Los autores argumentan que las expresiones de la energía libre utilizadas corresponden a situaciones del equilibrio termodinámico, así que para estos sistemas no son completamente apropiadas. Las medidas de magnetización vs. temperatura muestran una transición ferromagnética-paramagnética y luego ferro de vuelta. Las temperaturas de Curie son menores a las del $\alpha$-Fe. 
La primera investigación detallada por efecto Mössbauer fue realizada por Jiang, Gente, Gonser y Bormann [Jiang 1993]. Allí monitorean la formación de la aleación $\mathrm{Fe}_{50} \mathrm{Cu}_{50}$ con el tiempo de molido, y reportan que el producto final es una distribución ancha de campos hiperfinos, de promedio 20,59 T, más una muy pequeña cantidad de Fe bcc y Fe fcc. Analizan también la evolución de las poblaciones de cada fase con la temperatura del tratamiento térmico.

En una réplica [Hernando 1993] al trabajo de Yavari, un grupo español analiza en particular el comportamiento magnético con la temperatura de la aleación de Yavari. Encuentran que luego de la segregación del $\alpha$-Fe, la magnetización del sistema es menor que la de la aleación Fe-Cu original. Esto da pié para sugerir que parte del hierro se encuentra o bien como $\gamma$-Fe sin orden magnético, o bien en un estado no magnético. A su vez, Yavari contesta [Yavari 1993] que la precipitación de $\gamma$-Fe en las muestras tratadas térmicamente es posible, y es consistente con una descomposición espinodal ${ }^{4}$ del sistema.

A partir de estos trabajos sobre el $\mathrm{Fe}-\mathrm{Cu}$, la atención fue puesta en las propiedades de la aleación equiatómica, y sobre el mecanismo de descomposición de la misma [Crespo 1993]. De la espectroscopía Mössbauer obtienen un campo promedio de 22,3 T. Ven también que con el aumento en la temperatura del tratamiento térmico la temperatura de Curie aumenta hasta más allá de $700 \mathrm{~K}$, con un comportamiento tipo $\alpha$ Fe. Reportan también que el momento magnético de las aleaciones tratadas a 723 y 923 K tomado a $5 \mathrm{~K}$ es menor en un $20 \%$ al de la aleación "cruda". En la muestra tratada a $723 \mathrm{~K}$ se ve un comportamiento anómalo en el campo coercitivo, ya que presenta un mínimo al bajar la temperatura. Estiman que algunos cristales, luego del tratamiento

\footnotetext{
${ }^{4}$ La descomposicón espinodal es un mecanismo típico en las aleaciones metaestables o inestables [Cahn 1968]
} 
térmico a alta temperatura, tienen una composición $\mathrm{Fe}_{17} \mathrm{Cu}_{83}$, tomando en cuenta que la composición con $x<20$ no era magnética. La explicación que encuentran es que la descomposición de la aleación metaestable no puede ser directamente fcc $\mathrm{Fe}-\mathrm{Cu} \rightarrow \mathrm{fcc}$ $\mathrm{Cu}+\mathrm{bcc} \mathrm{Fe}$, sino primero a

$$
\text { fcc } \mathrm{Cu}_{\text {rica }} \mathrm{Fe}_{\text {pobre }}+\text { fcc } \mathrm{Cu}_{\text {pobre }} \mathrm{Fe}_{\text {rica }} \text {, }
$$

para luego ser

$$
\text { fcc } \mathrm{Cu}_{\text {rica }} \mathrm{Fe}_{\text {pobre }}+\text { bcc } \mathrm{Cu}_{\text {pobre }} \mathrm{Fe}_{\text {rica. }} \text {. }
$$

El producto final quedaría como

$$
\mathrm{bcc} F e+f c c \mathrm{Cu}+\mathrm{fcc} F \mathrm{.}
$$

Ese hierro fcc es no magnético, y bien puede estar como átomos dispersos en el $\mathrm{Cu}$, o como $\gamma$-Fe (también no magnético), o ambos al mismo tiempo. Los autores se inclinan a pensar que se trata mayormente de $\gamma$-Fe. El comportamiento del campo coercitivo con la temperatura de la muestra tratada a $723 \mathrm{~K}$ así como la gran diferencia que aparece en la magnitud de ese campo a temperatura ambiente entre esa muestra tratada y la de la muestra cruda son explicados con el argumento de que la muestra se descompuso espinodalmente. Así, hay pequeños granos bcc de hierro (monodominio) inmersos en granos fcc ricos en cobre. Existe una dispersión en las temperaturas de Curie de los granos fcc, que serían ferromagnéticos a bajas temperaturas, y mayormente paramagnéticos a temperatura ambiente. Entonces, a muy bajas temperaturas los granos bcc pueden acoplarse vía la matriz fcc, y por tanto el campo coercitivo cae con la temperatura. Esto ocurriría hasta que los granos fcc devienen paramagnéticos, inhibiendo así el orden ferromagnético. Las partículas bec quedarían aisladas, y así la coercitividad se incrementaría. A continuación, el grupo español presenta más resultados sobre la evolución de las propiedades magnéticas con la temperatura del 
tratamiento térmico [Crespo 1994]. Se concluye allí que la descomposición espinodal da lugar a una dispersión en las temperaturas de Curie de unos $1000 \mathrm{~K}$.

Kuhrt y Schulz publican en 1993 [Kuhrt 1993] resultados sobre medidas estructurales y magnéticas en las concentraciones $x=10,20,30$ y $50 \%$ at.Un resultado interesante fue reportado por el grupo de Chien en 1993 [Ambrose 1993], donde vio que el campo coerciTIvo aumentaba con el tiempo de molido, hasta llegar a un máximo, correspondiente al momento en que comenzaba la desaparición de la señal DRX correspondiente a la fase bcc. Luego caía hasta hacerse casi nulo. En el trabajo de Chien se reportó que la temperatura de Curie aumentaba con la concentración, pero siempre a valores muy por debajo de los $1040 \mathrm{~K}$ del $\alpha$-Fe.

En 1994 Huang, He y Wu publicaron un trabajo [Huang 1994] donde se exhiben fotos de microscopía de muy alta resolución (HRTEM). Es posible ver que en las zonas de soldadura hay cristales muy pequeños y zonas nanométricas con estructura amorfa. En ese trabajo concluyen que el mezclado se produce por interdifusión en estado sólido y que la elevación de la energía libre del sistema se debe al almacenamiento de energía en defectos de la red y en energía interfacial.

Una confirmación adicional a la progresiva incorporación del hierro en la matriz del cobre fue hecha en 1994 [Macrí 1994] $]^{5}$, en una muestra $\mathrm{Fe}_{50} \mathrm{Cu}_{50}$. Esta incorporación se siguió por difracción de neutrones ${ }^{6}$ y por DRX. La desaparición del pico principal bcc observada por DRX ocurrió a las 8 horas de molido. Pero por difracción de neutrones ello aconteció a las 16 horas, si bien a las 8 horas apenas era visible un hombro. También se reportan resultados de espectroscopía Mössbauer, que,

\footnotetext{
${ }^{5}$ Un detalle de estos resultados se hallan también en [Macrí 1995a].

${ }^{6}$ La difracción de neutrones es muy apropiada para este caso, donde en la DRX los picos principales del $\alpha$-Fe y del Cu se encuentran muy próximos y se pueden solapar por el ensanchamiento de línea producido por la disminución del tamaño de grano, propio del molido mecánico. En la difracción de neutrones el hierro es más visible que en la DRX.
} 
en acuerdo con Jiang et al., dan cuenta de la presencia de tres sitios distintos para el Fe: uno representado por un sextete ensanchado (zonas ricas en Fe), otro por un doblete (zona rica en $\mathrm{Cu}$ ), y un singlete (posiblemente $\gamma$-Fe). Finalmente siguen, por espectroscopía Mössbauer, la evolución del sistema con la temperatura de tratamiento térmico.

A fines de 1994 fue publicado el primer trabajo sobre el efecto magnetoresistivo gigante en una aleación Fe-Cu preparada por MM [Saxena 1994]. La composición era $\mathrm{Fe}_{15} \mathrm{Cu}_{85}$. Ven que el efecto es mayor cuanto menor es la temperatura de medida. Realizan un tratamiento térmico a $573 \mathrm{~K}$, que produce una mejora en la razón magnetoresistiva, la cual empeora si se incrementa la temperatura de tratamiento. Un trabajo de 1994 sugiere que el tratamiento térmico precipita una fase fcc rica en Fe, y que ésta es ferromagnética [Drbohlav 1994].

Algunos trabajos donde se comparan las características de aleaciones Fe-Cu con otras Co-Cu y mezclas Fe-Co en Cu se presentan en 1995 [Macrí 1995b, Elkalkouly 1995, Yermakov 1995]. En este último se presentan mediciones de magnetoresistividad en una muestra $\mathrm{Fe}_{20} \mathrm{Cu}_{80}$ y el efecto del tratamiento térmico, que es el de disminuir la MRG.

En [Elkalkouly 1995] se presentan algunas medidas de estructura fina de absorción de rayos $\mathrm{X}\left(\mathrm{EXAFS}^{7}\right)$. Se encuentra que el entorno del Fe es el mismo que "ve" el $\mathrm{Cu}$, con lo que se afirma que la estructura es toda fcc. Pese a las dificultades experimentales (debido a que los números atómicos del $\mathrm{Fe}$ y el $\mathrm{Cu}$ son bastante próximos) es posible concluir que no existen entornos bcc. Lo que se afirma también es

\footnotetext{
${ }^{7}$ Extended X-ray Absorption Fine Structure: Es una técnica que consiste en el análisis del espectro de absorción de rayos $\mathrm{X}$ en zonas próximas a los bordes de absorción atómica de un elemento presente en la muestra. En el detalle de este espectro es posible observar algunas oscilaciones relacionadas con el entorno químico y topológico en que se encuentra el elemento.
} 
que el entorno para el $\mathrm{Cu}$ en las muestras molidas está desordenado. También en [Crespo 1995] se presentan resultados EXAFS similares al anterior, y además se confirma la progresiva aparición de la fase bcc con la temperatura de tratamiento térmico.

Un grupo australiano publica en 1995 dos trabajos sobre las propiedades magnéticas [Ding 1995] y magnetoresistivas [Eilon 1995] de la aleación $\mathrm{Fe}_{25} \mathrm{Cu}_{75}$. En esos trabajos los autores explican el comportamiento magnético de la aleación por la presencia de clusters magnéticos que interactúan entre sí, y que serían superparamagnéticos. Esos clusters serían zonas ricas en Fe. Sostienen que el tratamiento térmico (a $473 \mathrm{~K}$ ) segrega la fase alfa del hierro, a partir de átomos dispersos en la matriz fcc del $\mathrm{Cu}$. Observan también que el efecto magnetoresistivo no cambia mucho con la temperatura de medida, así como tampoco hay diferencias significativas en el efecto entre la muestra "cruda" y la tratada térmicamente.

\section{Algunos trabajos contemporáneos a esta tesis}

Un grupo de Louisiana publica en 1996 ([Schilling 1996]) más resultados EXAFS, que confirman lo visto anteriormente, acerca del aleamiento a nivel atómico del Fe y el $\mathrm{Cu}$. Comparan con la aleación Fe-Ag [Ma 1997], que permanece prácticamente sin reaccionar ante el trabajo mecánico.

Un grupo de Muroran reporta en [Ueda 1996] estudios de magnetización, DRX y magnetoresistividad en concentraciones de entre 10 y $90 \%$ at., en muestras "crudas" y tratadas térmicamente a 523, 573 y $673 \mathrm{~K}$. Ven que la magnetización es nula para $x=20$ $\%$ at. y que para la muestra cruda el máximo en el efecto MRG se halla para $\mathrm{x}=30 \%$ at., pero cuando se trata térmicamente ese máximo se desplaza hacia $25 \%$ at. También encuentran que el sistema presenta un comportamiento tipo "spin-glass" reentrante para x entre 18 y $28 \%$. 
También en 1996 aparece un estudio por TEM del $\mathrm{Fe}_{50} \mathrm{Cu}_{50}$ [Mazzone 1996]. Encuentran una variedad de estructuras, incluso algunas con una estructura laminar, la que atribuyen a una descomposición espinodal previa al tratamiento térmico. También ven que los granos ricos en Cu tienen una concentración de Fe de un $30 \%$.

Ese mismo año se reporta en [Huang 1996] que el proceso de aleado mecánico en este sistema está gobernado por el "atomic shear event" y el proceso de difusión inducido por el esfuerzo constante. El trabajo se completa con otro [Huang 1998] donde los autores ven que el tamaño de grano cae a $20 \mathrm{~nm}$ y el contenido de $\mathrm{Cu}$ en los granos (aún bcc) ricos en Fe crece al $20 \%$. Esto hace que la fase bcc se vuelva inestable, y que esos granos ricos en Fe hagan una transformación martensítica hacia la fase $\mathrm{fcc}^{8}$. De esta manera quedan granos de dos clases: $\mathrm{los} f c c-\mathrm{Fe}_{\text {ricos }} \mathrm{Cu}$ y los fcc-Cu $\mathrm{Cu}_{\text {ricos }} \mathrm{Fe}$. El trabajo mecánico posterior promueve la interdifusión, facilitada por la similitud en el parámetro de red $^{9}$ que adquieren los granos ricos en Fe y los ricos en Cu. Esta interpretación es compatible con los resultados de Jiang por Mössbauer y XRD.

El grupo español reporta, en 1997, algunos resultados de la caracterización por DRX y ME de muestras con $x=5,10$ y $20 \%$ atómico ([Barro 1997]).

En 1998 Kaloshkin et al. encuentran que la presencia de oxígeno durante el molido facilita la amorfización y por ende la formación de la solución sólida [Kaloshkin 1998].

\footnotetext{
${ }^{8}$ Se ha visto que la transformación martensítica baja su temperatura por adición de pequeñas cantidades de $\mathrm{Cu}$ a la fase bcc.

${ }^{9}$ Para el $\gamma$-Fe es $0,36467 \mathrm{~nm}$ y para el Cu es $0,36150 \mathrm{~nm}$.
} 


\section{Preparación de las muestras}

Hemos producido muestras de $\mathrm{Fe}_{\mathrm{x}} \mathrm{Cu}_{100-\mathrm{x}}$ en concentraciones $x=1 ; 5 ; 10 ; 14,1$; $17 ; 18,8 ; 20 ; 25 ; 30 ; 35 ; 40$ y $45 \%$ atómico de hierro. Efectuamos el procedimiento que se indica a continuación: Cargamos el tambor con $1 \mathrm{~g}$ de la mezcla de polvos en las proporciones adecuadas a la estequiometría nominal deseada. Colocamos también 43 bolas de acero de $9 \mathrm{~mm}$ de diámetro. Cerramos el tambor en atmósfera de argón. Con esta carga efectuamos una molienda preliminar de $1 \mathrm{~h}$ a fin de asegurar que las herramientas de molido estaban recubiertas del mismo material a moler. Vaciamos el tambor y les efectuamos un "enjuague" con metanol para retirar el material en polvo suelto. Secamos con aire caliente (a menos de $100{ }^{\circ} \mathrm{C}$ ). Recargamos el tambor con $6 \mathrm{~g}$ de la mezcla original de polvos. La relación de masas entre bolas y polvo fue de 25:1. A fin de evitar la adherencia del material a las bolas y las paredes del tambor, agregamos 0,3 cc. de metanol ${ }^{10}$. Cerramos el tambor en atmósfera de argón. Colocamos el tambor en un molino vibratorio Nissin Giken NEV-MA8 refrigerado por agua. Molimos en tiempos variables para cada una de las concentraciones, a una frecuencia nominal de 13,3 Hz. No retiramos material del cilindro durante la molienda. Los polvos resultantes tienen un color marrón oscuro grisáceo. En ocasiones, al abrir el tambor, el polvo acumulado en los bordes se inflamaba al entrar en contacto con el aire. Medimos el efecto magnetoresistivo para cada preparación.

En este sistema encontramos que el efecto magnetoresistivo disminuye con el tiempo de molido (ligeramente en el caso de las muestras con $x=18,8$ y $20 \%$ ), pero es siempre observable para los tiempos investigados (20 a 150 horas). A fin de evitar incertezas como las producidas por variaciones en la frecuencia de agitación del molino

\footnotetext{
${ }^{10}$ Esto es una práctica usual cuando se muelen materiales dúctiles. Véanse comentarios en el apéndice $A$.
} 
decidimos fabricar las muestras con un tiempo que asegurara que se alcanza un estado estacionario. Por esto la línea principal de investigación sobre este sistema se basa fundamentalmente en las muestras con 75 horas de molido. Donde no se indique el tiempo de molido entenderemos que se trata de estas últimas. 


\section{Medidas}

\subsection{Magnetoresistividad}

En la figura 2 vemos cuatro resultados característicos de las medidas de $\Delta \rho / \rho=\rho(1,4 T)-\rho(0) / \rho(0) \times 100$ tomadas a $77 \mathrm{~K}$, correspondientes a muestras con $x$ $=1,10,17$ y $45 \%$ at. Para la primera no se ve efecto alguno. Para la muestra con $x=10$ $\%$ se esboza una leve respuesta. Para la de $x=17 \%$ el efecto es bien marcado. En el último se ve el efecto muy disminuido. En la figura 3 vemos la evolución del efecto magnetoresistivo con la concentración de hierro para las muestras con 75 horas de molido. Como se ve, el efecto aparece recién en las muestras con $10 \%$ atómico de Fe $(\Delta \rho / \rho=0,5 \%)$, para crecer hasta una concentración de 18,8\% $(\Delta \rho / \rho=2,38 \%)$. Luego
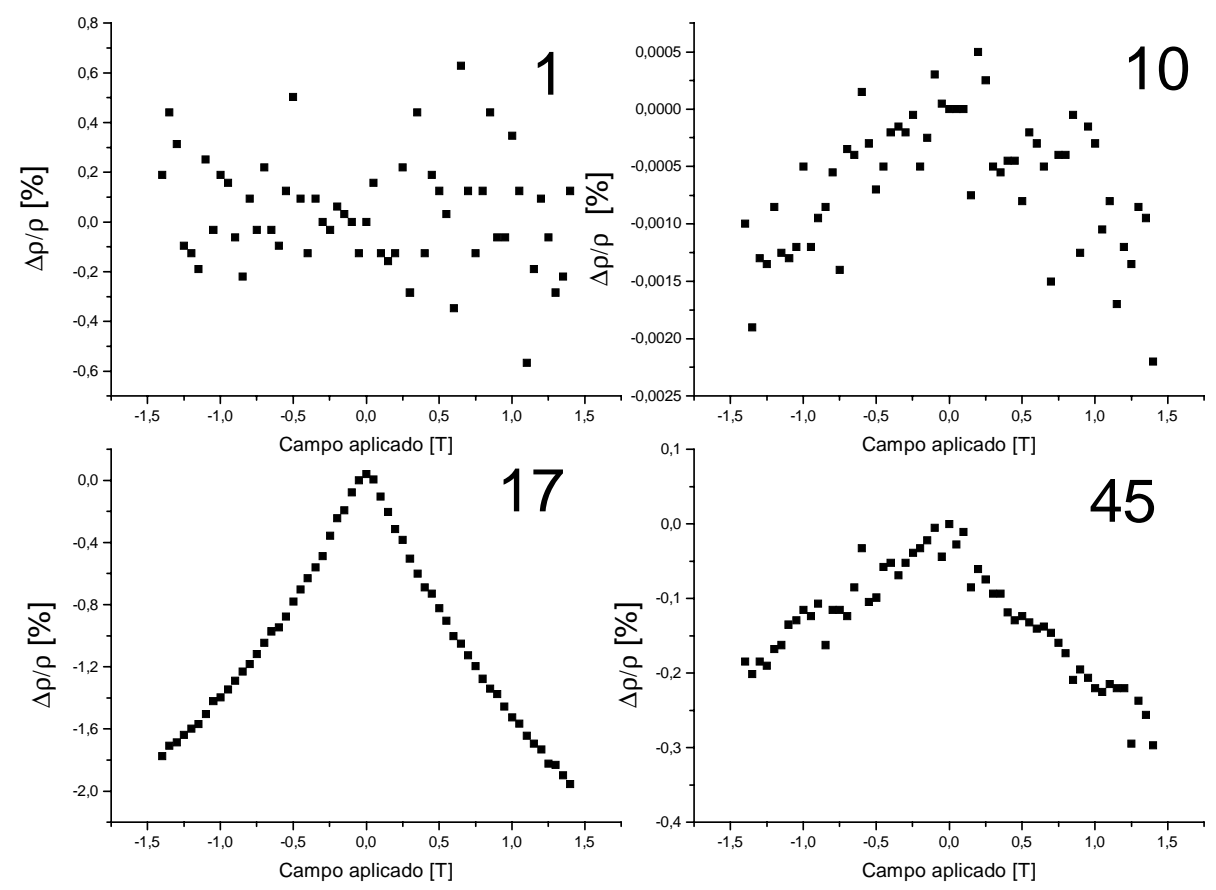

Fig. 2: Magnetoresistividad medida a $77 \mathrm{~K}$ en cuatro muestras de concentraciones diferentes: $1,10,17$ y $45 \%$ at. Fe 
el efecto empieza a disminuir hasta hacerse casi nulo a la máxima concentración investigada, esto es, $45 \%(\Delta \rho / \rho=0,25 \%)$. Aunque estos valores no sean tan notables como los alcanzados en los sistemas multicapas (que pueden ser tan grandes como 150 \% [Levy 1994]), el efecto es isotrópico y negativo. El máximo efecto magnetoresistivo registrado, de $2,65 \%^{11}$, fue para muestras "crudas" de $\mathrm{Fe}_{20} \mathrm{Cu}_{80}$ con 30 horas de molido (fig. 4).

Las medidas tomadas a temperatura ambiente no muestran ningún efecto. Algunas muestras fueron medidas a 4,2 K. El efecto magnetoresistivo medido a esa temperatura fue de 7,4\% para el $\mathrm{Fe}_{18,8} \mathrm{Cu}_{20,2}$ y de $5 \%$ para el $\mathrm{Fe}_{25} \mathrm{Cu}_{75}$, ambas a $1,35 \mathrm{~T}$.

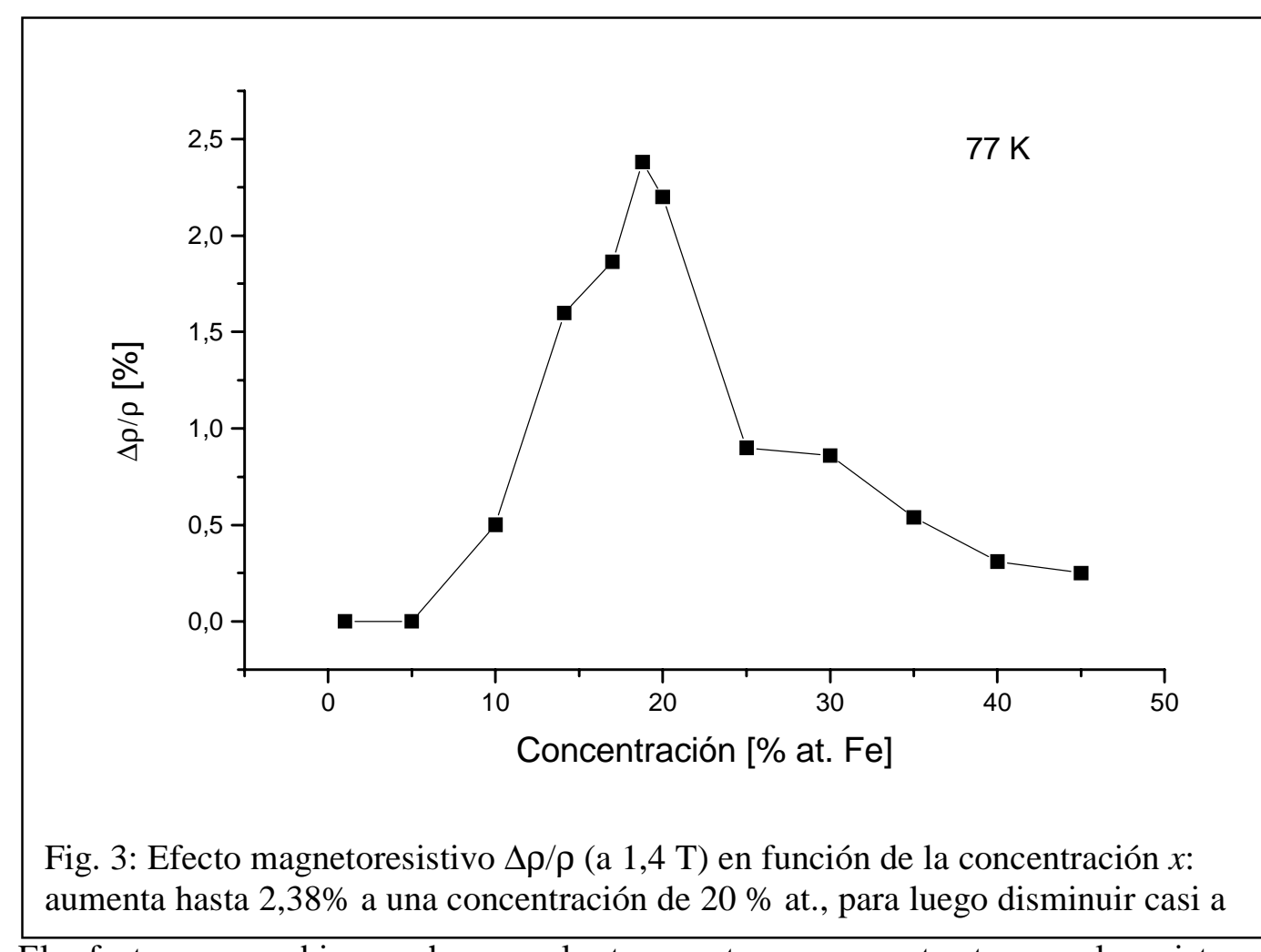

El efecto no cambia mucho con la temperatura, en contraste con los sistemas multicapas, que son fuertemente dependientes de la misma [Levy 1994]. Estos valores no son muy diferentes de los obtenidos por Wang et al. ([Wang 1994] para $x=20 \%$ vol. Fe miden $\Delta \rho / \rho=5,02 \%$ a 4,2 K y campo de 1,4 T), Saxena et al ([Saxena 1994]

${ }^{11}$ Promedio de ambas ramas 
para $x=15$ miden $\Delta \rho / \rho=1,35 \%$ a $77 \mathrm{~K}$ y campo de 1,5 T) y Eilon et al ([Eilon 1995] para $x=25$ miden $\Delta \rho / \rho$ de entre $1,2 \%$ a $1,6 \%$ para temperaturas entre 50 y $300 \mathrm{~K}$, con un campo de 1,4 T); y probablemente menores a los de Ueda et al ([Ueda 1996] para $x=$ 30 miden $\Delta \rho / \rho=0,8 \%$ a temperatura ambiente, con campo de $1,5 \mathrm{~T})^{12}$.

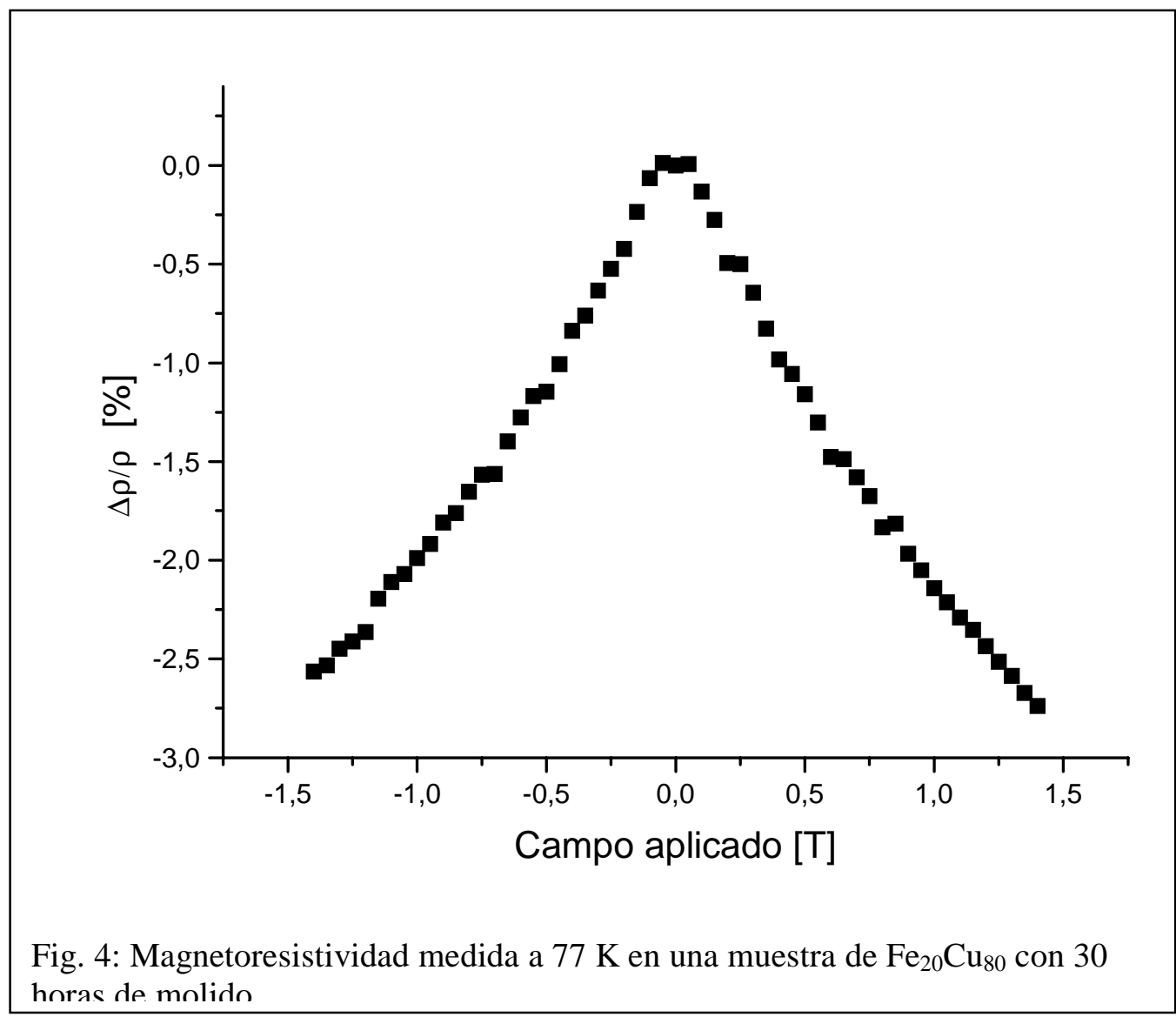

\footnotetext{
${ }^{12}$ Algunos de estos valores fueron estimados de las gráficas presentadas en los trabajos mencionados.
} 


\subsection{Difracción de rayos $X$}

Para la determinación de la estructura cristalina hicimos difracción de rayos $\mathrm{X}$ (DRX) con un aparato Rigaku y otro Phillips PW1170, ambos equipados con lámparas

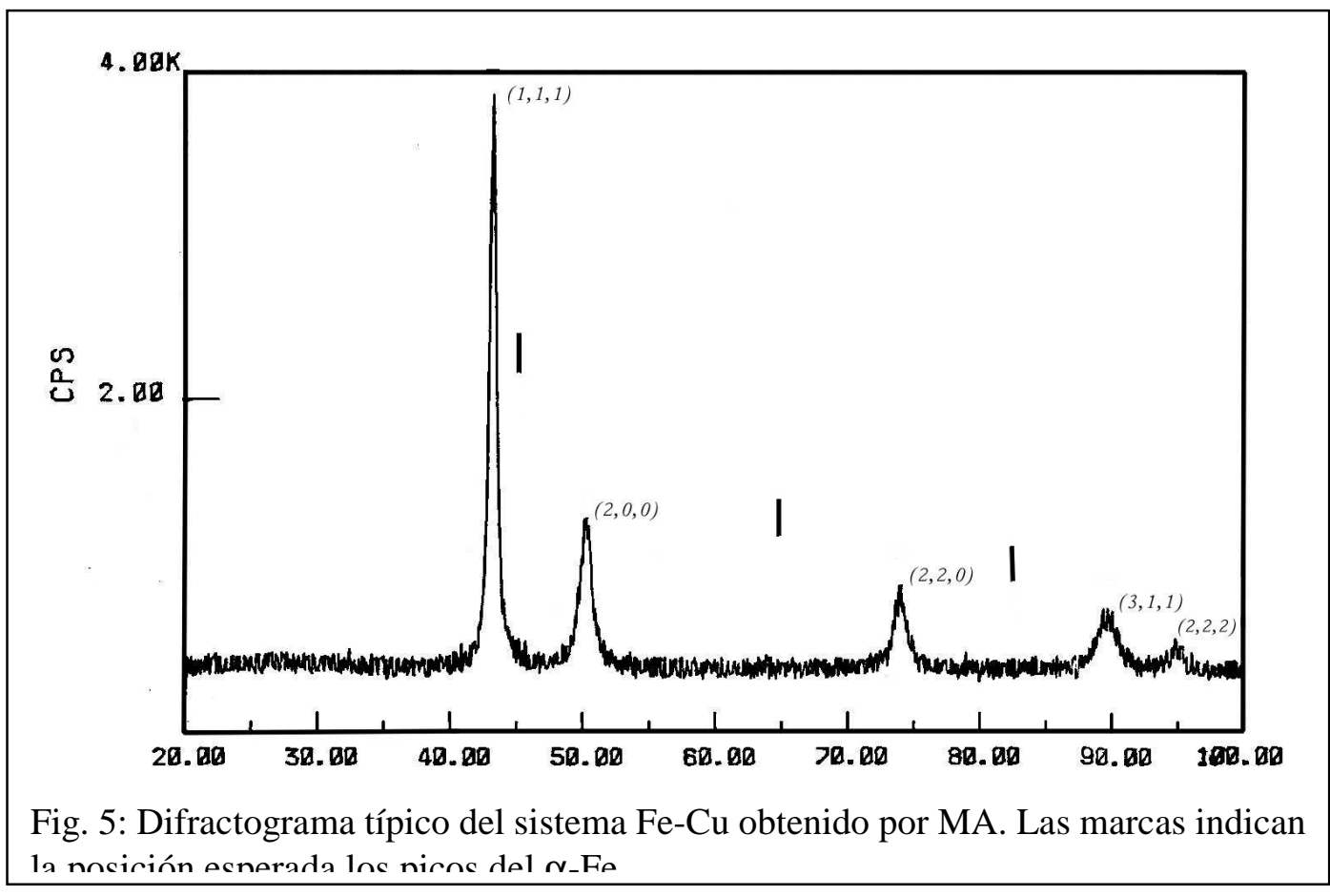

de cobre.

Los difractogramas presentan todos el mismo aspecto del de la figura 5: picos muy próximos a las posiciones del $\mathrm{Cu}$ puro, que es fcc, ensanchados. Calculamos el parámetro de red para cada concentración. El método para obtener el parámetro de red es el siguiente: hemos hecho el cálculo sobre la base de ajustar dos lorentzianas, en la relación 2 a 1 que mantienen $\operatorname{los}$ dos rayos $\mathrm{K}_{\alpha}$ del $\mathrm{Cu}$, a cada uno de los picos distinguibles del espectro; y utilizando que la posición angular $\theta$ de cada pico está relacionada con el espaciado interplanar $d_{h k l}$ por medio de la relación de Bragg: $m \lambda=$ 
$2 d_{h k l} \operatorname{sen} \theta$, donde $m$ es el orden del pico de difracción y $\lambda$ la longitud de la onda emitida por el aparato ${ }^{13}$. Como además $d_{h k l}$ está relacionado con el parámetro de red $a$ por

$$
d_{h k l}=a / \sqrt{h^{2}+k^{2}+l^{2}}
$$

con $h, k$ y $l$ los índices de Miller, entonces graficando $d_{h k l}$ vs. $\left(\sqrt{h^{2}+k^{2}+l^{2}}\right)^{-1}$, la pendiente de esa gráfica nos da el valor medio del parámetro de red sobre todos los picos discernibles. Del análisis

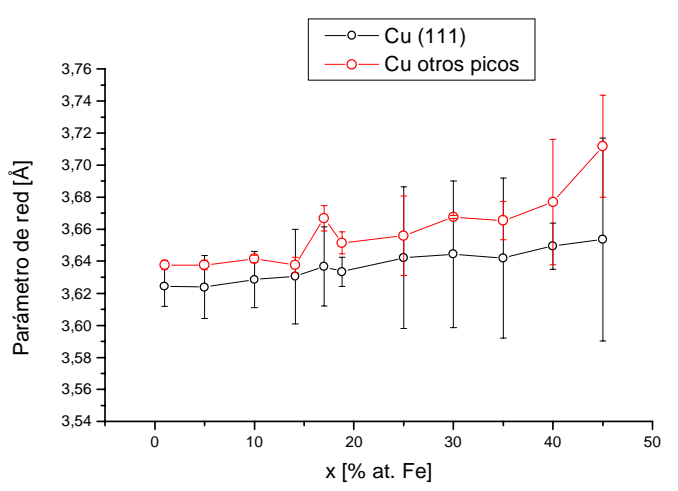

Fig. 6: Parámetro de red calculado con los picos (200), (220) y (311) (cuadrados rojos) y son al nisn (111) (muadrados nasros)

preliminar de estas medidas hemos descartado el pico $(111)^{14}$, ya que tanto su ancho como su posición angular diferían de los otros picos. Sistemáticamente los corrimientos en el espaciado son menores para el pico $(111)^{15}$; así como el ancho del mismo, que se presenta más angostado que el resto. Hemos optado por el cálculo con el resto de los picos, debido a que los puntos en la gráfica propuesta se presentan bastante alineados entre sí, lo que no es el caso del pico (111). De todas maneras, el comportamiento con la concentración es similar para el parámetro de red calculado de ambas maneras ${ }^{16}$ (ver figura 6). Suponemos que el hecho que el pico (111) sea más angosto puede deberse a que hay más planos de red en esa dirección que en las otras, y que el corrimiento del espaciado sea menor puede estar indicando alguna clase de anisotropía, que tal vez se deba a que los cristales estén constreñidos a crecer más en dos dimensiones.

\footnotetext{
${ }^{13}$ Para nuestros cálculos utilizamos $\lambda=1,5406 \AA$, que corresponde al rayo $\mathrm{K}_{\alpha 1}$ y $m=1$. ${ }_{15}^{14}$ Y por ende, el (222).

${ }^{15}$ La diferencia promedio entre ambos parámetros es de 0,2193 nm

${ }^{16}$ Para el parámetro de red calculado con el pico del $\mathrm{Cu}$ (111) hemos utilizado directamente la relación 1.
} 
Como vemos en las figuras 6 y 7 , el parámetro de red aumenta con la concentración de Fe. Los puntos experimentales se encuentran (fig. 7) por encima de lo previsto por la ley de Vegard para una mezcla simple:

$$
a=c a_{F e}+(1-c) a_{C u},
$$

donde $c$ es la fracción atómica relativa y $a_{F e}$ y $a_{C u}$ los parámetros de red del hierro y el cobre respectivamente.

Este resultado ha sido adscripto a efectos de magnetovolumen [Yavari 1992], y no a un problema de tamaños, debido a que los átomos de hierro tienen un volumen similar a los del cobre. Nuestros resultados son ligeramente mayores que los de otros autores, aunque el comportamiento es similar. Que el parámetro de red sea un poco mayor a lo reportado en los otros trabajos puede ser debido a la presencia de carbono, el

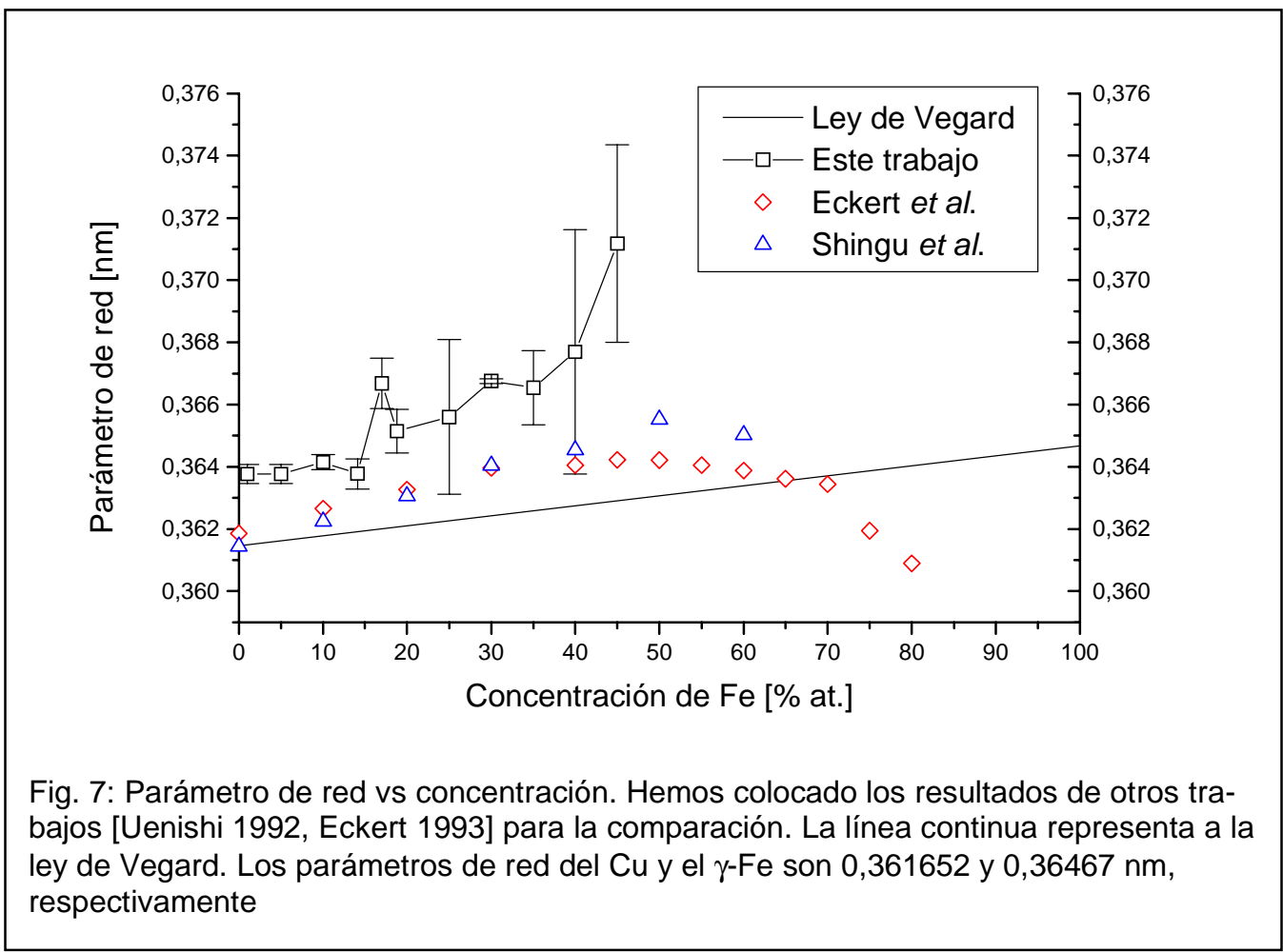


cual se manifestará al hacer el tratamiento térmico de las muestras (ver capítulo II). Este carbono proviene de la descomposición del agente antiadherente utilizado ${ }^{17}$. No hay evidencia de la formación de carburos en los difractogramas de las muestras molidas (eso ocurrirá cuando se haga el tratamiento térmico). También es posible que en los otros trabajos el parámetro de red haya sido calculado utilizando exclusivamente el pico

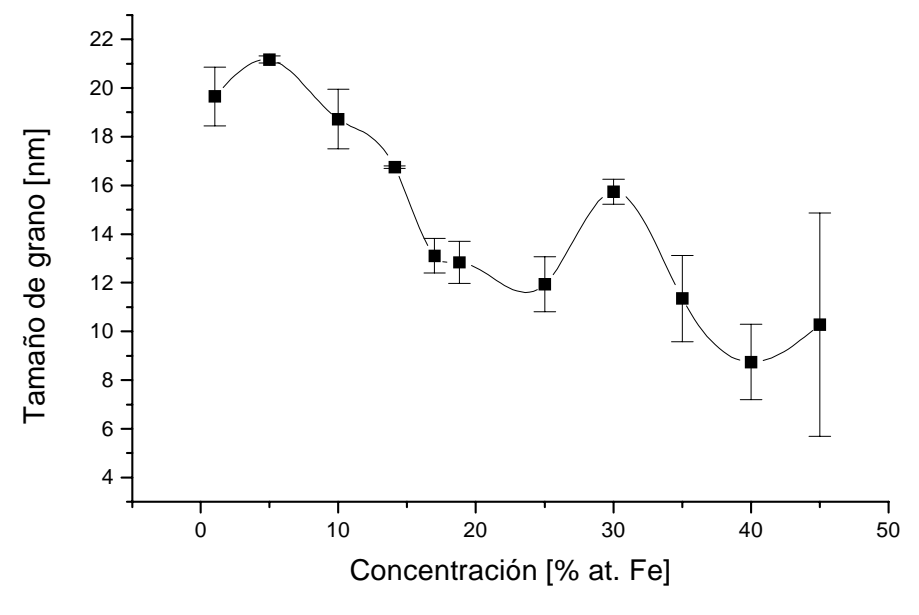

Fig. 8: Tamaño de grano vs. concentración. El tamaño medio de los granos para todas las concentraciones está dentro del ranon $87-711 \mathrm{~nm}$

(111), con lo cual el acuerdo es muy bueno ${ }^{18}$.

\section{Tamaño de grano:}

Para el cálculo de los tamaños de grano utilizamos el conocido método del ancho integrado $^{19}$. De allí obtuvimos que el tamaño medio de los granos de la aleación está en un rango de 8,7 a 21,1 nm, como se ve en la figura 8 . También se ve que hay una caída en el tamaño con la concentración. Las tensiones varían, con un máximo de 0,3 \% para

${ }_{17}^{17} \mathrm{CH}_{3} \mathrm{OH}$ (metanol)

${ }^{18}$ No es el caso del parámetro de red calculado en [Uenishi 1992], donde toman el pico (220) para ese cálculo.

${ }^{19}$ [Klug 1974], página 661 
la muestra con $40 \%$ de Fe. Estos valores concuerdan con los de la literatura ${ }^{20}$ [Eckert 1993].

\subsection{Resistividad}

Medimos la resistividad a $77 \mathrm{~K}$, también con el método de las cuatro puntas. Como vemos en la figura 9, la resistividad aumenta con la concentración de hierro, según la regla de Nordheim. Ésta es una ley empírica que sigue la resistividad de una mezcla de soluto A de concentración $x$ con un solvente B de concentración $100-x$ :

$$
\rho \propto x(100-x)
$$

Esta regla es bastante general, aunque se aplica a las mezclas de metales de transición con metales nobles con la salvedad que pueden aparecer anomalías. Estas anomalías se reflejan como desviaciones de la regla. Estamos interesados en ver el comportamiento "grueso" del sistema, y vemos que la regla aplica bastante bien, con lo

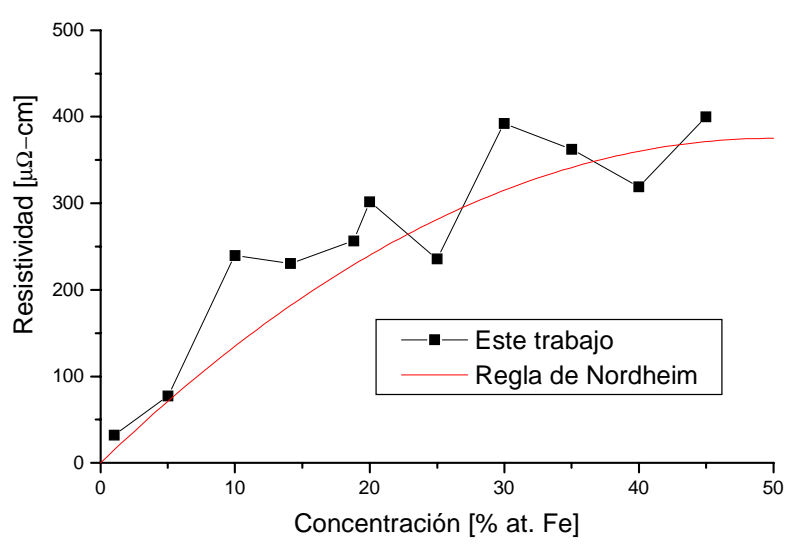

Fig. 9: Resistividad en función de la concentración,

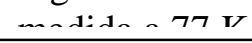
que podemos concluir que nuestras aleaciones constan de "impurezas" o entes dispersores dispuestos aleatoriamente en la matriz provista por el elemento mayoritario.

\subsection{Medidas de susceptibilidad magnética}

\footnotetext{
${ }^{20}$ En esa referencia, para la muestra $\mathrm{Fe}_{30} \mathrm{Cu}_{70}$, el tamaño de grano reportado es $\approx 13 \mathrm{~nm}$ y la tensión media cuadrática $\approx 0,3 \%$
} 

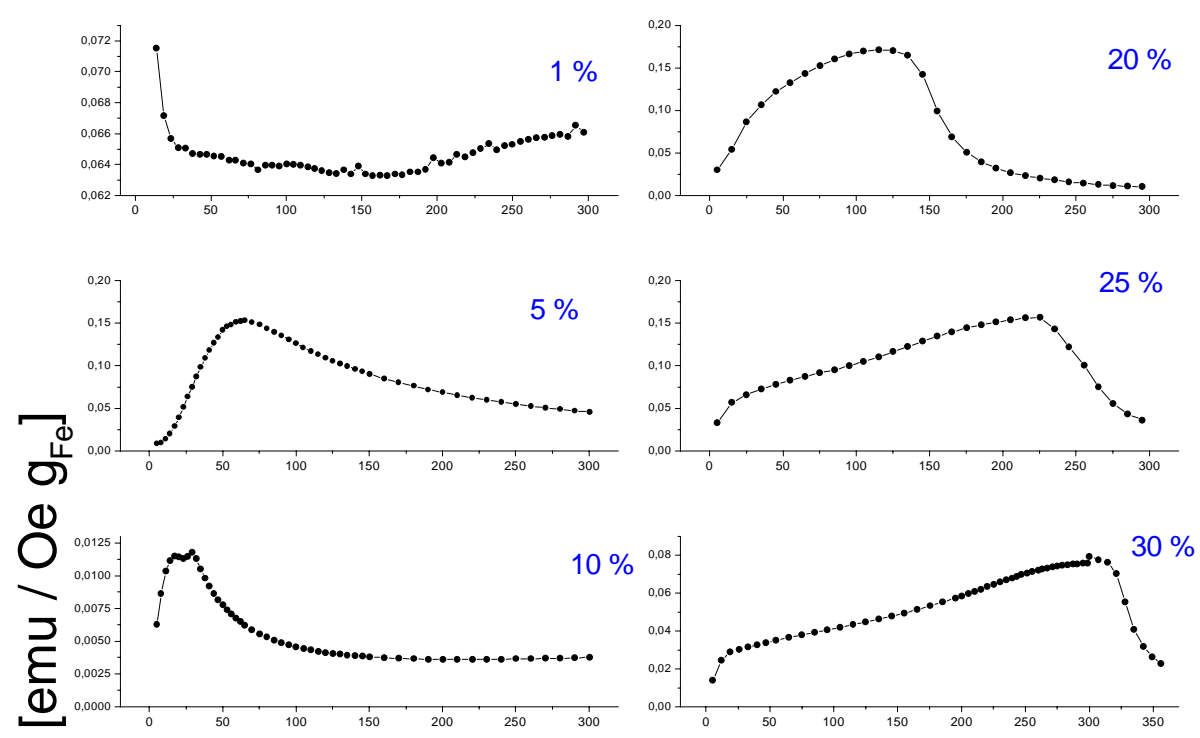

$\times$
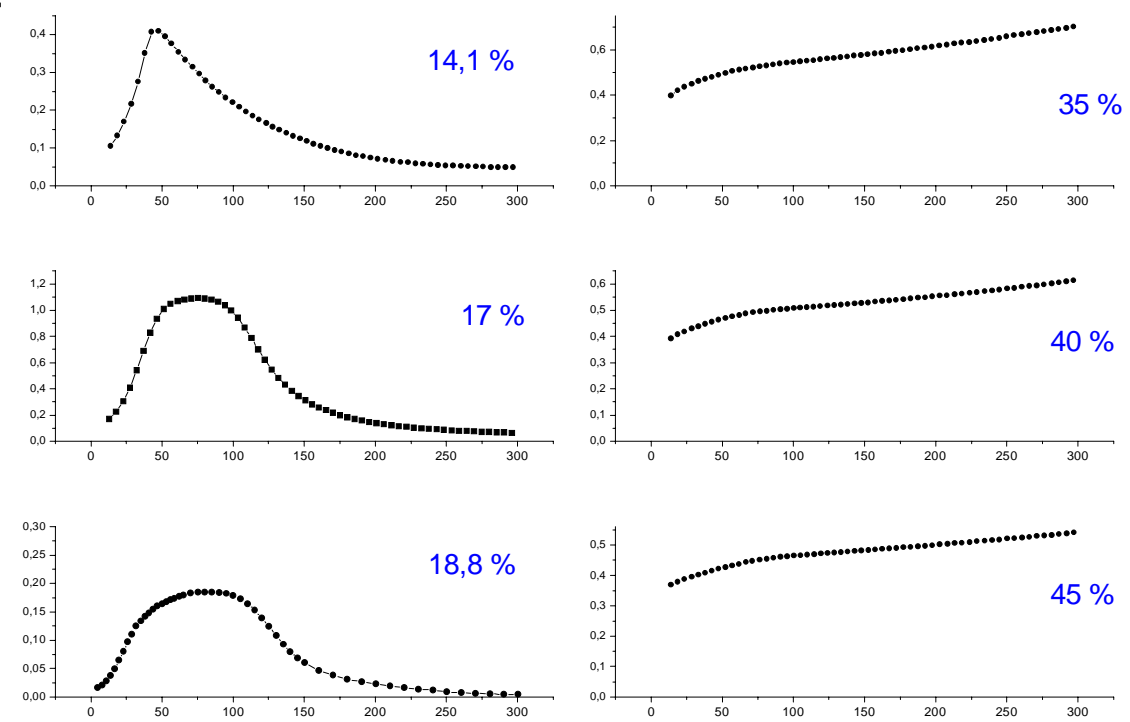

Temperatura $[\mathrm{K}]$

Fig. 10: Medidas de susceptibilidad estática $(H=20 \mathrm{Oe})$ y dinámica $(\omega=375 \mathrm{~Hz}$ y $H=1 \mathrm{Oe}$ ) de las distintas muestras. Ambas fueron tomadas luego de haber sido enfriadas cin camno anlicadn (7Fr)

Hemos efectuado medidas de magnetización vs. temperatura de algunas muestras con un SQUID (Dispositivo superconductor de interferencia cuántica) ${ }^{21}$ marca Quantum Design a bajo campo (20 Oe); y de susceptibilidad de las otras muestras con

${ }^{21}$ Ver apéndice C. 
un susceptómetro marca LakeShore 7130 (con campos oscilantes aplicados de 1 y 10 Oe de amplitud RMS). Con el primero hemos obtenido la susceptibilidad estática $M / H$ de algunas muestras, y con el segundo la susceptibilidad dinámica $\partial M / \partial H$. Como vemos, las medidas son conceptualmente diferentes, pero para las zonas donde $\mathrm{M}$ varía suavemente con $\mathrm{H}$ son aproximadamente iguales. En la figura 10 vemos las medidas de susceptibilidad estática y dinámica tomadas. La muestra con $x=1 \%$ presenta una caída en la susceptibilidad desde $13 \mathrm{~K}$, que es la temperatura mínima alcanzable por el susceptómetro. Las muestras con $x=5,14,1,17$ y 18,8 \% muestran un comportamiento bastante similar a bajas temperaturas, con una caída hacia $\mathrm{T}=0 \mathrm{~K}$ cóncava hacia arriba. La susceptibilidad para $x=20 \%$ no tiene un comportamiento definido, y parece ser un estado transicional hacia las muestras de concentraciones mayores. A $x=25 \%$ vemos que el comportamiento es diferente: si extrapolamos a $\mathrm{T}=$ $0 \mathrm{~K}$, la magnetización no es nula (la pendiente disminuye con $\mathrm{T}$ en la primera parte, y vemos un "hombro" a T $<50 \mathrm{~K}$, lo que se conoce como reentrancia). En las muestras con $x=35,40$ y $45 \%$ ya no vemos el máximo en la susceptibilidad, pero sí es reconocible el hombro o reentrancia de la parte inferior, similar a la de $x=25$ y $30 \%$. Este comportamiento había sido observado en el sistema Fe-Au por Coles, Sarkissian y Taylor en 1978 [Coles 1978], y explicado en detalle en 1981 [Sarkissian 1981]. Según estos autores, el sistema se comporta así debido a que existen aglomeraciones de átomos de hierro, llamados "clusters", los que poseen un espín neto de momento magnético grande. Estos clusters hacen que en el sistema coexistan dos interacciones magnéticas: la ferromagnética de los clusters y la $\mathrm{RKKY}^{22}$. A bajas concentraciones de soluto

\footnotetext{
${ }^{22}$ Por las iniciales de Ruderman, Kittel, Kasuya y Yoshida. Ellos establecieron que para espines aislados unos de otros, existe una interacción efectiva modulada de largo alcance, que puede escribirse como $I_{R K K Y}=\frac{A(r)}{r^{3}} \cos \left[2 k_{F} r+\delta(r)\right]$.
} 
magnético (pero no tan bajas como para que tengamos un 'vidrio de espines' o spinglass canónico), el sistema consiste en una dispersión de estos clusters en un medio no magnético $^{23}$. Si el sistema fue enfriado a T próximo a $0 \mathrm{~K}$, los momentos magnéticos de los clusters quedan orientados al azar, mediados por la interacción RKKY. Esto da como resultado que la magnetización a temperatura muy baja sea prácticamente cero. Al calentar el sistema, los clusters pierden progresivamente la orientación en la que habían quedado al congelarse (generado por la RKKY). Por tanto, se van viendo libres de seguir al campo aplicado y de esta manera pierden la coordinación con los otros clusters. Esto ocurre hasta un punto donde la agitación térmica hace que el momento magnético de cada cluster pierda la orientación con el campo externo. A partir de entonces, nuestra técnica de detección no puede registrar la magnetización de esos clusters, con lo que la magnetización neta baja, y eso es lo que vemos en nuestros gráficos. La caída no es abrupta, y eso puede deberse a que los tamaños de los clusters están distribuidos, con lo que los clusters más pequeños son quienes comenzarán a convertirse en superparamagnéticos ${ }^{24}$ antes. Con el posterior

${ }^{23}$ Esta clase de sistemas es conocido como "cluster-glass" o mictomagnético. El prefijo "micto" proviene del griego y significa "mezclado".

${ }^{24}$ Entendemos que para una partícula pequeña existen ejes de facil magnetización [Mørup 1980], característicos de cada sistema. La energía anisotrópica es $E_{a}=K V$, donde $K$ es la constante de anisotropia y $V$ el volumen de la partícula. Cuando la energía térmica es del orden de esta energía, la magnetización de la partícula (en nuestro caso, el cluster) "salta" entre los ejes de fácil magnetización. En cada posición permanece un tiempo $\tau$, definido como $\tau=\tau_{0} e^{\Delta E / k_{B} T}$ donde $\tau_{0}$ es una constante del orden de $10^{-9}-10^{-11} \mathrm{~s}$, y $\Delta E=K V \operatorname{sen}^{2} \theta-H M \cos (\alpha-\theta)$, con $\theta$ el ángulo subtendido entre el eje de fácil magnetización y el momento magnético, y $\alpha$ el ángulo entre el eje y el campo aplicado (suponiendo la existencia de un solo eje fácil). En esta situación, cuando queremos medir la 
aumento de la temperatura todos los clusters "pierden" (en el sentido de que no podemos medir) su momento magnético. Cuando la concentración de soluto magnético pasa de un cierto límite (el llamado "umbral de percolación", $\left.\mathrm{C}_{\mathrm{p}}\right)^{25}$ los clusters empiezan a tocarse unos con otros (ver figura 10), lo que produce que entre éstos la interacción dominante ya no sea la RKKY sino dipolar o de intercambio, o la coexistencia de ambas en la muestra. Como esto ocurre progresivamente con el aumento de la concentración, cercano a $\mathrm{C}_{\mathrm{p}}$ tendremos muchos clusters todavía aislados, que interactúan entre sí vía la RKKY. Pero el orden ferromagnético se va haciendo dominante en la medida en que más y más clusters percolan. Para el sistema percolado es esperable un comportamiento como el visto en las

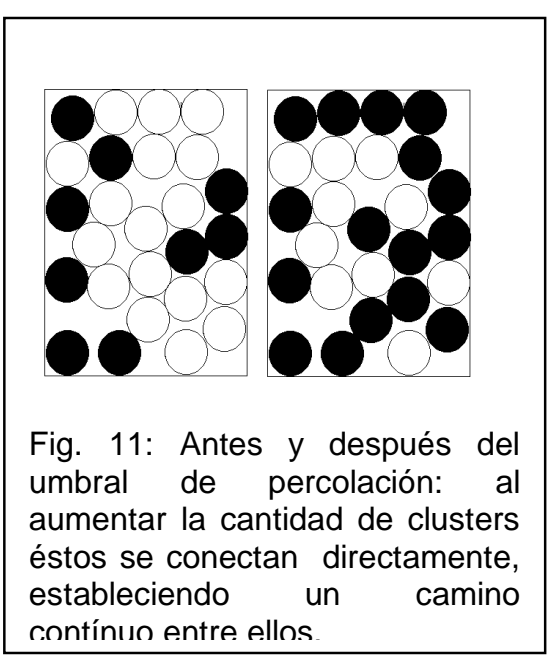
concentraciones $x \geq 25 \%$ at.: la magnetización es no nula para $\mathrm{T} \approx 0 \mathrm{~K}$, lo que ocurre debido a que el o los clusters percolados tienen una magnetización remanente (que es lo que se conoce como reentrancia). Al "descongelarse", los clusters libres se orientan con

magnetización de nuestro sistema, si nuestra técnica de medida tiene un tiempo característico mayor a $\tau$, vamos a estar promediando la magnetización en ese tiempo. Como esta magnetización es proporcional al $\operatorname{coseno}(\theta)$, al promediar compensamos la magnetización en una orientación con la de la orientación opuesta. El resultado es que la magnetización promedio es nula. Este fenómeno es conocido como superparamagnetismo (SPM). Esta descripción no tiene en cuenta la interacción con otras partículas, como es posible que tengamos en nuestro sistema.

${ }^{25}$ La noción de percolación [Essam 1980] es, en principio, geométrica: se dice que, dados dos grupos de entes que forman un sistema, éste percola cuando es posible establecer un camino que conecte a todos los entes de una clase. En nuestro caso, entendemos por umbral de percolación a la concentración donde un grupo de clusters se conecta directamente, formando así un cluster macroscópico. Este cluster es susceptible de partirse en dominios, como un ferromagneto macroscópico. Con el aumento de la concentración aparecen más clusters macroscópicos, y así siguiendo. Entenderemos como percolación completa a la concentración donde no quedan clusters aislados. Nuestro caso no es estrictamente un problema de percolación de sitio, ya que dos clusters suficientemente próximos aunque no en contacto directo tendrán una interacción adicional (p.e. dipolar), por lo que entenderemos que en nuestros sistemas tenemos percolación magnética. Y así el umbral de percolación estará dado por la concentración donde cambia el carácter de la curva de susceptibilidad. Esto es dificultoso de determinar, como se comenta en el texto principal. 
el campo. La respuesta al campo aplicado crece en la medida que la temperatura aumenta, al verse libres los clusters de las interacciones con sus vecinos. La susceptibilidad se incrementa notablemente, como lo hace un ferromagneto, hasta alcanzar un máximo. La longitud de correlación entre los espines aumenta, hasta hacerse muy grande comparada con el tamaño de los clusters originales (esa es la razón del pico aguzado que aparece en la medida). Luego, las fluctuaciones térmicas se hacen tan grandes que hacen que el sistema vaya dejando de seguir al campo aplicado, como vemos de la caída de la susceptibilidad con T. El orden ferromagnético descripto no lo es en el sentido de un ferromagneto canónico, donde toda la muestra tiene ese carácter. Lo que significamos es que las interacciones dominantes son la de intercambio y/o dipolar. Una medida de la interacción entre las partículas está dado por el cálculo de la temperatura de Weiss $\Theta$ a partir de la medida de la susceptibilidad. Como la ley de Curie Weiss establece que :

$$
\chi=\frac{C}{T-\Theta}
$$

entonces, graficando la inversa de la susceptibilidad vs $\mathrm{T}$ y ajustando a la zona lineal (correspondiente a las temperaturas altas) una recta, es posible determinar dicho valor. En la figura 12 vemos la temperatura de Weiss en función de la concentración de hierro, y vemos que para las concentraciones más bajas

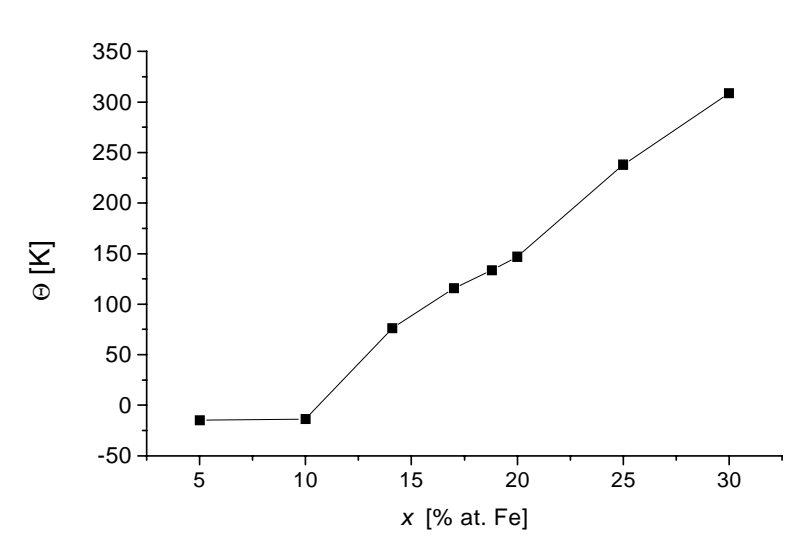

Fig. 12: Temperatura de Curie $\Theta$ vs concentración. No fue posible calcular esta temperatura para $x>30$ $\%$, debido a que sus temperaturas críticas están por encima del alcance de nuestro succentómetro 
es ligeramente negativa. Luego aumenta linealmente con la concentración. Este comportamiento nos dice que el orden predominante entre clusters es antiferromagnético para $x \leq 10$, y luego ferromagnético, con una intensidad creciente con la concentración.

Hemos supuesto que al pasar la temperatura crítica, el único efecto magnético que teníamos era el de perder la magnetización macroscópica, pero alternativamente podemos pensar en que cada cluster hace, a la temperatura crítica, una transición a un estado no magnético (o sea, que la interacción de intercambio entre cada átomo de cada cluster se ve destruida por la temperatura). No es posible sólo con esta técnica determinar si lo que ocurre es lo uno o lo otro. Sería posible ver esto haciendo una medida con una técnica de tiempo característico corto (P.E. espectroscopía Mössbauer), a una temperatura por encima de $\mathrm{T}_{\mathrm{c}}$, y con campo aplicado. Esta segunda causa parece improbable, ya que la interacción dominante dentro de un cluster puede ser ferromagnética, con lo que la temperatura de Curie sería más alta que las temperaturas medidas. En la Discusión volveremos sobre este punto.

Calculamos las temperaturas críticas a partir de la interpretación dada ${ }^{26}$, y confeccionamos un diagrama de fases magnéticas (figura 13). Para las muestras con $\mathrm{x}<$ $18,8 \%$ tomamos la temperatura donde la derivada primera de la susceptibilidad se anula $^{27}$ para medir la llamada "temperatura de vitrificación"28. Para las concentraciones mayores tomamos dos temperaturas características, la del "hombro" y la de cambio entre el comportamiento ferromagnético (FM) y el paramagnético (PM). A la primera la definimos como la intersección entre las rectas que siguen a la parte superior del

\footnotetext{
${ }^{26}$ En las medidas obtenidas con el susceptómetro hemos promediado los resultados obtenidos para cada frecuencia utilizada.

${ }^{27}$ Lo definimos así para evitar la incerteza que puede aparecer en los picos redondeados, como en $\mathrm{Fe}_{17} \mathrm{Cu}_{83}$

${ }^{28}$ Del inglés "glassy temperature", aunque deberíamos hablar de una temperatura de "desvitrificación".
} 


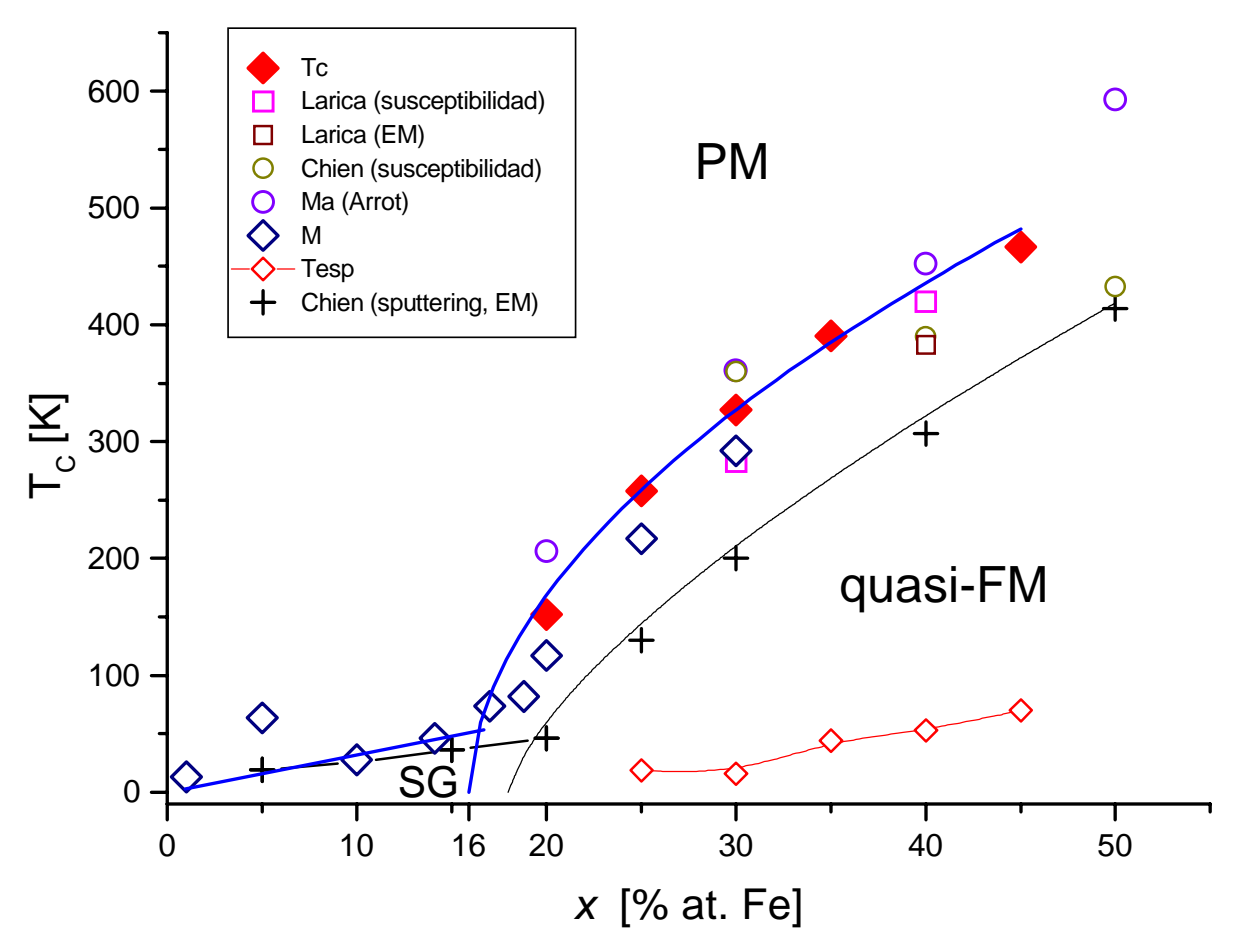

Fig. 13: Diagrama de fases magnéticas para el $\mathrm{Fe}-\mathrm{Cu}$ preparado por aleamiento mecánico. PM, quasi-FM y SG indican las zonas de comportamiento paramagnético, cuasi-ferromagnético y spin-glass, respectivamente. Incluimos las temperaturas obtenidas por los grupos de Ma [Ma 1993], Larica [Monteiro 1998] y Chien [Ambrose 1993]; y a título de comparación, muestras producidas por sputtering [Chien 1986]. EM significa que las temperaturas fueron obtenidas por espectroscopía Móssbauer. Ma las obtuvo utilizando gráficos de Arrot. Nuestras temperaturas (diamantes rojos llenos) fueron obtenidas como describimos en el texto. Añadimos las temperaturas de los máximos ( $\mathrm{M}$, diamantes abiertos azul marino) para comparación. Las temperaturas para $x=35$ y 45 fueron obtenidas por

"hombro" (la "meseta") y a la "subida" 29 . Podemos mencionar que aproximadamente a

esta temperatura se registra un máximo en la componente fuera de fase de la susceptibilidad dinámica. La segunda de estas temperaturas es la que marca el cambio en el comportamiento global del sistema. A esta temperatura la llamaremos "temperatura crítica", si bien no sabemos si se trata de una auténtica transición FM-PM (en cuyo caso sería la temperatura de Curie), o es el paso al estado superparamagnético (en cuyo caso sería la temperatura de bloqueo). Definimos la temperatura crítica $\left(\mathrm{T}_{\mathrm{C}}\right)$

\footnotetext{
${ }^{29}$ Esta definición es arbitraria, pero como la existencia de un comportamiento crítico es todavía un punto controversial, nos inclinamos a hacerlo al estilo de C. L. Chien [Childress 1991].
} 
como la temperatura a la que se anula la derivada segunda de la curva ${ }^{30}$. Como podemos apreciar, las temperaturas críticas obtenidas siguen la tendencia de aquellas medidas obtenidas por espectroscopía Mössbauer en sistemas preparados por sputtering ([Chien 1986]). Las temperaturas reportadas por Ma et al. fueron obtenidas con gráficos de Arrot. La temperatura obtenida por el grupo de Larica es la temperatura del máximo en la susceptibilidad. Como vemos, existen ambigüedades en la definición de $\mathrm{T}_{\mathrm{C}}$ que hacen que las temperaturas reportadas difieran. Es un hecho también conocido que las temperaturas críticas cambian según la técnica de medida, no solamente debido a un posible comportamiento superparamagnético, sino a que hay técnicas más sensibles que otras al orden de corto alcance. Una medida Mössbauer dará una temperatura de orden mayor que la de una medida de magnetización ([Mørup 1994]), y éste es un hecho experimental ([Mørup 1994, Monteiro 1998]).

La línea crítica o de cambio de fase está por encima de la de las muestras obtenidas por sputtering, lo que está indicando, en principio, una mayor homogeneidad de estas últimas. Esta homogeneidad debemos entenderla como que la composición es constante a lo largo de toda la muestra. La línea que une nuestras $\mathrm{T}_{\mathrm{C}}$ fue utilizada, al igual que en [Sarkissian 1981] y [Chien 1986], para calcular el umbral de percolación $\mathrm{C}_{\mathrm{p}}$ mediante la expresión:

$T_{c} \alpha\left(x-C_{p}\right)^{\gamma}$,

donde tomamos sólo las temperaturas críticas de las curvas percoladas (y la de $20 \%$, quien no tiene un comportamiento definido) y que resultó en $\mathrm{C}_{\mathrm{p}}=15,9 \pm 3,2 \%$ atómico. Esta concentración es levemente menor al obtenido por Chien $\left(\mathrm{C}_{\mathrm{p}}=18,5 \%\right)$, pero dentro

\footnotetext{
${ }^{30}$ Lo definimos así debido a la dispersión de tamaños de partículas, con posibles diferencias composicionales entre sí. Esto haría que para cada cluster haya una temperatura de Curie. Como el cambio en la curvatura es manifestación de que un comportamiento se ha impuesto sobre el otro (en este caso el ferromagnético y el paramagnético o superparamagnético), nos ha parecido adecuada la definición. Otros autores han hecho uso de la misma definición [Childress 1991].
} 
de lo previsto en [Mydosh 1993] para una percolación magnética de carácter FM (17 $\%)$.

Si bien en algunos trabajos se utiliza la palabra "de Curie" para referirse a las temperaturas críticas (nuestras $\mathrm{T}_{\mathrm{C}}$ ), seguimos entendiendo que lo que ocurre al pasar la línea crítica es un cambio en el comportamiento magnético macroscópico, donde los momentos magnéticos ya no siguen al campo aplicado debido a las fluctuaciones térmicas. Si interpretamos que tenemos una colección de clusters superparamagnéticos, nuestra temperatura crítica es la temperatura promedio de bloqueo superparamagnético. Estaría expresando que la mitad de los clusters tiene oscilando sus espines a tiempos característicos menores a nuestro tiempo de medida, si se tratase de clusters que no interactúan entre sí. Como la parte "descendente" (para T por encima de la temperatura crítica) de una curva de susceptibilidad ZFC sería describible con una función de Langevin:

$$
M=M_{S} L\left(\mu H / k_{B} T\right)
$$

con $M_{S}$ la magnetización de saturación, $L$ la función de Langevin, $\mu$ el momento, $H$ el campo aplicado y $k_{\mathrm{B}}$ la constante de Boltzmann. Para una distribución de tamaños y momentos tendremos una distribución de funciones de Langevin. Entonces, esa parte de la curva ZFC (también llamada "reversible") nos está describiendo la distribución de tamaños. Los intentos por ajustar con funciones de Langevin simples a las medidas originales de magnetización versus $T$ no fueron exitosos. Probablemente esto se deba a que el sistema está formado por partículas fuertemente interactuantes.

Un criterio que puede ayudar a dilucidar si tenemos un mictomagneto o un superparamagneto es el cálculo de una relación entre el cambio en las temperaturas críticas halladas en las medidas de susceptibilidad a distintas frecuencias (propuesto por 
Mydosh ${ }^{31}$ ). Hemos encontrado que $W$ no supera 0,027 , lo que indica que el comportamiento de nuestras muestras es similar al de un mictomagneto, más que de un superparamagneto clásico (sin interacción).

${ }^{31}$ Ver §III.5 


\subsection{Medidas Mössbauer}

La espectroscopía Mössbauer (EM) es una técnica resonante nuclear que permite la medición de algunos parámetros hiperfinos de un sistema que contenga el isótopo apropiado.

Hemos efectuado medidas por EM, utilizando la geometría convencional de transmisión, con una fuente de ${ }^{57} \mathrm{Co}-R h$, en modo de aceleración constante y a

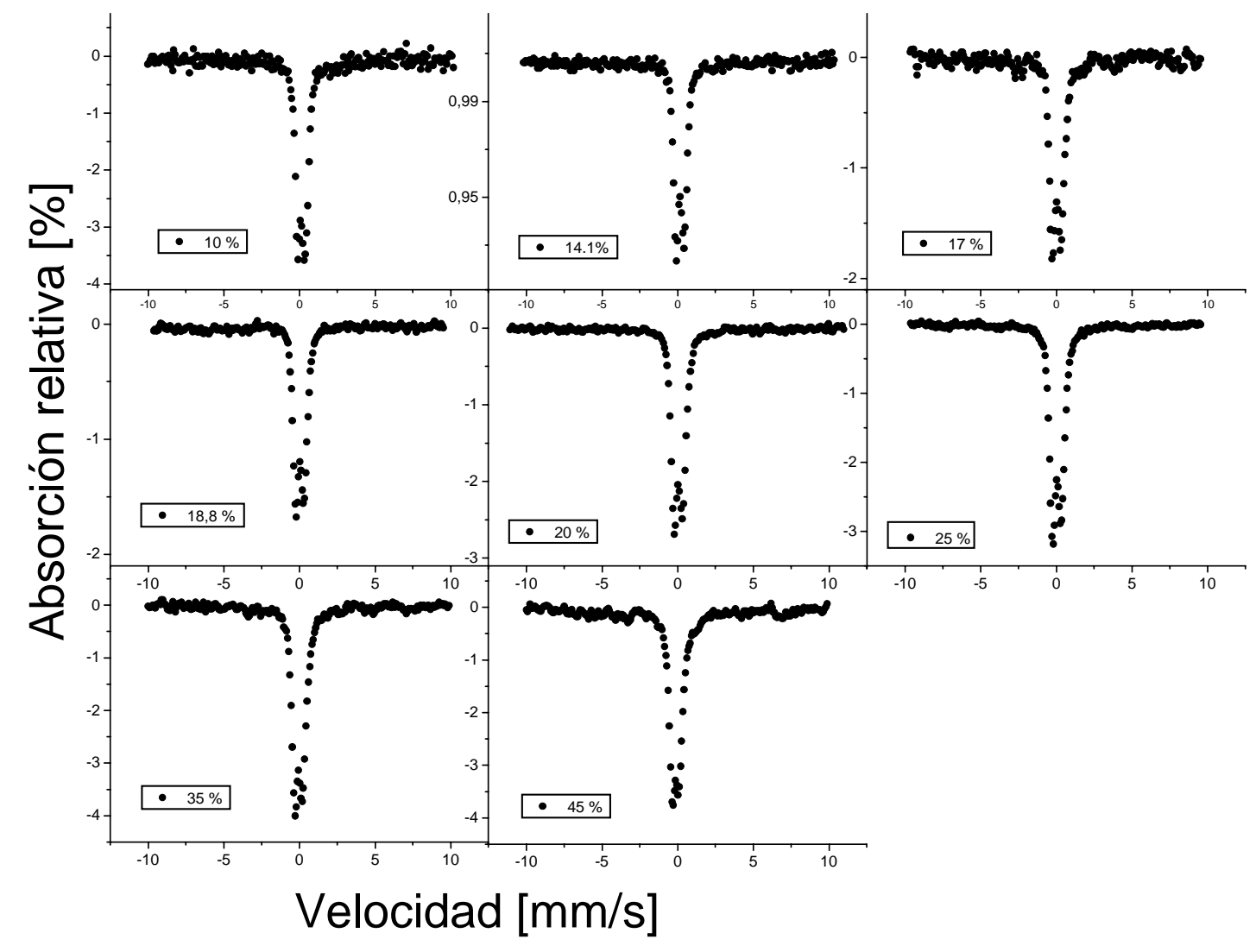

Fig. 14: Arriba: espectros Mössbauer de seis de las muestras de concentraciones menores, tomados a temperatura ambiente. Abajo: espectros de $\mathrm{Fe}_{35} \mathrm{Cu}_{65}$ y $\mathrm{Fe}_{45} \mathrm{Cu}_{55}$ tomados a $407 \mathrm{~K}$ y a $502 \mathrm{~K}$, respectivamente. Indicamos las concentraciones en cada caso.

temperatura ambiente. Se hicieron algunas medidas a bajas temperaturas en el laboratorio del Prof. Nasu ${ }^{32}$ y en el de la Prof. Saragovi ${ }^{33}$. En dos casos (para $x=35$ y

${ }^{32}$ Departament of Material Physics, Universidad de Osaka, Japón 
$45 \%)$ hemos efectuado medidas a altas temperaturas con un horno Austin Science modificado. Para estas dos muestras efectuamos una medida Mössbauer común, a una temperatura suficientemente alta (por encima de lo que anticipaba nuestra línea de cambio de fase en el diagrama $T-x$ del parágrafo $§ I .3 .4)$ que en ambos casos está por debajo de la temperaturas mínimas de precipitación de fases reportadas en varios trabajos $\left(250{ }^{\circ} \mathrm{C}\right)$. Tomando cada medida de alta temperatura como referencia, medimos

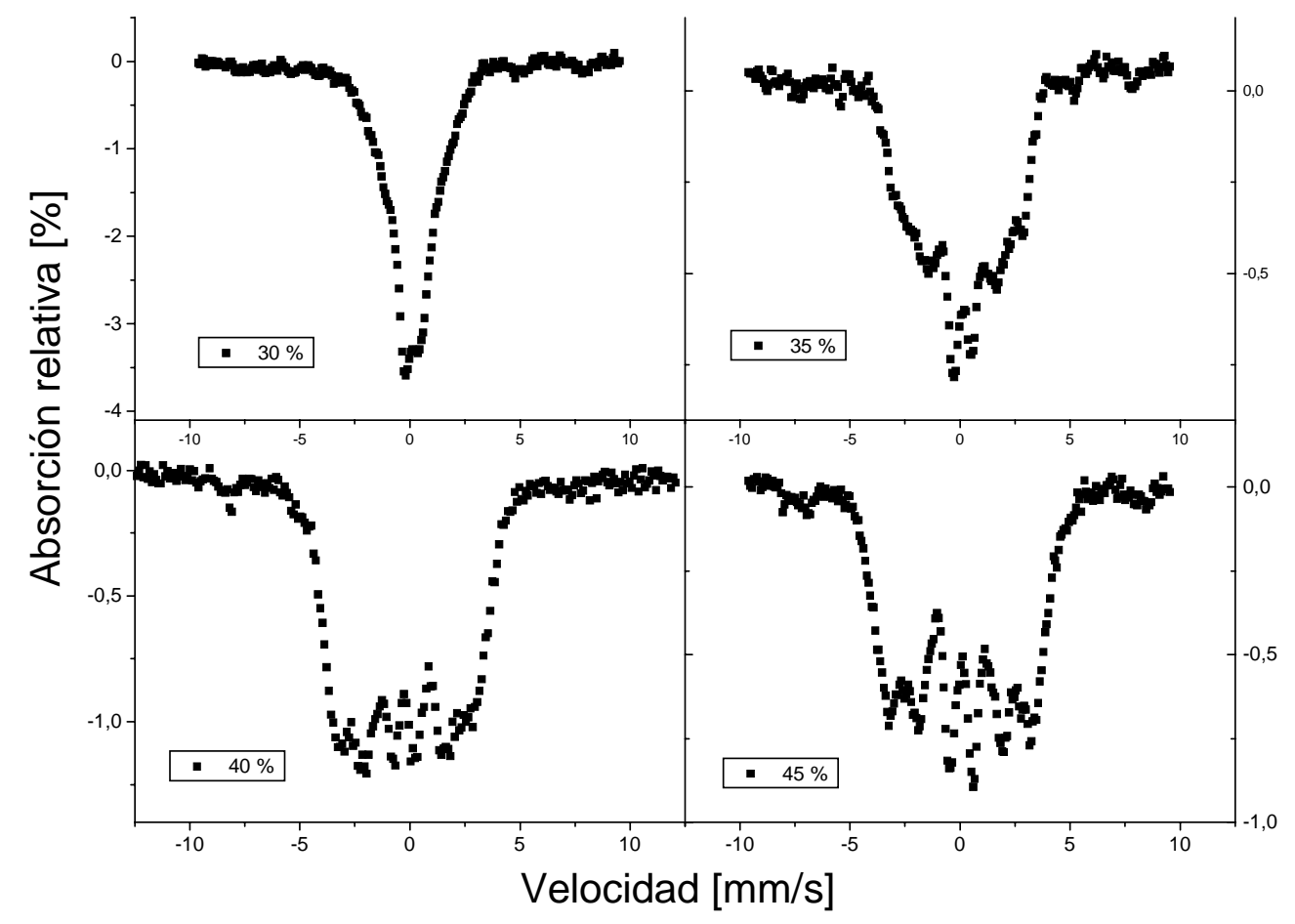

Fig. 15: Espectros Mössbauer de la muestras de concentraciones mayores, tomados a temperatura ambiente. Indicamos las concentraciones en cada caso.

la absorción a una velocidad fija, haciendo barridos en temperatura.

En las figuras 14 y 15 podemos ver la evolución de los espectros Mössbauer con la concentración. A temperatura ambiente los espectros de concentraciones $x=10$; 14,$1 ; 17 ; 18,8 ; 20$ y $25 \%$ at. muestran solamente un doblete ensanchado ligeramente asimétrico. El espectro para $x=30 \%$ at. es todavía un doblete, pero el ensanchamiento

${ }^{33}$ Centro Atómico Constituyentes- Comisión Nacional de Energía Atómica 
es mayor que en los anteriores. Para las concentraciones $x=35,40$ y $45 \%$ at. vemos un espectro complicado, que parece ser una distribución de campos hiperfinos. En el espectro de $\mathrm{Fe}_{35} \mathrm{Cu}_{65}$ todavía puede haber un doblete.

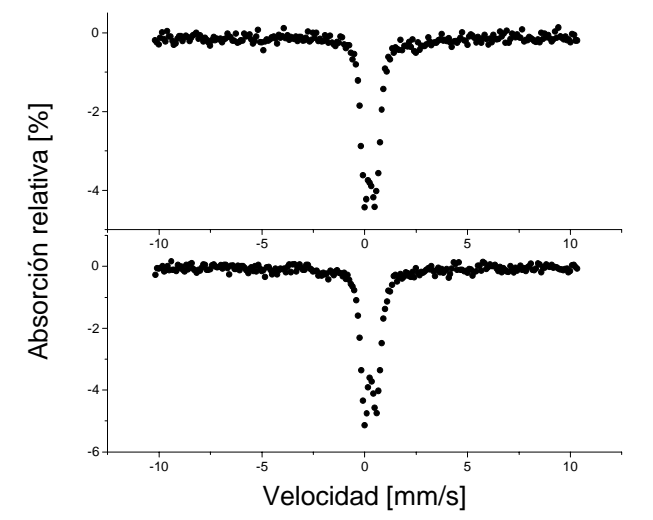

Fig. 16: Espectros Mössbauer de $\mathrm{Fe}_{10} \mathrm{Cu}_{90}$ (arriba), y de $\mathrm{Fe}_{14,1} \mathrm{Cu}_{85,9}$ (abajo); tomados
Las medidas Mössbauer tomadas a $77 \mathrm{~K}$ (fig. 16) en las muestras con $\mathrm{x}=10$ y $14,1 \%$ muestran que el doblete permanece casi sin alteraciones; a diferencia de [Ding 1995], en donde además del doblete también se identifica un sextete y un singlete correspondientes al $\alpha$-Fe y al $\gamma$ -

Fe, respectivamente. Como se vió en las medidas magnéticas, a esa temperatura y esas concentraciones el sistema se comporta paramagnéticamente, por lo que no es de extrañar que tengamos ese doblete.

Al medir esas mismas muestras a 4,2 $\mathrm{K}$ se puede ver un espectro complejo, proveniente de una distribución de campos hiperfinos (figura 17). Es posible distinguir en la medida de la muestra con $x=40 \%$ una contribución a altos campos proveniente de un óxido. El espectro de $\mathrm{Fe}_{40} \mathrm{Cu}_{60}$, que fue medido a $15 \mathrm{~K}$ muestra un

\begin{tabular}{|l|c|c|c|}
\hline Autor & $\mathrm{B}_{\mathrm{hf}}[\mathrm{T}]$ & $x$ & $\mathrm{~T}[\mathrm{~K}]$ \\
\hline Este trabajo & 20 & $\begin{array}{c}10, \\
14,140\end{array}$ & $\begin{array}{c}4,2 \\
15\end{array}$ \\
\hline [Ding 1995] & 20,3 & 25 & 77 \\
\hline [Tie 1995] & $\begin{array}{c}21 \\
24\end{array}$ & $\begin{array}{c}50 \\
60\end{array}$ & 300 \\
\hline [Jiang 1993] & 22 & 50 & 4,2 \\
\hline [Crespo 1993] & 22,3 & 51 & 300 \\
\hline [Macrí 1994] & 21,84 & 50 & 300 \\
\hline [Monteiro 1997] & 20 & 40 & 300 \\
\hline $\begin{array}{l}\text { [Sumiyama } \\
\text { 1983] }\end{array}$ & 22 & 30 & 4,2 \\
\hline
\end{tabular}
comportamiento similar a los dos mencionados. El campo hiperfino promedio es de alrededor de $20 \mathrm{~T}$. Este valor es comparable con los campos promedios reportados en diversos trabajos (ver Tabla 1). Larica et al reportan en 

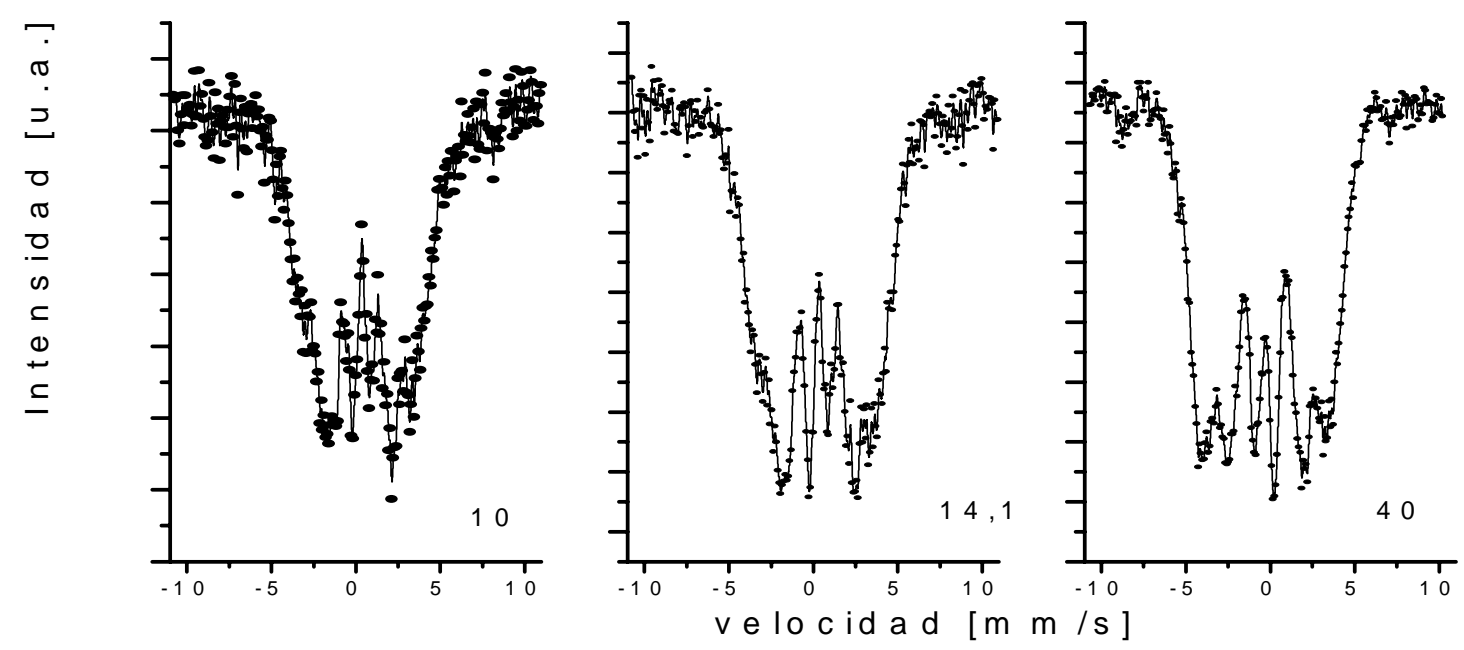

Fig. 17: Espectros Mössbauer tomados a muy bajas temperaturas. Los de $x=10$ y $14,1 \%$ fueron medidos a $4,2 \mathrm{~K}$ y el de $40 \%$ lo fue a $15 \mathrm{~K}$.

[Monteiro 1997] un campo promedio de 13,7 T, pero es visible en su figura 2 que una contribución importante está situada alrededor de 20 T. Este mismo campo ha sido reportado en muestras obtenidas por sputtering ([Sumiyama 1983]). Como vemos en la

Tabla 1, los campos reportados corresponden a muestras de muy distinta concentración, y medidos a diferentes temperaturas.

Las dos medidas a altas temperaturas tembién muestran un doblete ${ }^{34}$ (figura 14), con lo que vemos que los espectros complejos que presentaban a temperatura ambiente colapsan. En la figura 18 vemos el resultado de medir la absorción en función de la temperatura a una velocidad Döppler fija previamente seleccionada ${ }^{35}$. El comportamiento es decreciente con $\mathrm{T}$ hasta llegar a un valor constante. Es notable el cambio abrupto de comportamiento de estas curvas, que sugieren que en ese punto tenemos una temperatura crítica.

\footnotetext{
${ }^{34} \mathrm{La}$ medida de $\mathrm{Fe}_{45} \mathrm{Cu}_{55}$ fue realizada a una temperatura próxima a la de precipitación de otras componentes, con lo que los picos esbozados a velocidades altas pueden deberse al inicio de la precipitación de dichas fases (ver capítulo II).

${ }^{35}$ El procedimiento para seleccionar la velocidad fue el siguiente: Primero registramos en modo normal el espectro Mössbauer a temperatura ambiente y a una temperatura por encima de la $T_{c}$ predicha por la línea QuasiFM - PM del diagrama de fases magnéticas de §I.3.4.

Seleccionamos la velocidad en donde hubiera mayor absorción, lo que se correspondió con uno de los picos del doblete presente a altas temperaturas.
} 
En todos los espectros sería posible la presencia de un singlete correspondiente al $\gamma$-Fe, el cual sería levemente negativo. En todos los casos, si existe, este singlete está cubierto por la presencia de las otras componentes del espectro. Debido a las dificultades que presentan al ajuste los espectros de las muestras de concentración más alta, no ha sido posible dilucidar su presencia en el sistema. En todo caso ello indicaría que, si existiera, la fracción de Fe en $\gamma$-Fe sería muy pequeña. 
Para el análisis de estas medidas hemos utilizado un programa clásico de ajuste del espectro a lorentzianas (llamado ME) donde hay dobletes (o sea, los espectros de hasta $x=20 \%$ ). Para las muestras con $25 \%$ de Fe o más, el ajuste a los datos experimentales fue muy malo, aún infiriendo los sitios del hierro a partir de los resultados obtenidos en las muestras tratadas térmicamente ${ }^{36}$. En las muestras tratadas térmicamente el desorden es menor, y sitios que estaban tal vez esbozados en el espectro de las muestras "crudas" pudieron dar una señal más intensa debido a que crecieron en importancia relativa. Entonces, para las concentraciones de $x=25 \%$ atómico o más hemos calculado el corrimiento isomérico $\delta$ y el ensanchamiento magnético promedios, por medio de un programa que mide los momentos estadísticos

de los espectros Mössbauer. El programa toma en cuenta una región de referencia (los

${ }^{36} \operatorname{Ver} \S 11.3 .5$ 
canales de donde debe tomar el fondo), y la zona donde se encuentran los picos de absorción del espectro para calcular los momentos de diverso orden de la distribución. El momento de primer orden mide cuánto se aparta del canal de velocidad cero el centro de masa del espectro de absorción en conjunto, dando así una medida del corrimiento isomérico del sistema como un todo (es decir, correspondería a un promedio ponderado del corrimiento isomérico de cada subespectro). El momento de segundo orden mide cuán expandido está el espectro, tomando como origen el corrimiento isomérico promedio calculado. De esta manera, en ausencia de un modelo apropiado para el ajuste, disponemos de un parámetro que permite cuantificar la expansión espectral a velocidades mayores. El momento de segundo orden $\mathrm{m} 2$ reflejará así la aparición y

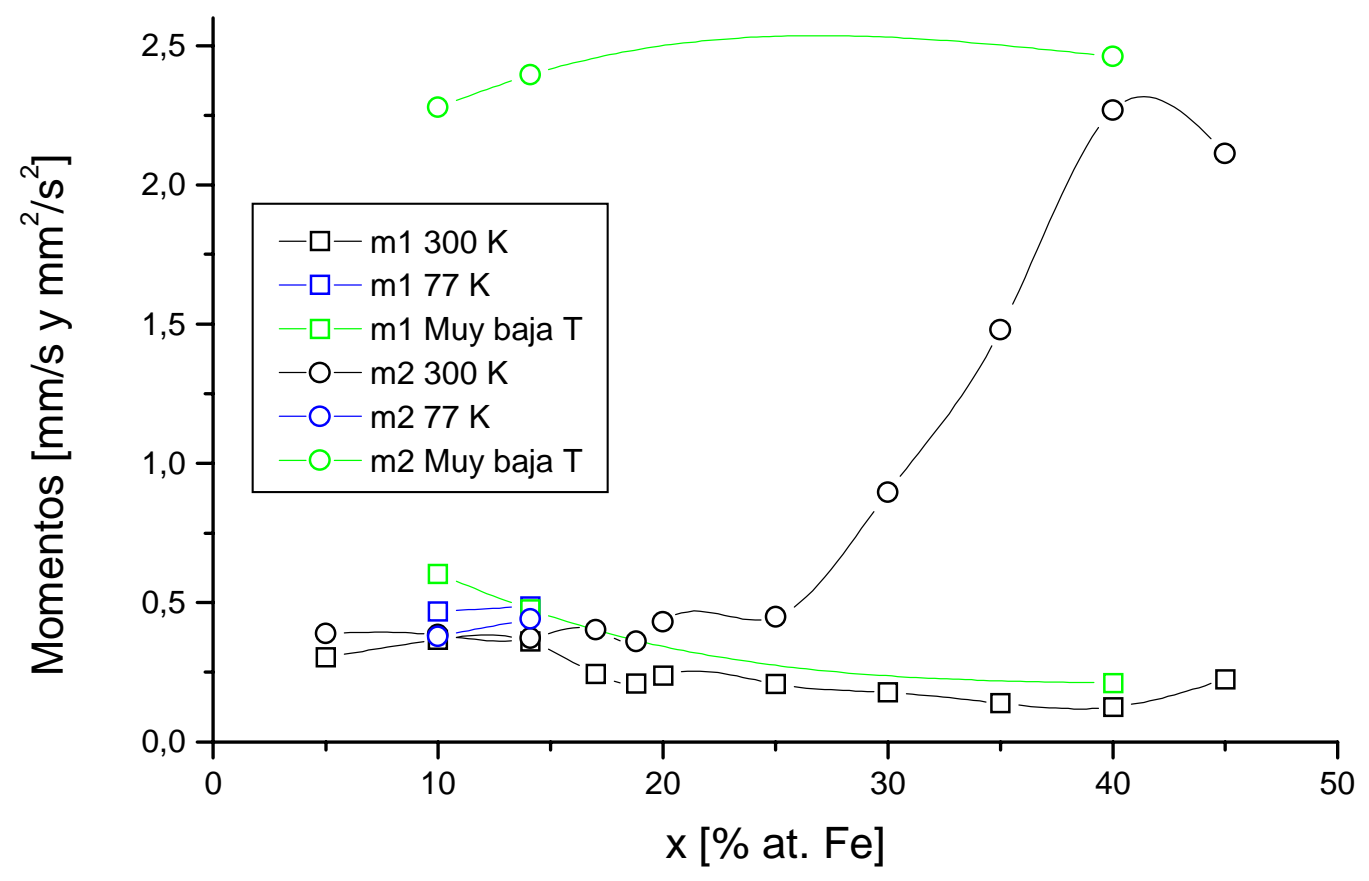

Fig. 19: Medida del corrimiento isomérico promedio $\mathrm{m} 1 \mathrm{a}$ temperatura ambiente (cuadrados negros), y a bajas temperaturas (cuadrados azules y verdes). Los círculos representan el nromedio del camno maonético hinerfinn $\mathrm{m}$ ) a temneratura amhiente (neornc) y a haias desarrollo de componentes magnéticas. Los resultados se presentan en la figura 19. 
El corrimiento isomérico m1 muestra un comportamiento decreciente con la concentración. Alcanza un valor máximo de $0,29 \mathrm{~mm} / \mathrm{s}$ para la muestra con $x=14,1 \%$. Luego $\delta$ disminuye. En general, los valores obtenidos son altos en comparación con los reportados en [Chien 1986]. Agregamos en la figura los valores obtenidos del ajuste en las muestras medidas a alta temperatura.

Hemos analizado el comportamiento de $\delta$ con la concentración. Con este fin consideramos que el corrimiento isomérico tiene una parte local $\left(\delta_{1}\right)$, debida al entorno químico inmediato al átomo; y otra no local $\left(\delta_{\mathrm{nl}}\right)$, debida a la influencia del resto de los átomos:

$$
\delta(c)=\delta_{l}(c)+\delta_{n l}(c)
$$

Ambas partes son función de la fracción atómica $c$. La parte local debe reflejar la configuración de vecinos hierro y cobre. Consideramos entonces que esta parte es una función decreciente de la concentración, con la siguiente forma:

$$
\delta_{l}(c)=\left[12-n_{F e}(c)\right] \cdot 0,016,
$$

con $n_{F e}$ el número medio de vecinos

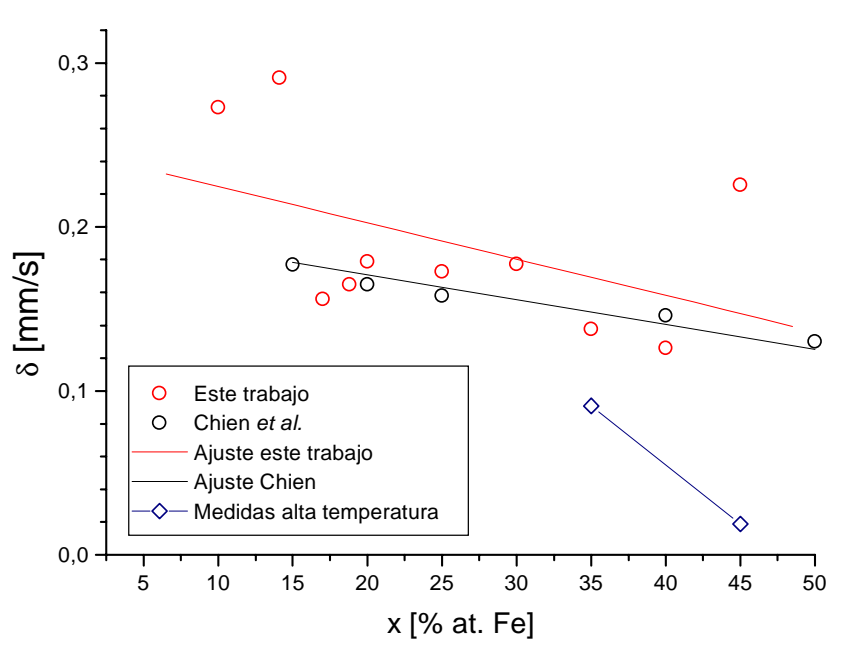

Fig. 20: Corrimientos isoméricos de nuestras muestras (círculos rojos) y las de Chien (círculos negros), con los respectivos ajustes (líneas). El acuerdo entre ambas es hıeno Fn azıl incluímos loc corrimientos isomérienc de

hierro. El valor de $0,016 \mathrm{~mm} / \mathrm{s}$ corresponde al $\delta$ para un átomo de $\mathrm{Fe}$ con un único vecino $\mathrm{Cu}$ en la primera esfera de coordinación, medido respecto al $\alpha$-Fe [Shenoy 1978]. Cada átomo de hierro agregado disminuirá el corrimiento isomérico hasta hacerlo aproximadamente nulo cuando todos los átomos vecinos sean hierros. 
Suponiendo una distribución binomial (solución sólida aleatoria) el número medio $n_{F e}(c)$ es simplemente la concentración relativa multiplicada por 12. Así, la parte local será:

$$
\delta_{l}=0,192(1-c)
$$

La parte no local es una función creciente de la concentración relativa, con las condiciones:

$$
\begin{aligned}
& \delta_{n l}(0)=0 \\
& \delta_{n l}(1)=0,063
\end{aligned}
$$

donde el último valor es el corrimiento isomérico que debiera tener una muestra cuando la muestra es completamente hierro fcc $^{37}$, asumiendo una variación lineal. Así, la parte no local del corrimiento isomérico será:

$$
\delta_{n l}=0,063 \cdot c
$$

Combinando ambas llegamos a que

$$
\delta(c)=0,192-0,13 \cdot c
$$

Ajustando los corrimientos isoméricos de nuestras muestras a una recta, obtenemos

$$
\delta(c)=0,247-0,22 \cdot c,
$$

en razonable acuerdo con las medidas de Chien (ver fig. 19). Debemos destacar que a las concentraciones donde el efecto magnetoresistivo es mayor $(17 \leq x \leq 40)$, nuestros corrimientos isoméricos siguen muy

${ }^{37}$ Para calcular este valor ajustamos los corrimientos obtenidos por Chien en la zonta ${ }^{\left[K_{k}\right.} \mathrm{cc}$ con una recta y extrapolamos a $100 \%$ atómico. El ajuste nos dio $\delta=0,193-0,13 x$. Estamos

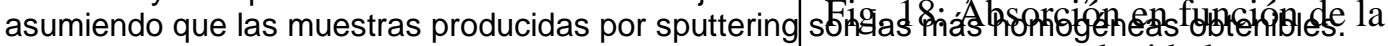
temperatura, a velocidad constante. Arriba: Fan.ru.. Ahoin. Fo..Cun Con 1o flanho 
bien a la recta ajustada con los valores de Chien et al. Podemos afirmar entonces, dado que el corrimiento isomérico es una medida del entorno químico, que nuestras muestras son ligeramente más inhomogéneas que las obtenidas por sputtering.

Al medir a bajas temperaturas $\langle\delta>$ alcanza valores más elevados. El corrimiento isomérico para $\mathrm{Fe}_{10} \mathrm{Cu}_{90}$ es de $0,57 \mathrm{~mm} / \mathrm{s}$, y decrece con la concentración. Los valores más altos pueden deberse exclusivamente al corrimiento Döppler de segundo orden.

El momento de segundo orden $\mathrm{m} 2$ sugiere que el campo magnético hiperfino promedio es aproximadamente nulo hasta $x=25 \%$, a partir de donde crece en forma aproximadamente lineal (fig. 19). A muy bajas temperaturas $B_{h f}$ se mantiene aproximadamente constante con la concentración.

Podemos explicar el comportamiento del campo magnético hiperfino con la concentración y la temperatura pensando que, si se trata de pequeñas partículas con un cierto momento magnético (producido esencialmente por el hierro), en la medida en que se encuentran próximas unas con otras se acoplan magnéticamente (vía la interacción de

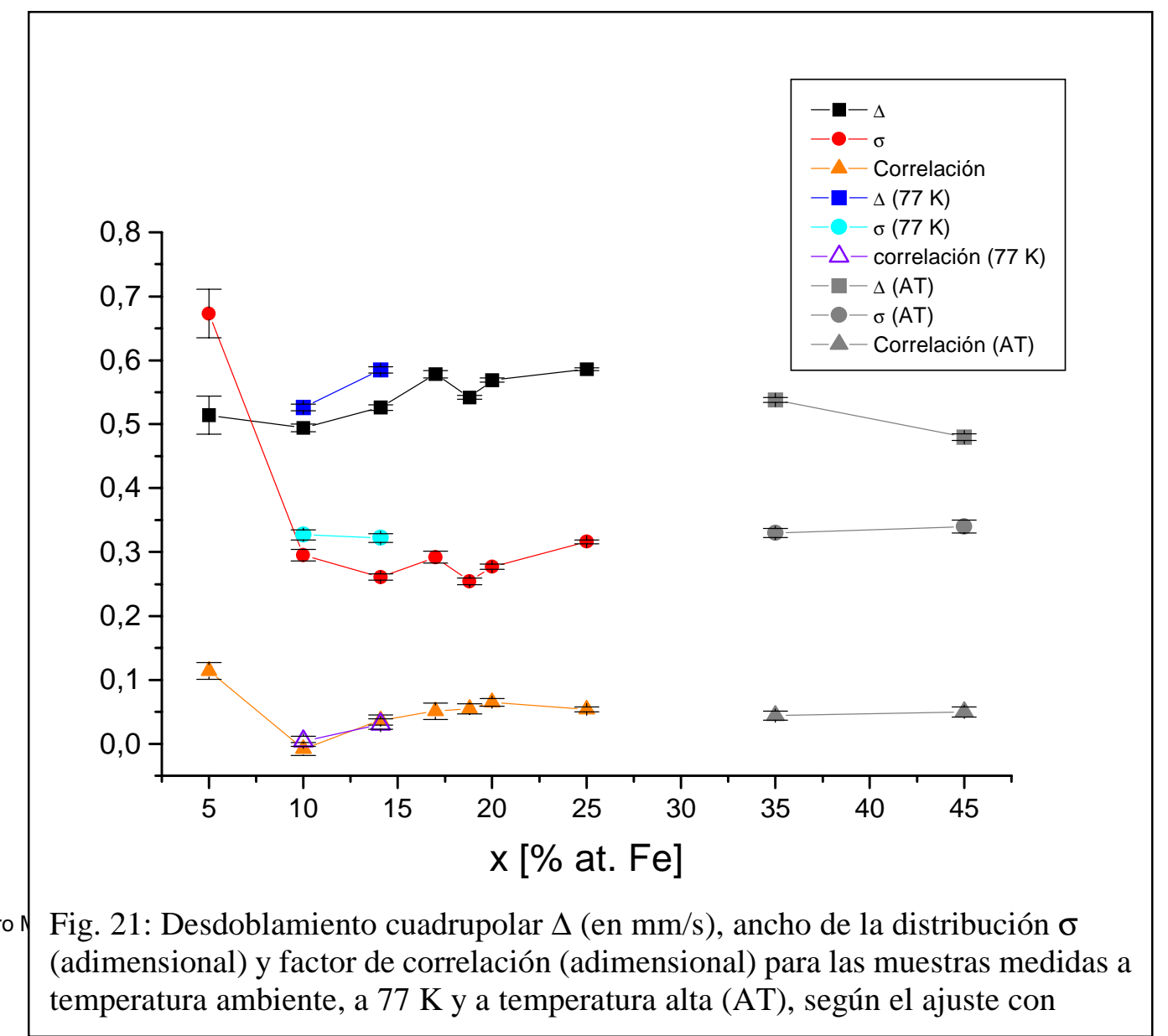


intercambio o la dipolar). Esto impide la rotación de sus momentos, que es causada por la agitación térmica. Para cada temperatura este acoplamiento ocurre para una cierta concentración, ya que a menores $x$ las partículas no se encuentran suficientemente cerca para acoplarse, por lo que les es más fácil relajar superparamagnéticamente ${ }^{38}$. Según las medidas a temperatura ambiente, este acoplamiento ocurre a partir de $x \approx 25 \%$. Para ser más precisos, a temperatura ambiente esas partículas (que pueden ser los clusters de los que hemos hablado antes, o bien clusters en contacto) son suficientemente grandes como para que dejen de ser "paramagnéticas". Los clusters en las muestras $\mathrm{Fe}_{10} \mathrm{Cu}_{90}$ y $\mathrm{Fe}_{14,1} \mathrm{Cu}_{85,9}$ son lo suficientemente pequeños o están tan alejados entre sí como para que el espectro Mössbauer continúe colapsado a $77 \mathrm{~K}$. A muy bajas temperaturas vemos que las magnitudes de $\mathrm{B}_{\mathrm{hf}}$ de los tres espectros medidos son prácticamente los mismos: podemos pensar que no hay un cambio sustancial en la composición y/o morfología de los clusters. Y que efectivamente el aumento del campo con la concentración se debe al acoplamiento entre clusters, ya que la tendencia es a alcanzar el valor de muy bajas temperaturas, que es el del cluster "congelado" magnéticamente. Esta interpretación es reforzada por las medidas obtenidas a altas temperaturas de $\mathrm{Fe}_{35} \mathrm{Cu}_{65}$ y de $\mathrm{Fe}_{45} \mathrm{Cu}_{55}$. El ajuste de estos dobletes da como resultado parámetros hiperfinos consistentes con los de los dobletes de concentraciones más bajas medidos a temperatura ambiente. Los corrimientos isoméricos de los primeros quedan por debajo de la tendencia de los obtenidos en los segundos (ver figura 20), debido esencialmente al corrimiento Döppler térmico de las líneas.

El desdoblamiento cuadrupolar $\Delta$ de los dobletes crece ligeramente con la concentración, como podemos ver en la figura 21. Su valor está en un rango que va de 0,514 a $0,586 \mathrm{~mm} / \mathrm{s}$, con un valor medio de $0,544 \pm 0,035 \mathrm{~mm} / \mathrm{s}$. Este valor es

\footnotetext{
${ }^{38}$ Ver parágrafo $§ 4$
} 
comparable con el reportado en [Ding 1995] $(\Delta=0,49 \mathrm{~mm} / \mathrm{s})$ y en [Monteiro 1997] $(\Delta=$ $0,51 \mathrm{~mm} / \mathrm{s}$ ), mayor al obtenido por Elkakouly et al ([Elkakouly 1995], $\Delta=0,36 \mathrm{~mm} / \mathrm{s}$ ) y menor al mostrado en [Macrí 1994], $(\Delta=0,95 \mathrm{~mm} / \mathrm{s})$. Estos valores corresponden a muestras de diferentes concentraciones. El aumento de $\Delta$ con la concentración ocurre a pesar del aumento del parámetro de red, y puede ser debido a un ligero enriquecimiento en Fe de los entornos de la sonda. Como $\Delta$ es una medida de la distorsión del entorno de primeros vecinos respecto a uno cúbico ${ }^{39}$, este aumento indicaría que los primeros vecinos hierro son un poco menores o mayores que cuatro (o seis). Del ajuste de los dobletes con una distribución voigtiana (una distribución gaussiana de líneas lorentzianas ${ }^{40}$ ) también obtuvimos el ancho de la distribución $\sigma$ y el factor de correlación entre $\Delta$ y $\delta$. El ancho de la distribución se mantiene dentro de un valor $\sigma=$ $0,34 \pm 0,15$, lo que indica que no hay una gran dispersión en los sitios, en otras palabras, que los entornos son bastante parecidos para cualquiera de los átomos de hierro. El factor de correlación se mantiene positivo, lo que está diciendo que $\Delta$ cambia en el mismo sentido que $\delta$. Las dos medidas a $77 \mathrm{~K}$ muestran el mismo comportamiento que las medidas a temperatura ambiente. El desdoblamiento cuadrupolar es levemente más grande, como se espera para una medida a menor temperatura. Las medidas a altas temperaturas de $\mathrm{Fe}_{35} \mathrm{Cu}_{65}$ y de $\mathrm{Fe}_{45} \mathrm{Cu}_{55}$ tienen parámetros $\sigma$ y de correlación similares a los dobletes de menor concentración, como vemos en la figura 21. Esta es otra indicación de que las muestras de mayores concentraciones pueden tener clusters de tamaño similar a las de bajas concentraciones.

\footnotetext{
${ }^{39} \Delta$, que es proporcional al gradiente del campo eléctrico, tiene que anularse tanto para cero $u$ ocho (doce) vecinos hierro en una red bcc (fcc). Por tanto, $\Delta$ debe tener un comportamiento parabólico, con su máximo para cuatro - cinco (seis - siete) vecinos hierro.

${ }^{40}$ Las líneas lorentzians corresponden al proceso de absorción resonante nuclear, que es la base del efecto Mössbauer. Al usar una distribución voigtiana estamos asumiendo que los sitios de la sonda Mössbauer (el ${ }^{57} \mathrm{Fe}$ ) se encuentran distribuidos gaussianamente
} 
Podemos resumir en que lo visto por espectroscopía Mössbauer nos habla de un sistema de clusters ricos en hierro de concentración aproximadamente constante, los cuales a bajas temperaturas producen espectros magnéticamente desdoblados con un campo promedio de $20 \mathrm{~T}$. A concentraciones menores al $25 \%$ estos clusters son superparamagnéticos a temperatura ambiente. A concentraciones mayores, o bien tenemos "grumos" de clusters de tamaño fijo, que están acoplados directamente (con interacción de intercambio y/o dipolar), o bien clusters de tamaño creciente con la concentración. En cualquiera de los dos casos el efecto es el de "ralentizar" el salto de su espín entre sus ejes fáciles. Un incremento en la temperatura vuelve a desacoplarlos.

Esta interpretación explica también por qué los valores del campo hiperfino medidos por otros autores son bastante similares entre sí, a pesar de las diferencias en la concentración de hierro global. 


\subsection{Dispersión de rayos $\mathrm{X}$ a bajos ángulos (SAXS)}

En esta sección daremos cuenta de los resultados obtenidos por medio de la dispersión de rayos $\mathrm{X}$ a bajos ángulos (SAXS). Los principios de la misma serán desarrollados en el Apéndice B. Esta técnica nos permitió determinar la microestructura de las aleaciones. Realizamos las medidas en el Laboratorio Nacional de Luz Síncrotron (LNLS - Campinas), en la estación SAXS. Colocamos las muestras en un portamuestras (10 $\mathrm{mm}$ por $3 \mathrm{~mm}$, con un espesor de $0,4 \mathrm{~mm}$ ) y las envolvimos en cinta plástica. Tomamos las medidas en geometría de transmisión con un detector sensible a la posición vertical. Efectuamos los registros con dos longitudes de cámara: larga (1,5 m) y corta $(0,6 \mathrm{~m})$, esta última con un cortador de haz ancho. Esto nos permitió registrar las regiones $0,0052<k<0,2$ y $0,2<k<0,45 \AA^{-1}$. Los espectros corregidos registrados con la cámara de 0,6 m presentan una fuerte dispersión alrededor de $k \approx 0,4 \AA^{-1}$ debida al Kapton, con lo que no fue posible efectuar una buena sustracción de la dispersión parásita. Pero sí indican que la ley de Porod continúa en este rango de $k$ sin ninguna otra característica adicional.

Los espectros (fig. 22) muestran un decrecimiento suave con $k$ en todo el rango medido, como así también un decrecimiento de la intensidad con la concentración de Fe. Se ve que a valores de $k$ grandes (región de Porod) los espectros siguen una ley $k^{-4}$, tanto en los tomados con cámara larga como corta (ver fig. 23). Esto indica que las interfaces de las inhomogeneidades son marcadas y que el sistema puede ser considerado como particulado y tridimensional.

Los gráficos de Guinier $\left(\ln (I)\right.$ vs. $\left.k^{2}\right)$ son cóncavos para todas las medidas (ver fig. 24) indicando polidispersidad, es decir, que no existe una longitud característica única para las inhomogeneidades composicionales, excepto para $k$ pequeños, donde vemos que la pendiente es acotada. Esta recta nos permite estimar un 


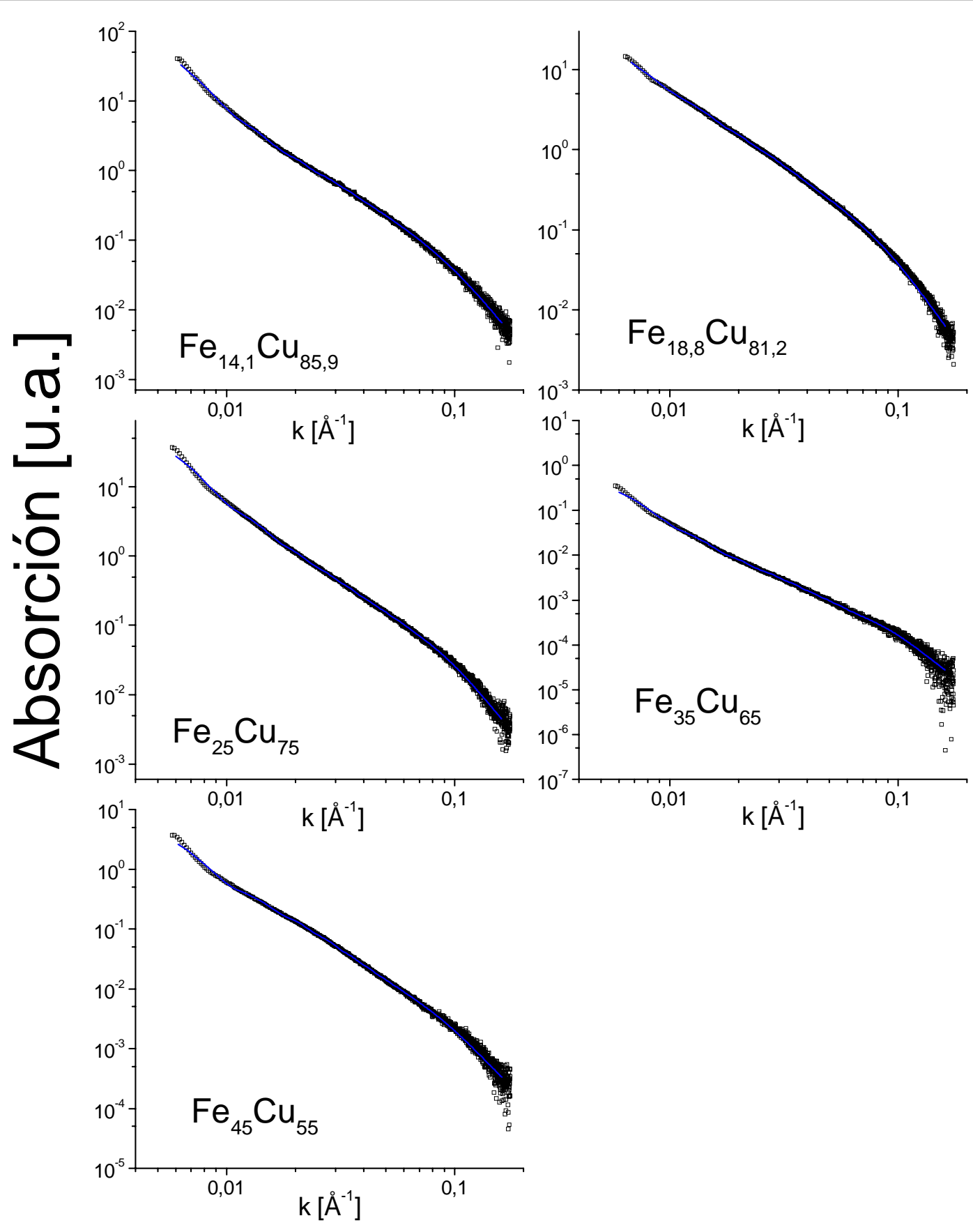

Fig. 22: Gráficos log-log de la intensidad vs.el vector de onda $k$. Las ordenadas están en unidades arbitrarias. El ajuste correspondiente está incluido como una línea azul. I ns nuntos exnerimentales están marcados como cı ıdrados abiertos

valor del radio de giro $R_{g}$, que asumiendo inhomogeneidades esféricas está relacionada

con el radio $R$ a través de $R=\sqrt{5 / 3} R_{g}$. Este radio oscila entre 33,7 y 45,0 nm y asumimos que está relacionado con dispersiones no deseadas originadas en las 
imperfecciones de la superficie macroscópica de la muestra [Roth 1977, Liu 1978].

Descartamos en nuestros análisis una contribución de los poros de los granos de polvo resultantes ${ }^{41}$. Una estimación grosera del tamaño de los poros conduce a una longitud de alrededor de $360 \mathrm{~nm}$, si consideramos que los granos macroscópicos tienen un tamaño característico de $1 \mu \mathrm{m}$. Estas longitudes están muy por fuera de nuestro rango de detección.

De los comportamientos en las zonas

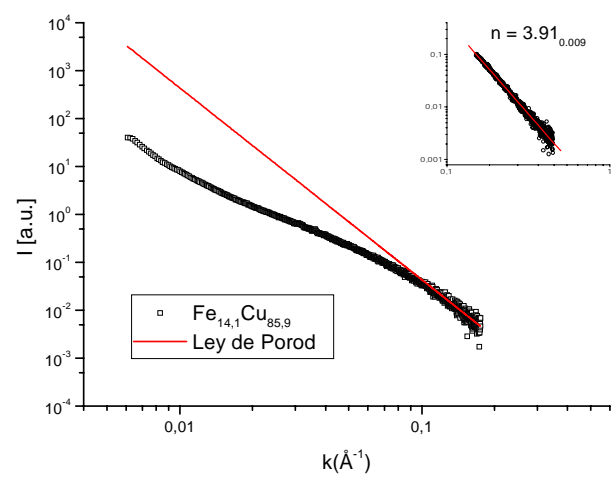

Fig. 23: El espectro, en la región de grandes $\mathrm{k}$, ajusta a una ley de

de Porod y Guinier inferimos que la aleación está constituida por una colección de objetos que dispersan distribuidos en una matriz. Podemos relacionar la dispersión, en el

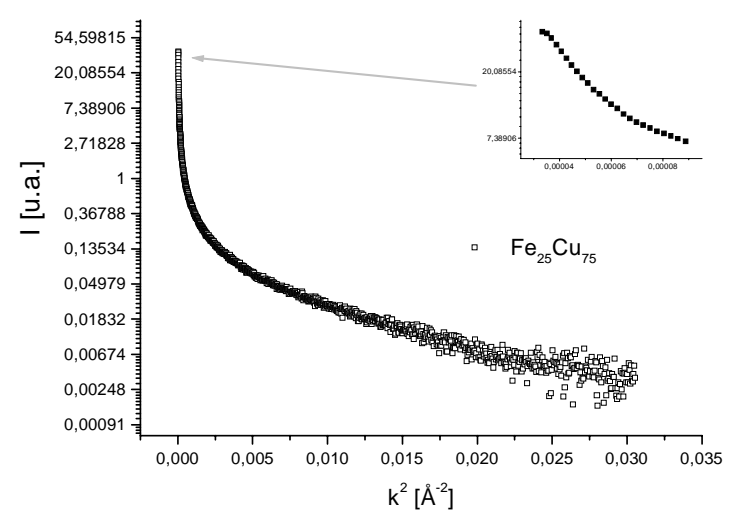

Fig. 24: Gráfico de Guinier. Las imperfecciones macroscópicas dan lugar a una pendiente acotada a valores pequeños de $\mathrm{k}$ (inserto). espacio recíproco, con una distribución volumétrica de partículas a través de:

$$
I(k)=\int_{0}^{\infty}\left\langle F^{2}(k R)\right\rangle D_{v}(R) d R
$$

donde $F(k R)$ es el factor de forma de las inhomogeneidades y $D_{V}(R)$ es la distribución volumétrica. Asumimos conocido el factor $F$ para determinar $D_{V}$. Utilizamos un

\footnotetext{
${ }^{41}$ No confundir con los granos de los que hablamos en §I.3.3, que son los cristales o cristalitas. En este caso estamos hablando de los granos macroscópicos.
} 
programa de ajuste numérico, el GNOM ([Svergun 1992]), con el cual ajustamos una distribución volumétrica de partículas esféricas de radio $\mathrm{R}$ a los datos experimentales. La distribución volumétrica se define como:

$$
D_{V}(R)=4 \pi R^{3} N(R) / 3
$$


donde $N(R)$ es el número relativo de dichas esferas. Los ajustes obtenidos se presentan en la figura 25. Como vemos, la mayor población está situada a radios pequeños. Hay una contribución a $R=45 \mathrm{~nm}$ que corresponde a la zona de $k$ más pequeños, la cual es ficticia, ya que correspondería a la

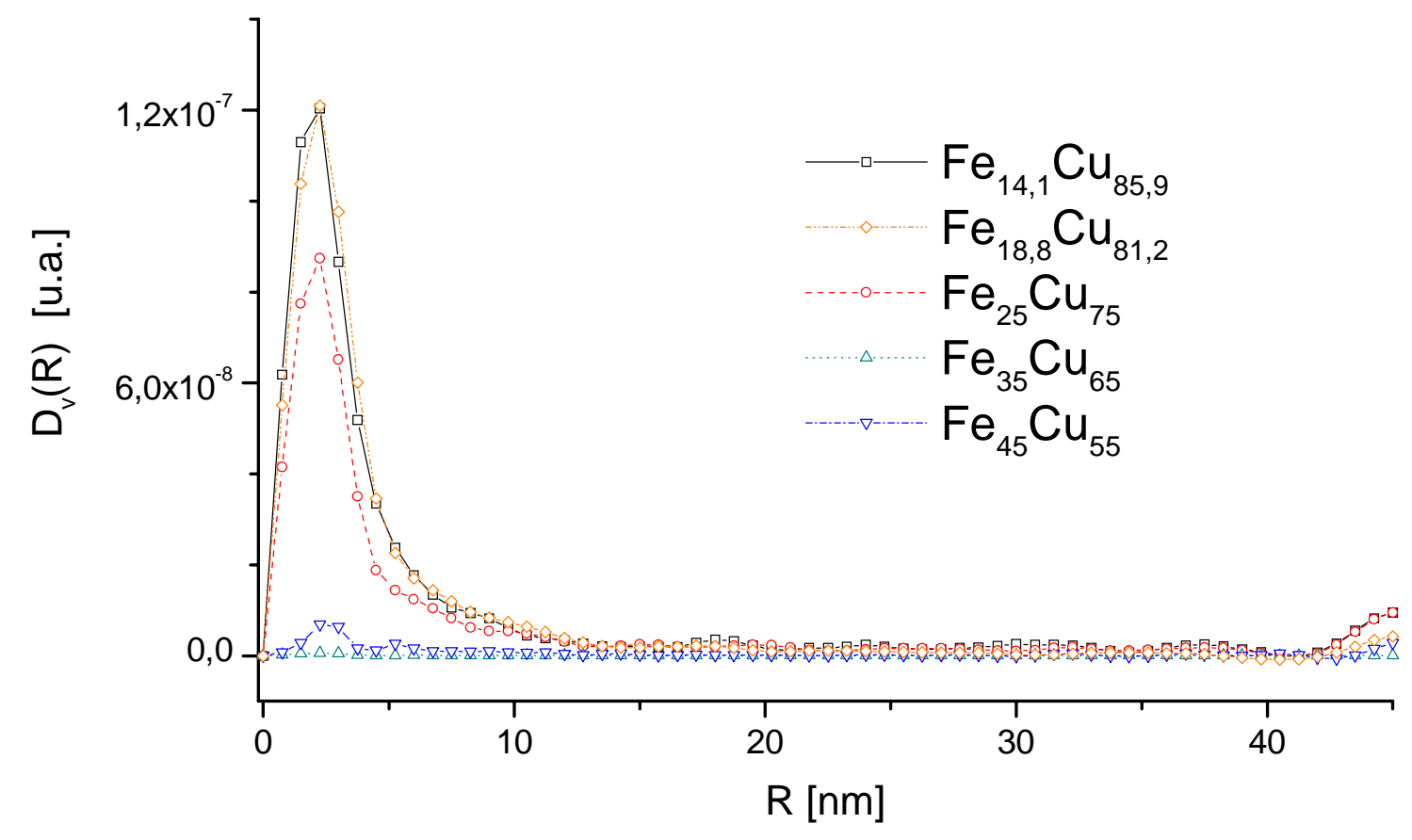

Fig. 25: Distribuciones volumétricas de esferas, según el ajuste con GNOM.

dispersión en las superficies, como mencionamos en el análisis de los gráficos de

Guinier. La parte de la distribución que refleja auténticamente a las inhomogeneidades es la de radios de hasta $15 \mathrm{~nm}$. Deconvolucionamos a estas distribuciones en componentes mediante el ajuste de curvas gaussianas ${ }^{42}$. Los centroides de estas gaussianas se muestran en la figura 26 en función de la concentración. Podemos identificar tres tamaños característicos, de 2, 6 y $10 \mathrm{~nm}$. El primero de ellos es el que da

\footnotetext{
${ }^{42}$ Intentamos un ajuste con funciones lognormales, que son las normalmente usadas para pequeñas partículas, pero esto no fue posible sin utilizar menos de seis de estas funciones, es decir el doble de la cantidad utilizada de gaussianas. Por otro lado, estamos interesados en contar la cantidad de partículas que hay alrededor de un cierto tamaño.
} 
cuenta de la mayor cantidad de partículas, como se ve en la figura 27. Si calculamos el radio medio de las partículas, pesado con su distribución volumétrica, vemos que se mantiene dentro de un valor de $3,77 \pm 0,31 \mathrm{~nm}$, en buen acuerdo con los tamaños de

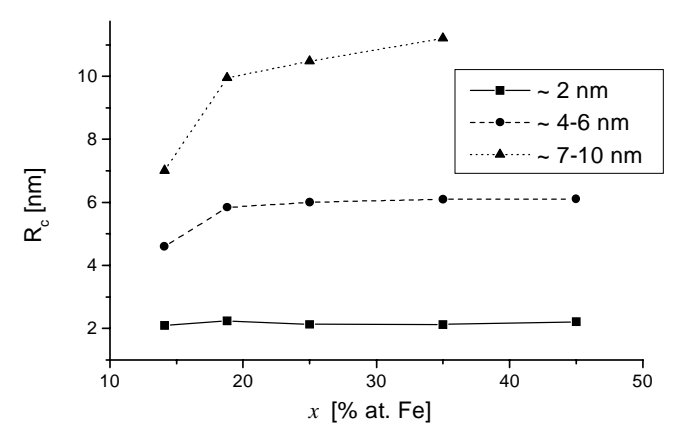

Fig. 26: Posición de los centroides de las gaussianas entre 3 y $40 \mathrm{~nm}$ estimados en diversos sistemas magnetoresistivo gigantes ${ }^{43}$.

Una parte de la dispersión puede deberse a defectos y/o la gran densidad de bordes de grano siempre presentes en los materiales nanocristalinos. No

podemos separar esta dispersión de la debida a fluctuaciones en la composición, pero

como obtodas las muestras estudiadas habían sido sometidas al mismo tiempo de tratamiento mecánico, asumimos que las diferencias entre patrones se deben sólo a las diferencias composicionales.

Analizamos la naturaleza de las inhomogeneidades observadas con el modelo

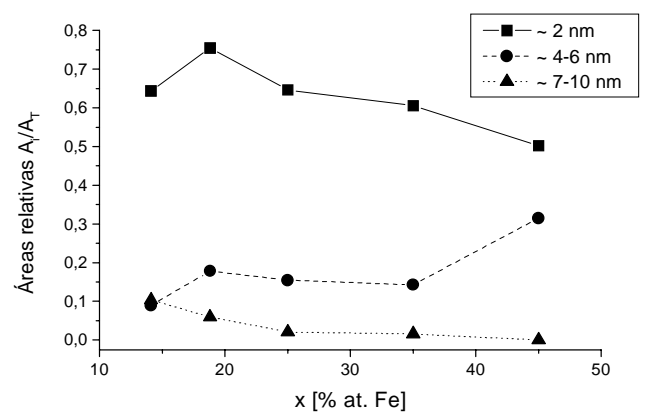

Fig. 27: Fracciones relativas de cada tamaño característico general de dos fases. La intensidad integrada o invariante $Q$ en este modelo está relacionada con las densidades electrónicas $\rho_{l}$ y $\rho_{2}$ mediante:

$$
Q=\int_{0}^{\infty} I(k) k^{2} d k=2 \pi^{2}\langle\Delta \rho\rangle^{2} V
$$

donde $\langle\Delta \rho\rangle^{2}=\left(\rho_{1}-\rho_{2}\right)^{2} c(1-c)$,

\footnotetext{
${ }^{43}$ Dependiendo del sistema, del método de preparación y del posterior tratamiento térmico. Consultar [Wang 1994], [Dupuis 1997] o [Yasuna A].
} 
con $c$ y $(1-c)$ las fracciones volumétricas de cada fase.

El invariante $Q$ se obtiene de los datos ([Raap 1995]) a través de:

$$
Q=\frac{\left(k_{i}\right)^{3} I\left(k_{i}\right)}{2}+\operatorname{sum} \Delta k+\frac{A}{k_{f}},
$$

donde $k_{i}$ es el valor de $k$ más pequeño, $I\left(k_{i}\right)$ la intensidad para ese valor, sum es la suma de Riemann efectuada sobre la curva en el gráfico de Guinier, $\Delta k$ es el paso de la medición, $A$ es el valor obtenido en la fórmula (1) del Apéndice $\mathrm{B}, \mathrm{y} k_{f}$ el valor de $k$ medido más grande. Estos tres términos representan el intervalo $\left[0, k_{\mathrm{i}}\right]$, la parte central del espectro, y el intervalo $\left[k_{\mathrm{f}}, \infty\right)$ para el cálculo de la integral (2). Asumimos que la intensidad correspondiente a los valores más pequeños de $k\left(\left[0, k_{\mathrm{i}}\right]\right)$ tenía una forma triángular.

Vemos los resultados de este cálculo en la figura 28. Se puede obtener el volumen fraccional de los entes dispersantes a partir del valor de $Q$, provisto que tengamos un solo contraste. A pesar de que no hay indicación de un contraste único, el invariante $Q$ decrece con la concentración, lo que estaría indicando que el grado de inhomogeneidad decrece cuando $x$ crece hacia $50 \%$. En 35 y $45 \%$ at. $Q$ es casi cero, como vemos en la figura mencionada. Discutiremos el comportamiento de $Q$ con la concentración de Fe con un modelo general de dos fases, en el cual la fase rica en Fe en la matriz del $\mathrm{Cu}$ tiende a desaparecer para $x \rightarrow 50 \%$, debido a la creciente homogeneidad de la aleación.

En los estudios Mössbauer y de DRX no se detectó $\alpha$ - o $\gamma$-Fe. Los patrones XRD solamente muestran las líneas de difracción del Cu ligeramente corridas hacia menores ángulos; lo que significa un incremento en el parámetro de red; y el ensanchamiento de las líneas de reflexión, debido a tensiones y la disminución en el tamaño de grano. Esto indicaría una solución sólida homogénea. Por otra parte, el efecto 
MRG fue detectado en ese rango de composiciones, con un máximo en $x=20 \%$, lo que sólo podemos explicar suponiendo la existencia de partículas magnéticas inmersas en una matriz no magnética, o sea que esos clusters deben ser diferentes en composición a su entorno, con lo cual es esperable un contraste con la matriz. En acuerdo con esta observación, nuestros resultados SAXS indican que las aleaciones son inhomogéneas, con una distribución de tamaños de hasta $15 \mathrm{~nm}$. Nuestros datos SAXS están de acuerdo con una estructrura particulada y no con una láminar, ya que en este caso se esperaría una ley de potencias $k^{-2}$ previa a la región de Porod ([Porod 1982]). Tomando en cuenta las ecuaciones 2 y 3 , el invariante medido sólo puede ser explicado en un modelo donde $\left(\rho_{1}-\rho_{2}\right)^{2}$ decrece monótonamente en el rango $0 \leq x \leq 50 \%$. Intentamos obtener una descripción cualitativa de cómo la composición de la matriz y las inclusiones deben variar para dar cuenta de los valores de $Q$ medidos. Con este fin hemos asumido en el rango $0 \leq x \leq x_{0}$ composiciones medias $\mathrm{Cu}_{100-\mathrm{q}} \mathrm{Fe}_{\mathrm{q}}$ y $\mathrm{Cu}_{\mathrm{p}} \mathrm{Fe}_{100-\mathrm{p}}$ para la matriz y las inclusiones, respectivamente. Para el rango $x_{0} \leq x \leq 50 \%$ se eligió $\mathrm{Cu}_{100-\mathrm{x}} \mathrm{Fe}_{x}$ para ambas, matriz e inclusiones. Luego evaluamos

$\langle\Delta \rho\rangle^{2}=\left(\rho_{1}-\rho_{2}\right)^{2} x(100-x)$ como $D(x-q)(100-p-q)$, donde $D$ es una constante a ser determinada. Para poder comparar los valores de $Q$ calculados y medidos propusimos dependencias específicas de $q$ y 100-p con $x$ :

$$
\begin{aligned}
& q=C x^{n} \\
& 100-p=A+B x^{m}
\end{aligned}
$$

con la condición de borde

$$
q\left(x_{0}\right)=100-p\left(x_{0}\right)=x_{0}
$$


Estas condiciones más los cinco puntos experimentales son suficientes para determinar los siete valores desconocidos $A, B, C, D, n, m$ e $y_{0}$. La figura 28 muestra el mejor ajuste obtenido. En el inserto de esa figura mostramos la dependencia con $x$ de la composición de la matriz y las inclusiones. Los resultados indican inhomogeneidades con concentración de $\mathrm{Fe}$ igual a $35 \%$ at. con contraste constante entre matriz e inhomogeneidad. Existen en la literatura pocos reportes acerca de la composición de los clusters. Por ejemplo, las observaciones a las medidas Mössbauer, que adjudican a que los átomos de $\mathrm{Fe}$ tienen algunos vecinos cobre el hecho de que el campo magnético hiperfino es bien diferente al del $\alpha$-Fe. En la serie de trabajos de Huang et al. ([Huang 1996, 1998]) se hacen

observaciones a la composición de los granos producidos por el MM.

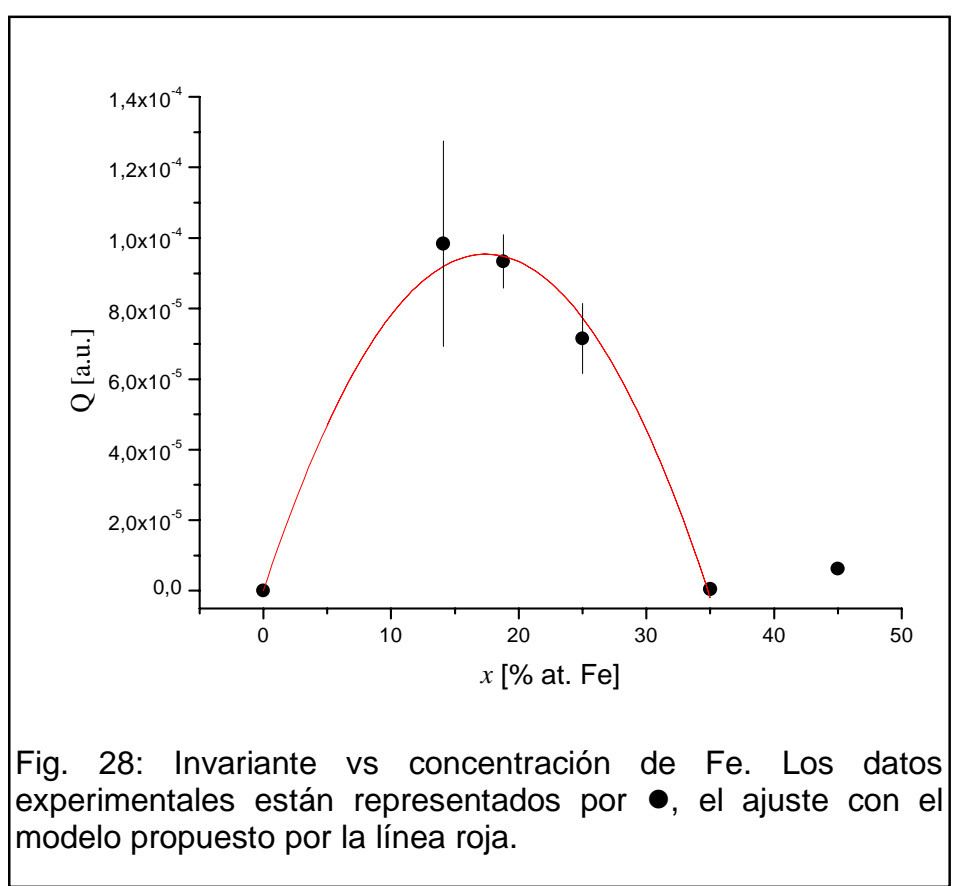




\section{Discusión y conclusiones}

Según se entiende actualmente al molido mecánico, el proceso continuo de fragmentación, soldadura y deformación de las partículas de polvo de $\mathrm{Fe}$ y $\mathrm{Cu}$ da lugar al mezclado a nivel atómico. Los trabajos de Huang et al. ${ }^{44}$ han mostrado que es posible una explicación (compatible con lo propuesto por Schwarz en [Schwarz 1998]) donde la incorporación de átomos de cobre a los granos de hierro va haciendo que, en ellos, la fase bcc $(\alpha-\mathrm{Fe})$ se vuelva inestable. Para una concentración del $20 \%$ atómico de cobre el grano hace una transformación martensítica y se transforma en fcc. El trabajo mecánico también produce una reducción en el tamaño de grano. Conforme transcurre el tiempo de molido, los granos de cobre y hierro alcanzan un tamaño similar $(20 \mathrm{~nm}$, aproximadamente ${ }^{1}$ ). Ambos tienen ahora la misma estructura y prácticamente el mismo parámetro de red. Entonces el trabajo mecánico colabora en su interdifusión. De esta manera se alcanza una mezcla fccFe-Cu relativamente homogénea. Esta homogeneidad es limitada, debido a que la entalpía de formación de la mezcla es positiva. Esto determina que la aleación sea metaestable, por lo que un ulterior aporte de energía en forma de calor separará casi en su totalidad a los elementos en sus formas originales. Nuestros difractogramas confirman que, hasta lo detectable por la técnica de DRX, las aleaciones resultantes son fcc en todo el rango de concentraciones estudiado $(1 \leq x \leq 45$ \%). Por espectroscopía Mössbauer tampoco hemos visto $\alpha$-Fe, ni siquiera en estado SPM, así como tampoco carburos. El parámetro de red aumenta con la concentración, y esto es atribuido a partir del trabajo de Yavari $^{45}$ a efectos de magnetovolumen. Nuestras muestras tienen un parámetro de red ligeramente más alto que lo anteriormente reportado, debido tal vez al uso de otra técnica de cálculo, o bien a la presencia de

44 [Huang 1994, 1996, 1998], ver §l.1. Trabajos previos.
${ }^{45}$ [Yavari 1992] 
átomos de carbono producto de la descomposición del agente antiadherente. Los átomos de carbono estarían como intersticiales, dilatando ligeramente la red fcc. En un molino de más alta energía que el usado para preparar el conjunto original de muestras, se forman carburos luego de procesamientos prolongados ${ }^{46}$. Entendemos entonces que para nuestras muestras los átomos de carbono pueden quedar intersticiales o en los bordes de grano como resultado del molido. El producto resultante del molido es magnetoresistivo gigante, es decir que disminuye su resistividad eléctrica ante un campo magnético externo (a $77 \mathrm{~K}$ ). Por lo tanto debemos pensar que el Fe no se encuentra en un estado puro (como $\gamma$-Fe), al menos en parte, ya que tradicionalmente se acepta que esta fase del hierro es o bien antiferromagnética, con una temperatura máxima de Nèel de $67 \mathrm{~K}$, o bien no magnética. De acuerdo a cálculos teóricos [Fabricius 1994], el signo de la integral de intercambio $J$ en multicapas de hierro y cobre es muy sensible al parámetro de red. Pequeñas variaciones del parámetro hacen que el Fe sea FM, AFM o no magnético. El resultado del molido es, habíamos dicho, una solución fcc no ideal cuyo parámetro de red aumenta continuamente con la concentración. La existencia del efecto magnetoresistivo es posible sólo si tenemos entes magnéticos, con lo que podemos pensar que, a pesar del cambio en el parámetro de red con la concentración, no hay pérdida del carácter magnético del Fe. La medida de la resistividad pura nos da la pauta de que las muestras consisten en una mezcla donde es manifiesta la presencia de entes dispersantes. Las medidas de susceptibilidad nos muestran las características de un sistema mictomagnético, con un umbral de percolación en $x=15,9 \%$. Como los tamaños de grano que hemos medido en este sistema son muy pequeños (menores a 21 $\mathrm{nm}$ ), es muy factible que las aglomeraciones de hierro sean monodominio. Por tanto, es esperable un comportamiento superparamagnético. Entonces es importante ahora

${ }^{46}$ Ver Apéndice A 
preguntarnos si nuestro sistema es un superparamagneto o un mictomagneto. Debiéramos descartar que se trate de un superparamagneto no interactuante. Los intentos por ajustar la zona reversible de las curvas de $\mathrm{M}$ o $\chi$ vs $\mathrm{T}$, o de magnetización inicial vs $\mathrm{H}$ con una función de Langevin simple fueron infructuosos. Del cálculo de $\Theta$ vemos que la interacción entre partículas magnéticas es cada vez más intensa. Es decir que, en caso de ser superparamagnetos, son fuertemente interactuantes. Por otro lado, las diluciones de un metal de transición magnético en una matriz de metal noble han servido para fabricar sistemas mictomagnéticos. El cálculo del número $W$, propuesto en [Mydosh 1993], nos muestra que la relación es la encontrada comunmente en los vidrios de espín.

Que exista efecto magnetoresistivo gigante por encima de la temperatura crítica del sistema (en $x=10$ y 14,1) nos dice que los clusters no han perdido su magnetismo, con lo que vemos que la transición que hemos hecho es del tipo SG-SPM (ver el diagrama de fases de §I.3.4). No podemos decir nada respecto de las muestras más concentradas, ya que sólo las hemos podido medir magnetoresistividad a temperatura ambiente (además de a $\mathrm{T}=77 \mathrm{~K}$ ), donde tal vez la agitación térmica se imponga sobre la alineación magnética. De las medidas Mössbauer podemos decir que estos clusters parecen tener una concentración de Fe bastante constante, ya que a bajas temperaturas los espectros se parecen todos, con un campo magnético hiperfino promedio de $20 \mathrm{~T}$. Es de notar que todos los reportes coinciden en esto, sin importar el método de preparación ni la concentración (aún a concentraciones superiores al 50 \% de hierro, ver [Tie 1995]). El sistema transiciona a un estado paramagnético cuando pasa la temperatura crítica, y esto ocurre para todas las muestras.

Esos clusters, al aproximarse, favorecen el "anclado" de sus espines. Entonces, a temperatura constante, esto se manifiesta como una distribución de campos hiperfinos 
más ancha cuanto más aumenta la concentración de Fe. Esto es compatible con la idea de que tenemos un sistema de clusters superparamagnéticos interactuantes. Así, el hecho de que la manifestación del "anclado" recién aparezca a $\mathrm{x}=25 \%$ a temperatura ambiente se debe a que las interacciones intercluster son lo suficientemente intensas como para atenuar la agitación térmica, por lo que no es que exista otra percolación distinta, sino que se trata del mismo umbral de percolación magnética, pero visto a temperatura ambiente (el umbral calculado $\mathrm{C}_{\mathrm{p}}=15,9 \%$ corresponde a $\mathrm{T}=0 \mathrm{~K}$ ). Una confirmación adicional a la existencia de clusters de tamaño homogéneo y composición aproximadamente uniforme está dada por el comportamiento del desdoblamiento cuadrupolar, que permanece casi constante con una tendencia ligeramente creciente con $x$. Este valor es suficientemente alto, como ocurre en una región donde tenemos parte de los vecinos hierro y parte no. El aumento con la concentración proviene de un ligero enriquecimiento en Fe de los primeros vecinos de la sonda Mössbauer. Eso puede ocurrir tanto porque en el cluster hay un poco más de Fe como porque los clusters están más próximos entre sí, entonces, la sonda "siente" a los clusters vecinos. Las medidas Mössbauer de alta temperatura confirman estas conclusiones.

Por una vía diferente, el SAXS nos dice que el sistema es una colección de entes dispersantes de forma tridimensional, con interfases marcadas. Es evidente la existencia de una distribución de tamaños. Del ajuste a una colección de esferas extraemos que una suma de tres gaussianas es suficiente para describir esta distribución. Los tamaños típicos de las gaussianas son 2, 6 y $10 \mathrm{~nm}$. La contribución mayoritaria proviene de las “esferas" de $2 \mathrm{~nm}$. La contribución de los tamaños más grandes es mayor cuando la concentración aumenta. De todas maneras, el radio medio se mantiene aproximadamente constante. Del ajuste del invariante $Q$ a un sistema de dos fases 
estimamos que la concentración media de los entes dispersantes es del $35 \%$ atómico de hierro.

El efecto magnetoresistivo gigante en estas muestras es debido a la existencia de los clusters magnéticos. Estos clusters deben estar relacionados entre sí débilmente, así puede existir un "canal" de resistividad baja (de acuerdo a Fert). El efecto es mayor cuanto más fácilmente se alinean los clusters con el campo externo. Es decir, que el efecto es el producto de un juego entre tamaño y momento magnético de cada cluster, y de la separación con los clusters vecinos ${ }^{47}$. A muy bajas concentraciones de hierro, los clusters están lo suficientemente alejados unos de otros como para que la longitud de coherencia en los electrones de conducción sea menor, de lo que resulta que no hay efecto MRG. Al aumentar la concentración de hierro de la muestra, hay mayor densidad de clusters. De ahí, es posible entonces que se establezca un camino entre los clusters alineados con el campo externo. Es decir, la longitud de coherencia es del orden de las distancias intercluster, por lo que aparece el "canal" de resistividad baja para los espines. El aumento de la densidad de clusters hace que la interacción entre clusters cambie (a intercambio y/o dipolar), con lo que el "canal" se destruye, aunque no totalmente mientras existan clusters aislados. Así, a altas concentraciones (hasta $x=45$ $\%$ at., al menos) la MRG cae hasta casi desaparecer.

Podemos concluir que tenemos una dispersión de clusters magnéticos, de concentración $x=35 \%$ at. Fe, inmersos en una matriz conductora no magnética. El aleamiento mecánico produce estos clusters de manera bastante homogénea. El aumento en la concentración de hierro en los polvos primordiales conduce a la obtención de más clusters, pero de composición y tamaño similares (o sea, la composición y tamaño de los

\footnotetext{
${ }^{47}$ Otra fuente para la dispersión dependiente del espín es la rugosidad de la interfase [Levy 1994], la que supondremos similar en todas las muestras, ya que todas fueron preparadas de la misma manera.
} 
clusters son independientes de la cantidad de hierro original). Los clusters tienen mayoritariamente un tamaño de $2 \mathrm{~nm}$. El campo hiperfino de ellos es de aproximadamente $20 \mathrm{~T}$. El aumento en la cantidad de hierro primigenio da como resultado un aumento en la densidad de clusters magnéticos, los que a partir de una concentración $\left(\mathrm{C}_{\mathrm{p}}=15,9 \%\right)$ empiezan a percolar magnéticamente y luego espacialmente. La magnetoresistividad gigante es una consecuencia de la densidad de clusters en la muestra. 


\section{Capítulo II}

\section{El sistema Fe-Cu tratado térmicamente}

1. Introducción

2. Preparación de las muestras

3. Medidas

1. Magnetoresistividad

2. Resistividad y magnetoresistividad

3. Difracción de rayos $X$

4. Medidas magnéticas

5. Espectroscopía Mössbauer

\section{Discusión}




\section{Introducción}

Una vez producidas las muestras $\mathrm{Fe}_{\mathrm{x}} \mathrm{Cu}_{100-\mathrm{x}}$ en el rango donde se presentaba el efecto magnetoresistivo gigante buscamos un tratamiento térmico que mejorase dicho efecto. La idea original era ver si se podía lograr un mayor efecto absoluto $\Delta \rho$ y/o una mejora en la resistividad de la red $\rho$. Como se contó en §I.1 "Trabajos previos", mucho de la investigación sobre el sistema $\mathrm{Fe}-\mathrm{Cu}$ se focalizó en la composición equiatómica y el proceso de su descomposición con la temperatura. Algo de la investigación se hizo en otras composiciones [Saxena 1994, Eilon 1995, Yermakov 1995, Ueda 1996] donde se han reportado resultados del efecto del tratamiento térmico sobre la magnetoresitividad gigante.

\section{Preparación de las muestras}

Preparamos las muestras de la siguiente manera: tomamos las muestras compactadas sobre las cuales habíamos medido magnetoresistividad, y las cerramos en ampollas de vidrio o cuarzo en atmósfera de argón e hidrógeno (en proporción 2:1), a baja presión (0,3 atm). Colocamos las ampollas en hornos eléctricos con control de rampa. Los hornos utilizados fueron todos del mismo tamaño. En todos los casos llevamos el horno hasta la temperatura deseada, y una vez alcanzada la misma colocábamos la ampolla. El enfriamiento del horno producido por la introducción de la ampolla era compensado rápidamente por el control electrónico del mismo. Al finalizar el tiempo deseado quitábamos la ampolla del horno y la dejábamos enfriar al aire. Probamos diversos regímenes de tratamiento térmico, variando la temperatura y el tiempo de tratamiento térmico en busca del mejoramiento del efecto magnetoresistivo gigante $\Delta \rho / \rho$.

Encontramos que para el sistema Fe-Cu la temperatura óptima es de $300 \pm 50^{\circ} \mathrm{C}$. El tiempo óptimo de tratamiento para esta temperatura es de aproximadamente 24 horas. 
Para la realización de la difracción de rayos X y las medidas Mössbauer pulverizamos los lingotes que habíamos tratado térmicamente.

\section{Medidas}

\subsection{Magnetoresistividad}

Como vemos en la figura 1 , las muestras tratadas a $300{ }^{\circ} \mathrm{C}$ durante 24 horas

aumentaron el
efecto
magnetoresistivo,
comparadas con los
de las muestras sin
tratar.
comportamiento con
la concentración es
similar a las sin
tratar (las muestras

el

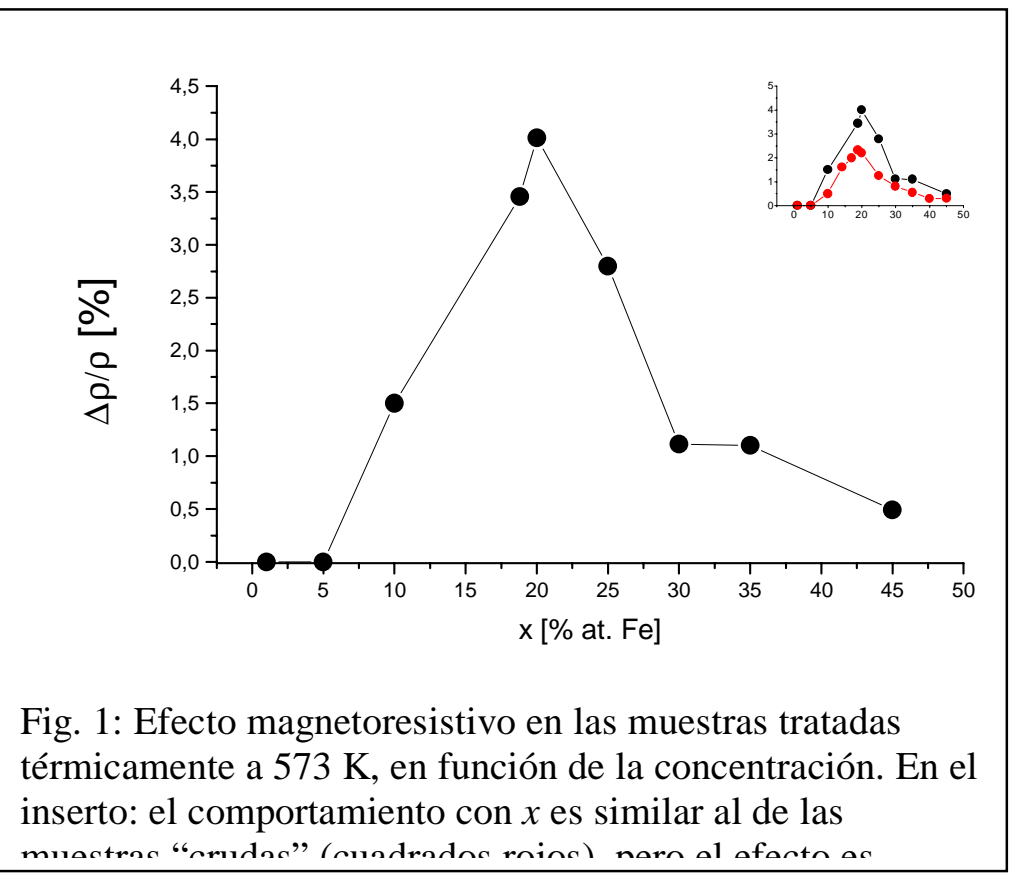

“crudas"), es decir, el efecto aumenta a $4 \%$ en $x=20 \%$, para luego caer hasta hacerse muy pequeño a $45 \%$. Estos resultados son algo distintos a los de [Ueda 1996], donde la temperatura del tratamiento térmico hace bajar la concentración a la que se alcanza el máximo en la razón magnetoresistiva, desde $x=30 \%$ at. Fe hasta $28 \%$ (para la muestra tratada a $523 \mathrm{~K})$ y a $25 \%$ at. (para la muestra tratada a 573 K). Según estos autores, ese cambio se debe a que el tratamiento modifica el tamaño de las
Fig. 2: Efecto magnetoresistivo en $\mathrm{Fe}_{20} \mathrm{Cu}_{80}$ molida por 75 horas y tratada térmicamente a $573 \mathrm{~K}$ durante 24 horas. 
partículas llevándolas al tamaño adecuado para dar el máximo efecto MRG. La figura 2 muestra una de las medidas de magnetoresistividad tomada en estas muestras tratadas térmicamente. Los puntos aparecen algo dispersos, en comparación con los de sus equivalentes crudas. Esta característica se repite en todas las muestras sometidas al tratamiento térmico antes descripto. En uno de los casos donde se efectuó un tratamiento térmico más prolongado (48 horas), la dispersión de puntos disminuyó notablemente, y el efecto MRG se mantuvo.

\subsection{Resistividad y magnetoresistividad}

La medida de resistividad pura $\rho$ en estas muestras es diferente a la de las muestras crudas. Vemos que la resistividad es sustancialmente menor para estas muestras, como es esperable que ocurra luego de un tratamiento térmico

prolongado que ha eliminado defectos en la red. Además, ya el sistema no sigue la regla de Nordheim como antes; más bien, presenta sus valores más altos en la zona alrededor de $x=20 \%$. Como veremos en $\$ I I .3 .5$, el tratamiento térmico favorece la precipitación de otros compuestos, como carburos y

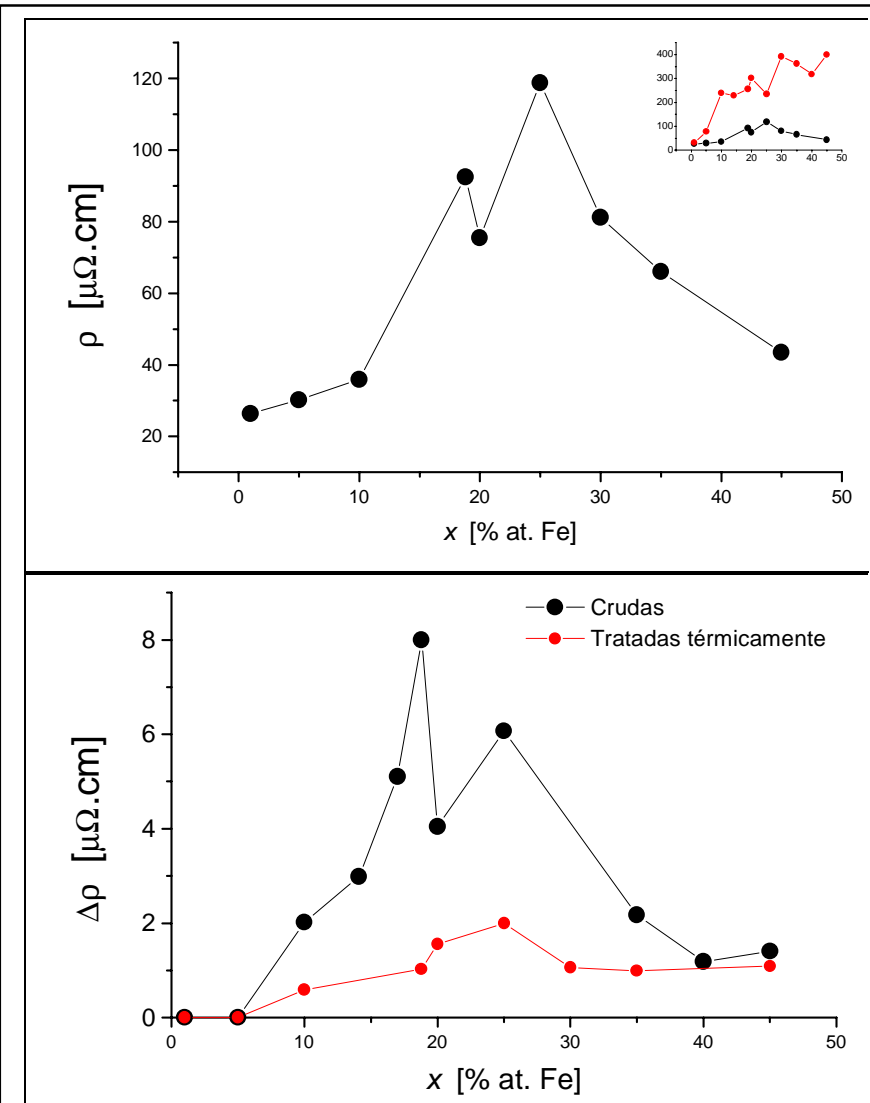

Fig. 3: arriba) Resistividad pura de las muestras tratadas térmicamente. abajo) Magnetoresistividad $\Delta \rho$ medida en las muestras tratadas térmicamente (en rojo) y en las crudas (en negro)

óxidos, los que hacen que el sistema no se pueda describir simplemente como una 
mezcla de un soluto y un solvente. Hay un orden de magnitud de diferencia entre nuestros valores y los de la resistividad de los metales originales. En la parte inferior de la figura 3 vemos que la magnetoresistividad $\Delta \rho$ es menor en las tratadas térmicamente que en las muestras crudas, lo que puede deberse a un engrosamiento de los clusters magnéticos, a una disminución en la rugosidad de las interfases de los clusters, o a la presencia de más interacciones magnéticas, originadas en las nuevas especies precipitadas. Es interesante que notemos que Xiao et al ([Wang 1994]) midieron $\Delta \rho=$ 2,3 $\mu \Omega \mathrm{cm}$ a 4,2 $\mathrm{K}$ y $1,4 \mathrm{~T}$ en una muestra $\mathrm{Fe}_{20} \mathrm{Cu}_{80}$ preparada por cosputtering.

La mejora en la razón magnetoresistiva $\Delta \rho / \rho$ se debe a que la mejora en la resistividad pura $\rho$ es muy notable, de más del $50 \%$ en algunos casos, y como es más grande en las crudas que en las muestras tratadas térmicamente, al hacer el cociente tenemos una cantidad mayor.

El decrecimiento en $\Delta \rho$ como consecuencia del tratamiento térmico fue observado en [Wang 1994] y en el sistema Co-Cu preparado por MA ([Yasuna A]).

\subsection{Difracción de rayos $X$}

Hicimos DRX con los mismos aparatos y en las mismas condiciones que con las muestras "crudas". El aspecto general de los difractogramas es el mismo que el correspondiente al de las muestras crudas, excepto que en varios de ellos es distinguible un pico, localizado a $2 \theta \approx 44,7^{\circ}$ (fig. 4). El componente que presenta una reflexión intensa más próxima es el $\alpha$-Fe, ya que su pico (110) se debiera ver a 44,671 ${ }^{\circ}$ La importancia de este pico aumenta con la concentración de una forma bastante sistemática, lo cual nos da la pauta que se trata de la reflexión del $\alpha$-Fe antes mencionada.

El parámetro de red de la parte fcc de la muestra fue calculado excluyendo al pico (111), dado que ocurría lo mismo que en las muestras crudas. El parámetro de red 
es siempre mayor que el correspondiente al cobre puro (ver figura 5), pero siempre menor al de las muestras crudas (inserto de la misma figura), y no evidencia dependencia con la composición. El parámetro es siempre mayor a lo que indica la Ley de Vegard, tomando como extremo del hierro puro al parámetro de red del $\gamma$-Fe $(a=$ 0,36467 nm, [CRC 2000]). Calculamos el parámetro de red del bcc-Fe mediante la

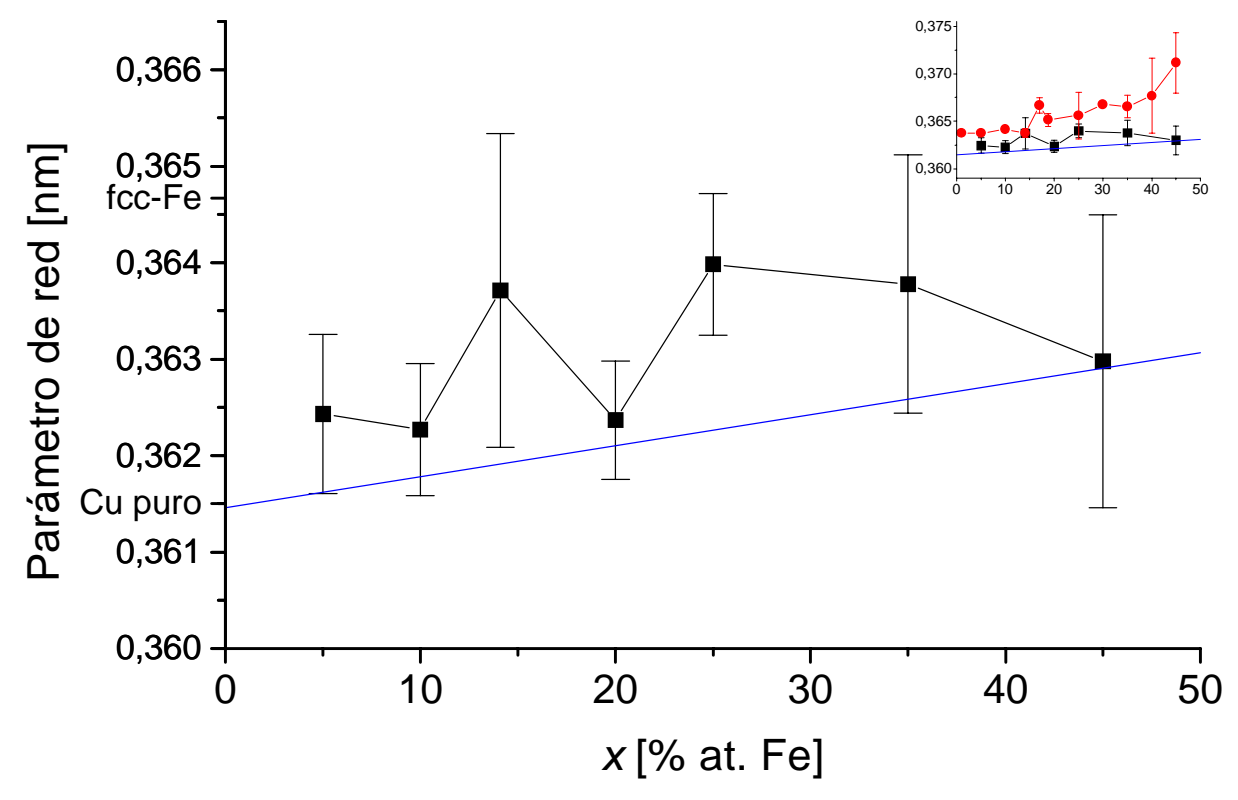

Fig. 5: Parámetro de red de las muestras tratadas térmicamente vs concentración de Fe. En el inserto se compara con las medidas sobre las muestras "crudas" (en rojo). En ambos gráficos la línea recta azul representa la ley de Vegard tomando al fcc-Fe como unn de los extremns

medida de la posición angular $\theta$ del pico (110). Para ello colocamos esta medida en:

$$
a=\lambda \sqrt{h^{2}+k^{2}+l^{2}} / 2 \operatorname{sen} \theta,
$$

con $h=1, k=1, l=0, \mathrm{y} \lambda$ la longitud de onda del rayo $\mathrm{K}_{\alpha 1}$ del cobre. El resultado fue que el parámetro de red del $\alpha$-Fe se mantiene constante con $x$ (fig. 6), siendo su valor de $0,2867 \pm 0,0039 \mathrm{~nm}$, en excelente acuerdo con el valor aceptado para el $\alpha$-Fe ([CRC 2000]). 
En [Ueda 1996] se reporta también la disminución del parámetro de red de la estructura fcc luego de aplicar un tratamiento térmico a 523 K. Éste permanece por encima del parámetro del cobre puro, para todas las muestras entre 20 y $60 \%$ at. En [Macrí 1994] se reporta el mismo comportamiento para el $\mathrm{Fe}_{50} \mathrm{Cu}_{50}$

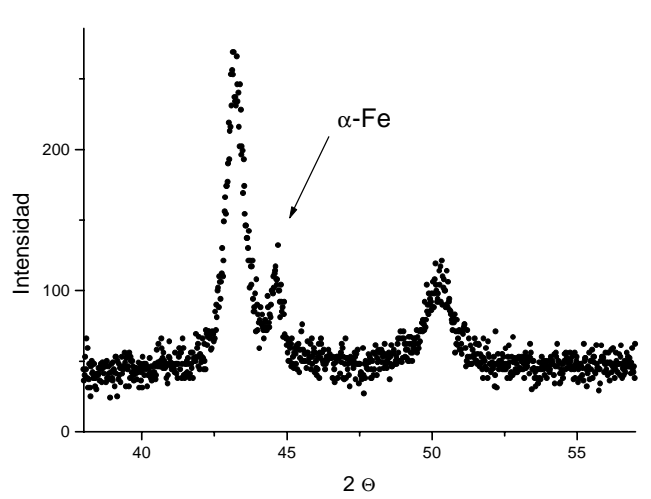

Fig. 4: Sector del difractograma tomado a la muestra de $\mathrm{Fe}_{35} \mathrm{Cu}_{65}$ tratada térmicamente, donde aparece el pico

tratado a $573 \mathrm{~K}$. En ninguno de ambos se menciona al pico del bcc-Fe que hemos encontrado, si bien en esta última referencia se ve en la figura 7 un esbozo de pico en esa posición. Para Eckert et al ([Eckert 1993]) hay un pequeño descenso en $a$ con el tratamiento a $523 \mathrm{~K}$, pero no reportan la existencia del pico bcc hasta una temperatura muy superior. Eilon et al ([Eilon 1995]) reportan que ya a $473 \mathrm{~K}$ hay precipitación de $\alpha-\mathrm{Fe}$, y que el parámetro de red de la aleación fcc decrece. Es probable que las diferencias

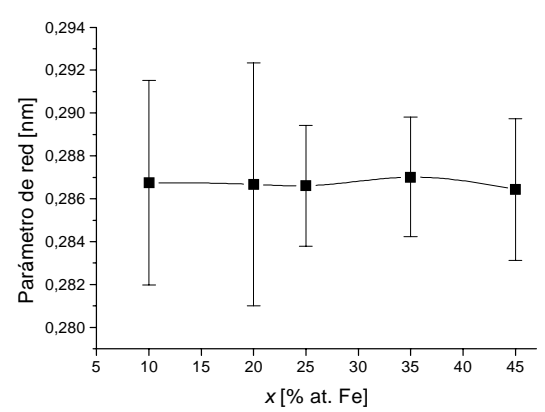

Fig. 6: Parámetro de red del hierro nrecinitadn

con nuestros resultados se deban a que nuestro tratamiento térmico es mucho más prolongado (24 horas contra tiempos del orden de una hora). La diferencia con el trabajo de Eilon también puede deberse a que en su muestra hay una pequeña cantidad remanente de $\alpha$-Fe, visible por EM.

\section{$\underline{\text { Tamaños de grano }}$}


El tamaño de los granos fcc Fe-Cu se mantiene en una franja entre los 15 y 30 $\mathrm{nm}^{1}$, sin dependencia con la concentración. El cálculo de los tamaños de la fase fcc lo hicimos de manera descripta en §I.3.2. Ocurre algo similar con el tamaño de grano del pico atribuído al $\alpha-\mathrm{Fe}$, que oscila entre 4 y $35 \mathrm{~nm}$ (figura 7). El tamaño de grano de la fase bcc fue calculado en base a la fórmula de Scherrer²:

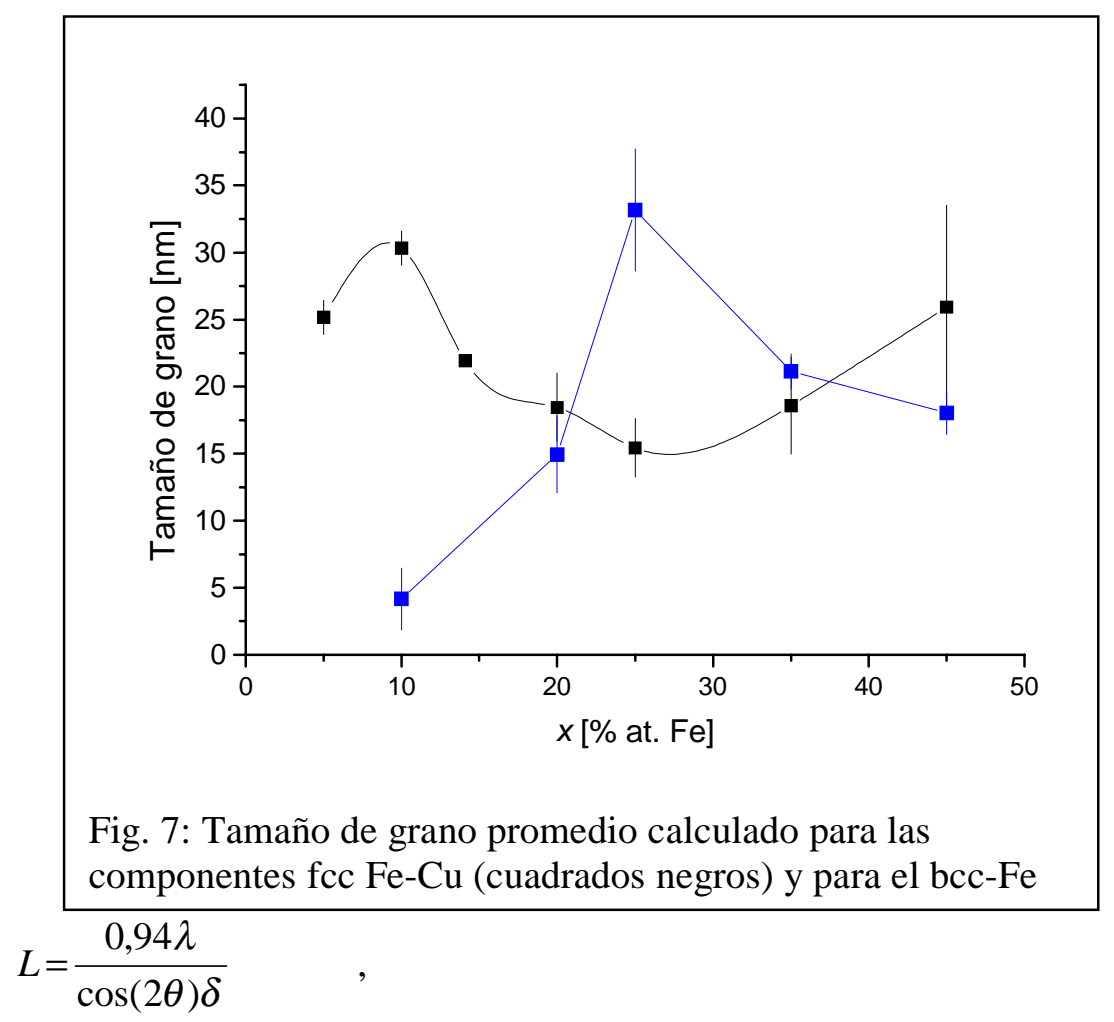

con el ancho total a mitad de altura del pico $\delta$ medido en radianes, $2 \theta$ la posicion angular del pico (en la escala $2 \theta$ ) y $\lambda$ la longitud de onda del rayo $K_{\alpha l}$ del cobre. Este cálculo no es muy preciso, pero es útil para calcular un tamaño medio de las partículas. Estos resultados nos están diciendo que los tamaños medios de ambas fases son comparables, y no cambian debido a la mayor o menor abundancia de hierro.

\footnotetext{
${ }^{1}$ Comparable con los $\approx 13 \mathrm{~nm}$ reportados en [Eckert 1993]

2 Tomada de [Warren 1990]
} 


\subsection{Medidas magnéticas}

Las medidas de susceptibilidad las hicimos algunas en modo de rampa y otras en modo de lista (ver apéndice C) con el susceptómetro Lakeshore 7130 ya mencionado en el capítulo anterior. La figura 8 muestra los resultados. Las medidas de concentración $x$ $\leq 18,8 \%$ muestran que la susceptibilidad aumenta con la temperatura, como ocurriría en

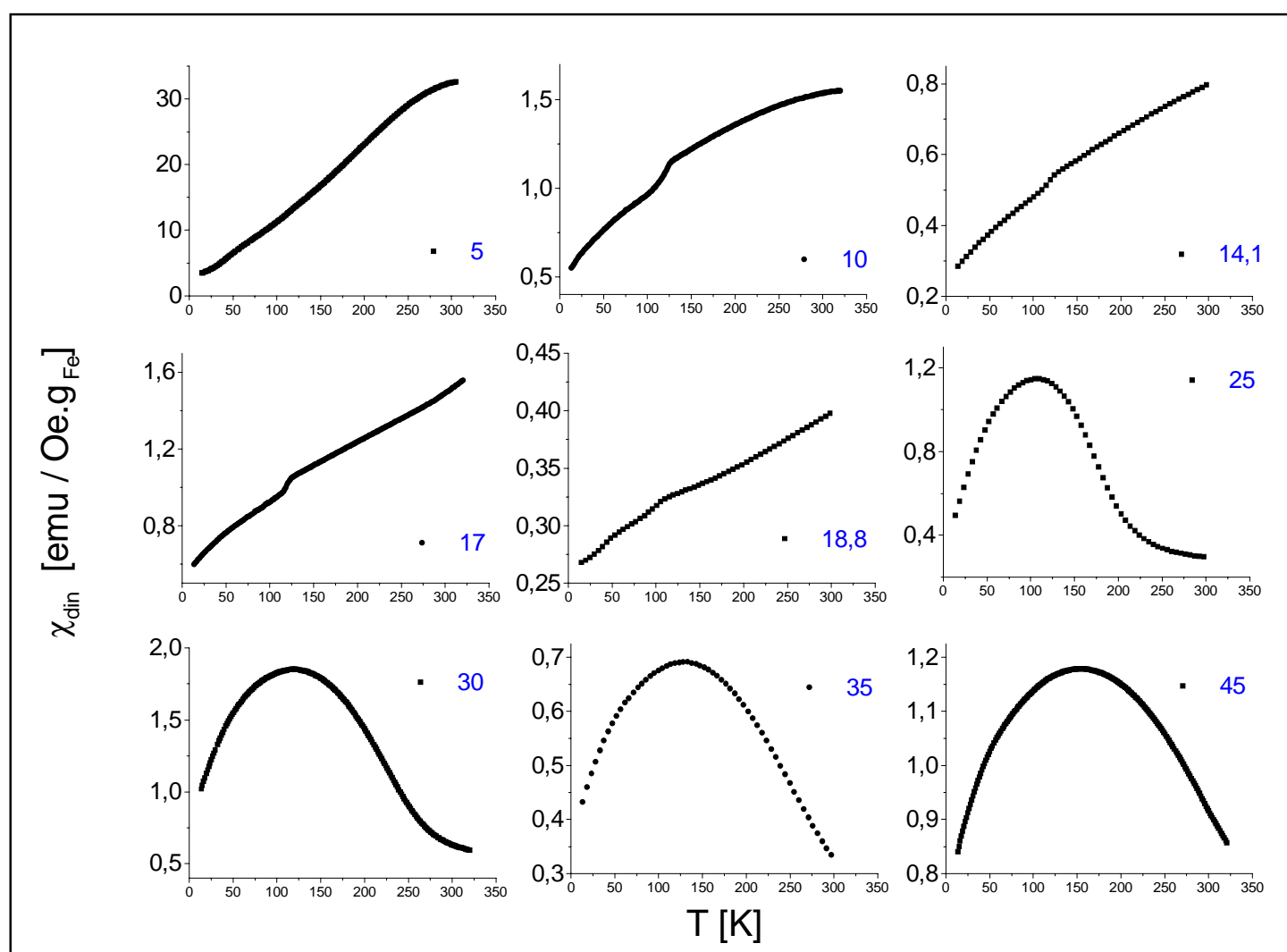

Fig. 8: Susceptibilidad dinámica $\chi$ ' medida con un campo director de 1 Oe y frecuencia $\omega$ $=375 \mathrm{~Hz}$. En azul indicamos las concentraciones. La disparidad en los valores de la suscentihildad nueden estar orioinados en los modos en oue efectuamos las mediciones un ferromagneto. Es posible observar un "promontorio" para $\mathrm{T} \approx 115-127 \mathrm{~K}$. Puede estar ocurriendo que un pico tipo SG-SPM esté cubierto por una componente ferromagnética. La muestra con $x=5 \%$ presenta valores muy altos, por lo que la excluiremos de nuestros análisis. Para $x=25 \%$ y las concentraciones superiores vemos un pico redondeado que se desplaza hacia mayores temperaturas con el aumento de $x$. Hemos colocado estos puntos en el diagrama de fases obtenido en el capítulo I. Como 
vemos en la figura 9 las temperaturas del promontorio caen con la concentración. Cuando $x \geq 25$, las temperaturas del máximo crecen ligeramente con la concentración.

Si comparamos muestra a muestra utilizando las figuras 8 de la página anterior y 10 del capítulo anterior, la muestra tratada térmicamente tiene una mayor respuesta magnética que su correspondiente cruda.

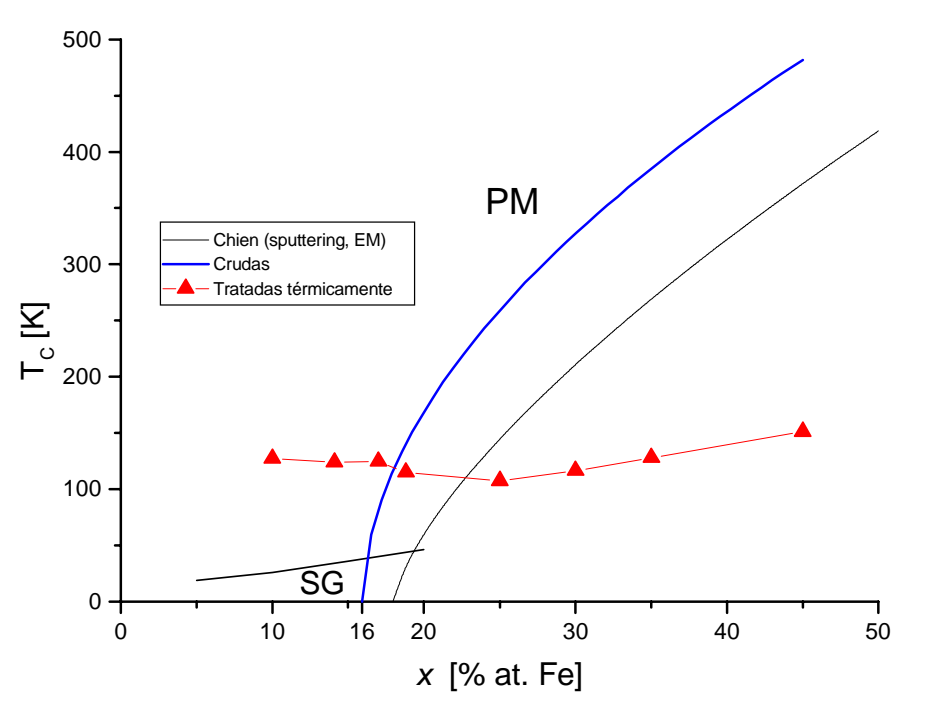

Fig. 9: Temperaturas de promontorio $(\mathrm{x} \leq 18,8)$ y de máximo $(x \geq 25)$ sobre el diagrama de fases obtenido en el capítulo

\subsection{Espectroscopía Mössbauer}

Medimos por espectroscopía Mössbauer a las muestras tratadas térmicamente. Los espectros (ver fig. 10) se componen de varios subespectros, de los cuales entre tres y cinco (dependiendo de qué muestra se trate) son sextetes, más un doblete y un singlete. En la figura 11 se muestra el espectro de $\mathrm{Fe}_{40} \mathrm{Cu}_{60}$ tratado térmicamente con su ajuste completo. En las tablas I y II se indican los parámetros hiperfinos de todas las muestras medidas. Del análisis de éstos podemos adscribir uno de los sextetes al $\alpha$-Fe, dos de los otros sextetes a algún carburo de hierro similar a la cementita o al carburo de 
$\mathrm{Häag}^{3}$, y los otros dos restantes a óxidos de Fe u óxidos mixtos de $\mathrm{Fe}$ y $\mathrm{Cu}$. Hemos asignado el singlete al $\gamma$ - $\mathrm{Fe}^{4}$, cuya precipitación con los tratamientos térmicos ha sido reportada en varios trabajos [Macrí 1994, Drbohlav 1994, Ding 1995]. Finalmente, consideramos al doblete (quien tiene parámetros hiperfinos similares a los de las muestras sin tratar) como proveniente de la primigenia solución fcc $\mathrm{Fe}-\mathrm{Cu}$. Como vemos en la figura 12, el área relativa de los subespectros (hemos sumado las

${ }^{3}$ Ambos carburos poseen estequiometría similar: la cementita es $\mathrm{Fe}_{3} \mathrm{C}$ y el carburo de Häag $\mathrm{Fe}_{2,5} \mathrm{C}$. Estos carburos son ortorrómbico y monoclínico, respectivamente, con parámetros de red grandes, aunque las distancias entre hierros $(0,26 \mathrm{~nm})$ son bastante similares al parámetro de red del bcc-Fe.

${ }^{4}$ Su corrimiento isomérico es un poco más negativo que lo aceptado. Pensamos que esto se debe en parte a un efecto de compresión sobre el sitio. De acuerdo al cálculo efectuado por Ingalls (véase el artículo de ¿??, en [Shenoy 1978]), se estima que el cambio en el corrimiento isomérico $\delta$ debido al cambio fraccional en el volumen, para el hcp-Fe, es $\frac{\partial \delta}{\partial \ln \Omega}=\alpha=0,84$. La diferencia entre el $\delta$ medido $(-0,14 \mathrm{~mm} / \mathrm{s}$ en promedio) y el aceptado para el $\gamma$-Fe $(-0,01$ $\mathrm{mm} / \mathrm{s}$ ) es de $-0,04 \mathrm{~mm} / \mathrm{s}$, con lo que el cambio fraccional es $\Delta \Omega / \Omega_{0}=-0,048 \Rightarrow$ $\frac{\Omega-\Omega_{0}}{\Omega_{0}}=\frac{\Omega}{\Omega_{0}}-1=-0,048$, donde $\Omega_{0}$ es el volumen no sometido a compresión. Seguimos que

$\frac{\Omega}{\Omega_{0}}=1-0,048=0,952$, y como $\frac{\Omega}{\Omega_{0}}=\left(\frac{a}{a_{0}}\right)^{1 / 3}=0,952^{1 / 3}=0,98$, con $a_{0}$ el parámetro de red del sistema no comprimido. Esto siginifica que la disminución del corrimiento isomérico observada correspondería a una disminución del parámetro de red del $2 \%$, comparado con el valor aceptado para el $\gamma$-Fe $(0,36467 \mathrm{~nm})$. A pesar que la disminución promedio del parámetro de red a es de 0,4\%, el cálculo da la tendencia correcta. 
contribuciones de los óxidos en una sola ${ }^{5}$, y las del carburo ${ }^{6}$ en otra) cambia con la concentración. La contribución del doblete disminuye sensiblemente con el aumento de la cantidad de hierro. Cae desde un valor máximo de $62 \%$ (en $x=14,1 \%$ ) a $18,8 \%$ (en $x=45 \%)$. En cambio, el carburo da cuenta de alrededor de un $30 \%$ del espectro en las muestras con $x \geq 18,8 \%$. Los óxidos no aparecen en todas las muestras, pero a pesar de eso el diagrama sugiere que su abundancia aumenta con la concentración. Al no

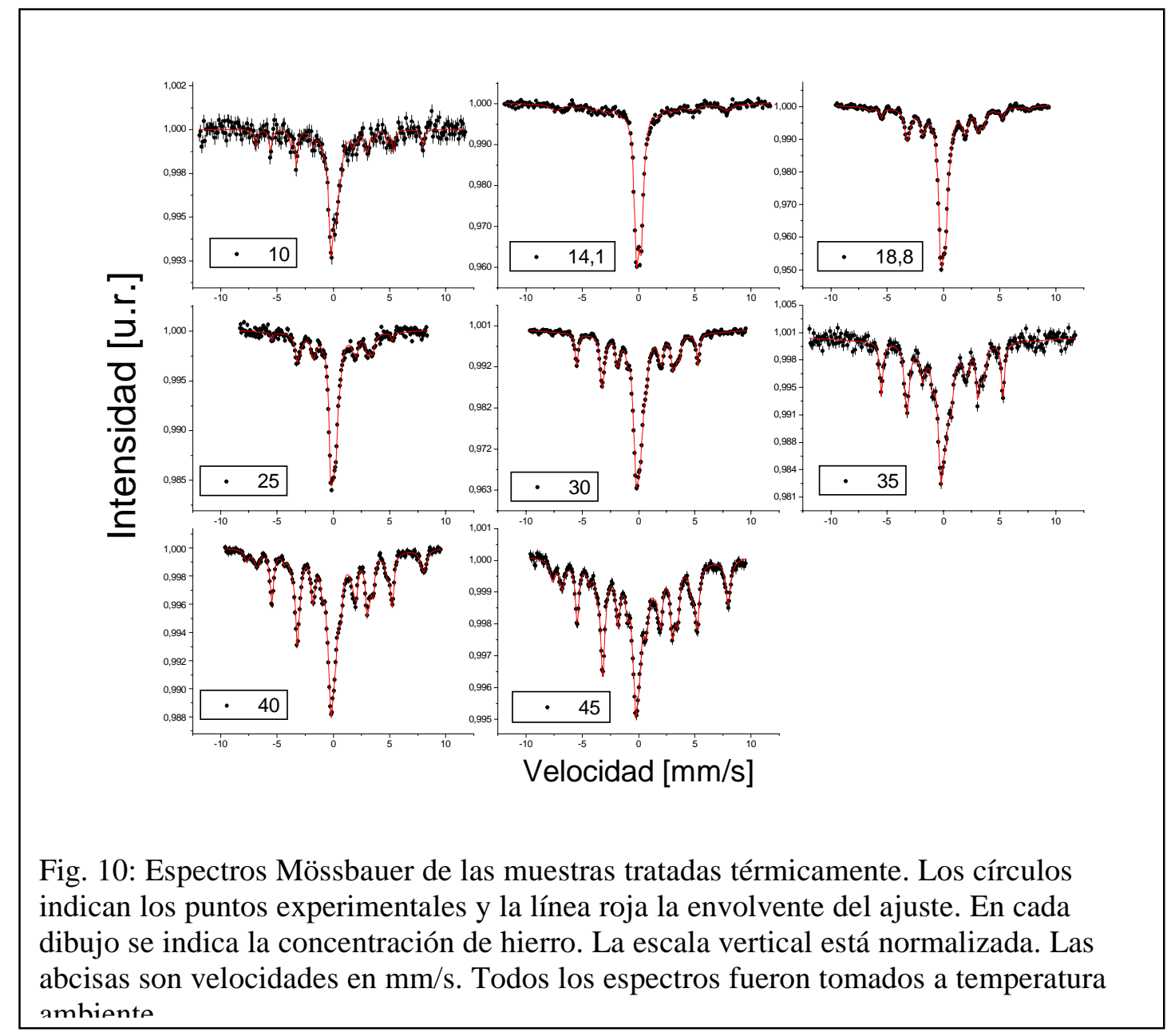

aparecer sistemáticamente hemos decido que se trata de un contaminante casual de

\footnotetext{
${ }^{5}$ El resultado del ajuste nos permite adscribir los dos sextetes a la magnetita $\left(\mathrm{Fe}_{3} \mathrm{O}_{4}\right)$, que posee dos sitios, con $B_{\mathrm{hf}}=49$ y $46 \mathrm{~T}$ y $\delta=0,26$ y $0,67 \mathrm{~mm} / \mathrm{s}$, y es ferrimagnética hasta $850 \mathrm{~K}$. Datos tomados de [Bowen 1993]. En el caso de las muestras con $x=10$ y 18,8 sólo hemos podido ajustar un sextete de $B_{h f}$ alto, tal vez debido a la poca área relativa de este sitio. ${ }^{6}$ El carburo de Häag tiene dos sitios distintos en una relación 2:1, con campos un tanto diferentes $\left(B_{h f}=18,5 \pm 0,2 \mathrm{~T}\right.$ y $\delta=0,17-0,2 \mathrm{~mm} / \mathrm{s}$ para el sitio I; y 21,6 $\pm 0,2 \mathrm{~T}$ y $\delta=0,23 \mathrm{~mm} / \mathrm{s}$ para el sitio II). La cementita tiene un campo de $20,7 \mathrm{~T}$ y $\delta=0,18 \mathrm{~mm} / \mathrm{s}$ (promedio de ambos sitios) a temperatura ambiente. Datos tomados de [Le Caër 1976].
} 
algunas de las muestras. Esto se ve reforzado por tratarse de magnetita, quien se forma en ambientes pobres en oxígeno. El $\gamma$-Fe no pasa de un $12 \%$ del espectro como máximo (en la muestra con $x=25 \%$ ), y va disminuyendo levemente con el aumento de la concentración de hierro. El $\alpha$-Fe va creciendo en importancia, hasta llegar a ser un $21 \%$ del espectro (en $x=40 \%$ ). Es decir, que con el aumento de la concentración, los sitios de la aleación fcc Fe-Cu se despueblan en favor del carburo, el hierro bcc y los óxidos. El $\gamma$-Fe disminuye su participación hasta desaparecer para $x=45 \%$. 


\begin{tabular}{|c|c|c|c|c|c|c|c|c|c|c|}
\hline Sitio & \multicolumn{2}{|c|}{$\alpha-\mathrm{Fe}$} & \multicolumn{2}{|c|}{ Carburo A } & \multicolumn{2}{|c|}{ Carburo B } & \multicolumn{2}{|c|}{ Óxido 1} & \multicolumn{2}{|c|}{ Óxido 2} \\
\hline$x$ & $\mathrm{~B}_{\mathrm{hf}}$ & $\delta$ & $\mathrm{B}_{\mathrm{hf}}$ & $\delta$ & $\mathrm{B}_{\mathrm{hf}}$ & $\delta$ & $\mathrm{B}_{\mathrm{hf}}$ & $\delta$ & $\mathrm{B}_{\mathrm{hf}}$ & $\delta$ \\
\hline 10 & $339,712_{1,576}$ & $-0,003_{0,022}$ & $189,63_{5,825}$ & $0,025_{0,068}$ & $213,115_{4,754}$ & $0,242_{0,57}$ & - & - & $460,528_{1,941}$ & $0,636_{0,025}$ \\
\hline 14,1 & $329,981_{0}$ & $0,0_{0}$ & $167,32_{3,788}$ & $0,241_{0,05}$ & $202,03_{4,756}$ & $0,249_{0,061}$ & - & - & - & - \\
\hline 18,8 & $334,387_{1,073}$ & $0,002_{0,015}$ & $191,983_{1,646}$ & $0,165_{0,013}$ & $212,473_{1,15}$ & $0,228_{0,012}$ & - & - & $441,121_{2,963}$ & $0,706_{0,041}$ \\
\hline 25 & $331,649_{1,838}$ & $-0,009_{0,027}$ & $185,644_{2,624}$ & $0,18_{0,021}$ & $208,772_{1,69}$ & $0,22_{0,014}$ & - & - & - & - \\
\hline 30 & $334,606_{0,301}$ & $-0,006_{0,004}$ & $197,95_{0,909}$ & $0,188_{0,007}$ & $217,027_{0,752}$ & $0,221_{0,007}$ & - & - & - & - \\
\hline 35 & $336,277_{0,518}$ & $0,007_{0,007}$ & $203,267_{2,593}$ & $0,209_{0,023}$ & $220,102_{2,357}$ & $0,244_{0,027}$ & - & - & - & - \\
\hline 40 & $332,154_{0,344}$ & $-0,006_{0,005}$ & $193,843_{1,078}$ & $0,173_{0,01}$ & $210,887_{0,633}$ & $0,233_{0,007}$ & $491,41_{1,979}$ & $0,304_{0,026}$ & $457,943_{1,553}$ & $0,677_{0,022}$ \\
\hline 45 & $331,831_{0,359}$ & $-0,002_{0,005}$ & $195,73_{1,363}$ & $0,164_{0,013}$ & $207,736_{0,719}$ & $0,225_{0,009}$ & $485,04_{1,995}$ & $0,258_{0,023}$ & $458,508_{1,624}$ & $0,704_{0,019}$ \\
\hline
\end{tabular}

Tabla I: Campos magnéticos hiperfinos $\left(\mathrm{B}_{\mathrm{hf}}\right)$ y sus corrimientos isoméricos $(\boldsymbol{\delta})$ obtenidos. En subíndice el error correspondiente. El valor cero indica que se fijó el valor para el ajuste 


\begin{tabular}{|c|c|c|c|}
\hline \multirow{2}{*}{ Concentración } & \multicolumn{2}{|c|}{ Solución sólida $\mathrm{Fe}-\mathrm{Cu}$} & \multirow{2}{*}{$\begin{array}{c}\gamma-\mathrm{Fe} \\
\delta[\mathrm{mm} / \mathrm{s}]\end{array}$} \\
\hline & $\Delta[\mathrm{mm} / \mathrm{s}]$ & $\delta[\mathrm{mm} / \mathrm{s}]$ & \\
\hline 10 & $0,396_{0,083}$ & $0,204_{0,059}$ & $-0,13_{0,019}$ \\
\hline 14,1 & $0,406_{0,01}$ & $0,151_{0,009}$ & $-0,164_{0,03}$ \\
\hline 18,8 & $0,326_{0,012}$ & $0,143_{0,011}$ & $-0,167_{0,006}$ \\
\hline 25 & $0,281_{0,037}$ & $0,2_{0,034}$ & $-0,128_{0,013}$ \\
\hline 30 & $0,296_{0,024}$ & $0,137_{0,016}$ & $-0,141_{0,007}$ \\
\hline 35 & $0,296_{0}$ & $0,125_{0,028}$ & $-0,133_{0,018}$ \\
\hline 40 & $0,292_{0,03}$ & $0,1_{0,026}$ & $-0,119_{0,016}$ \\
\hline 45 & $0,201_{0,113}$ & $0,073_{0,016}$ & $-0,153_{0,011}$ \\
\hline
\end{tabular}

Tabla II : Parámetros hiperfinos del doblete y el singlete. En subíndice el error correspondiente. 


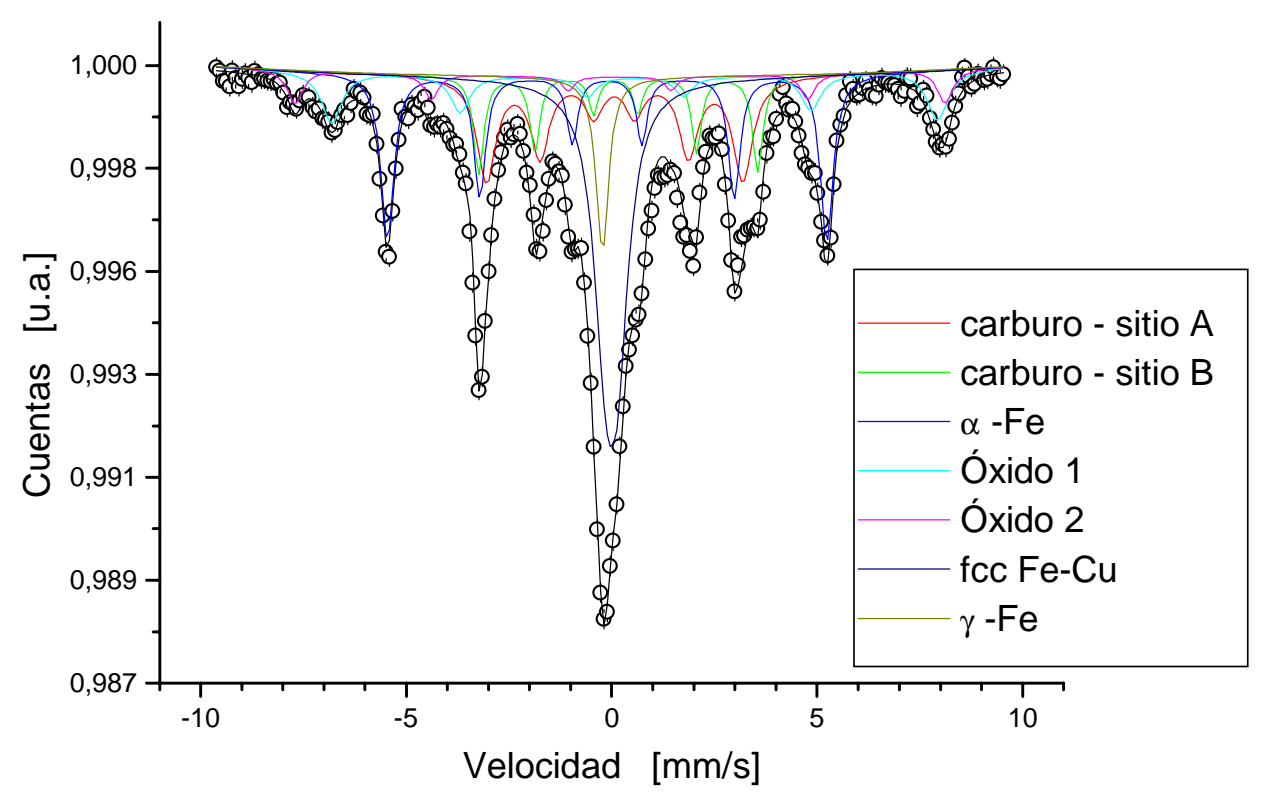

Fig. 11: Espectro Mössbauer con el ajuste completo de $\mathrm{Fe}_{40} \mathrm{Cu}_{60}$ tratado térmicamente. La línea negra indica la envolvente. Las otras líneas corresponden a los distintos subespectros ajustados.

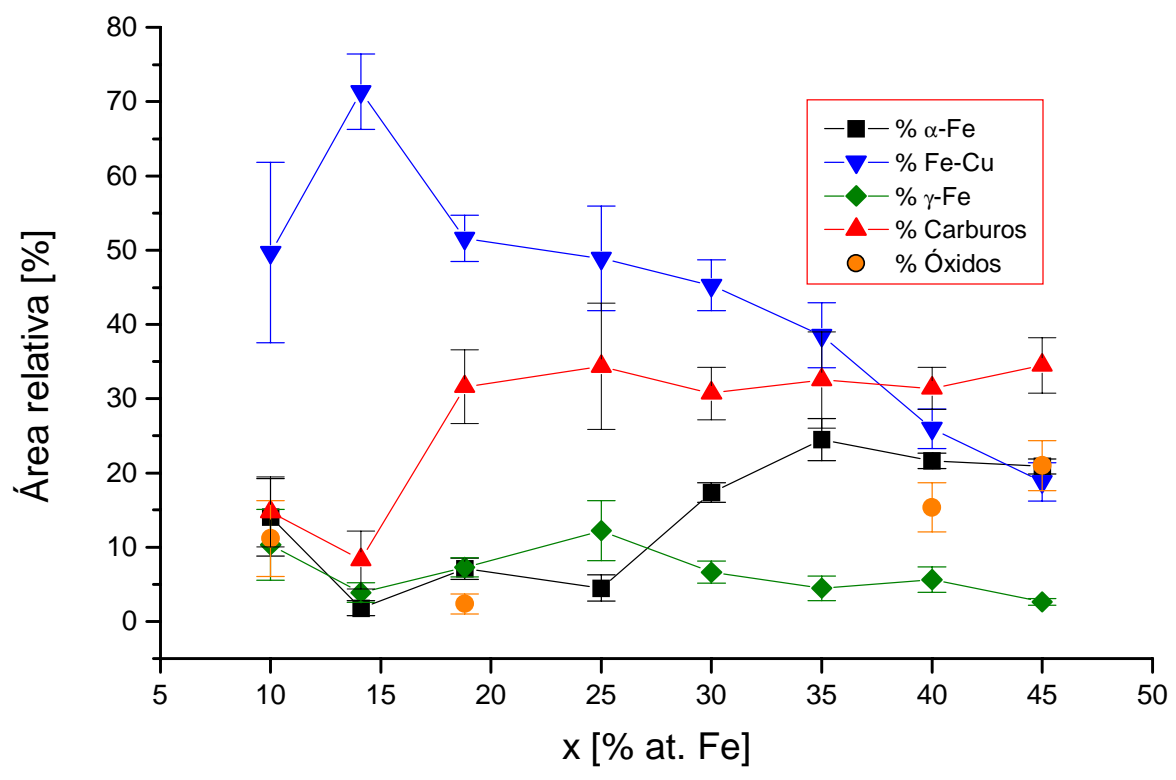

Fig. 12: Áreas relativas vs. concentración de hierro. Los porcentajes correspondientes a los dos subespectros de los carburos están sumados, así como 


\section{Discusión}

El tratamiento térmico a $300{ }^{\circ} \mathrm{C}$ durante 24 horas provee la energía térmica que favorece la difusión de los elementos. Esto produce una relajación de los defectos, como confirman las medidas de resistividad pura. También se produce la migración del hierro y el carbono. Sabemos que debido al calor positivo de mezcla del hierro y el cobre, la aleación obtenida es metaestable. Tanto nuestros resultados como la literatura existente dan cuenta de que por encima de $300{ }^{\circ} \mathrm{C}$ comienza la destrucción de la aleación $\mathrm{Fe}-\mathrm{Cu}$, es decir que la segregación de fases empieza a ser evidente. Si aumentamos la temperatura, el sistema se segrega casi por completo en $\mathrm{Cu}$ y $\alpha$-Fe macroscópico. El camino de los electrones de conducción ya no tendría suficientes entes dispersores como para que el efecto magnetoresistivo gigante aparezca o sea relevante. Esto lo hemos visto reflejado en la magnetoresistividad, ya que las muestras tratadas por encima de los $350{ }^{\circ} \mathrm{C}$ no presentan MRG.

La aparición de un carburo del hierro es evidente en los espectros Mössbauer, y da cuenta de un $30 \%$ de los átomos de Fe. La solubilidad del carbono en el cobre es muy baja $(0,04 \%$, [Massalski 1990]), por lo que al hacer el tratamiento térmico se facilita la expulsión del C hacia los bordes de grano o hacia las interfases con los clusters ricos en hierro. Los átomos de carbono que migran hacia las interfases formarán carburos con los átomos de hierro de esos clusters. La solubilidad del $\mathrm{C}$ en Fe también es baja, pero al existir compuestos metaestables en las zonas ricas en hierro pueden producirse carburos. También los carbonos ubicados en los bordes de grano de la solución sólida fcc pueden formar carburos con los átomos de hierro de esos bordes. De cualquier manera, no se encuentran en tamaños suficientemente grandes como para ser detectables por difracción de rayos X. Como el parámetro de red de la fase fcc es menor que el de las muestras crudas, pero mayor que el de una dilución simple $\mathrm{Fe}-\mathrm{Cu}$, existe la posibilidad de que los carburos o átomos de carbono estén colaborando en mantener la 
distorsión en la red, a pesar de la relajación de defectos. El carburo, según los espectros Mössbauer pueden ser del tipo del carburo de Häag y el $\mathrm{Fe}_{3} \mathrm{C}$, que son magnéticos hasta $\approx 500 \mathrm{~K}$. Esta componente contribuye a que en las medidas de susceptibilidad aparezca superpuesta una línea ascendente a la respuesta de los clusters libres (el pico en la susceptibilidad).

Otro efecto del tratamiento térmico es la precipitación de $\alpha$-Fe. En los espectros Mössbauer aparece con una contribución creciente, que se observa también por DRX. También esta componente de las muestras puede ser responsable del ferromagnetismo visible en las medidas magnéticas. Este $\alpha$-Fe puede ser el resultado de la difusión promovida por el tratamiento térmico. Recordando que nuestras muestras son metaestables, que es el resultado de haber "congelado" grupos de átomos en sus posiciones, coherentes con la matriz fcc. Estos clusters, originalmente fcc en las muestras crudas, pueden haber "expulsado" al Cu de su interior, refinándose en átomos de Fe. Estos pueden haber crecido en tamaño como para hacer viable la transformación martensítica fcc $\rightarrow$ bcc, formándose así clusters de $\alpha$-Fe. De esta manera los clusters estarán constituídos por una "cáscara” fcc-Fe-Cu, y un "carozo" de $\alpha$-Fe.

El $\gamma$-Fe es otro producto del tratamiento térmico. Su contribución cae desde un $10 \%$ hasta $2,6 \%$ para $x=45 \%$ at.. En los difractogramas no es visible, bien debido a que su parámetro de red es $a=0,36467 \mathrm{~nm}$, por lo que se vería enmascarado por la aleación fcc $\mathrm{Fe}-\mathrm{Cu}$, bien porque los precipitados son pequeños y contienen pocos planos atómicos. Puede haber ocurrido también que gracias al tratamiento térmico, los átomos de hierro dispersos en la matriz fcc hayan migrado y formado pequeños cúmulos de hierro, pero no en cantidad suficiente como para perder la coherencia con la matriz fcc.

La aleación fcc $\mathrm{Fe}-\mathrm{Cu}$ cae con el aumento en la concentración. Es la parte mayoritaria de las sondas de Fe, excepto en las de $x=40$ y $45 \%$. Esta descripción es 
compatible con nuestra visión de grupos de clusters de tamaño aproximadamente constante, como se discutió en el capítulo I. Recordamos que en las muestras crudas concluíamos que el aumento en la concentración de hierro resultaba en el aumento de la cantidad de clusters ricos en Fe. Cuando hacemos el tratamiento térmico facilitamos la unión de esos clusters, en la medida en que se encuentren suficientemente próximos. Si varios clusters están próximos, al expulsar a los átomos de cobre debido a la difusión, crean una zona donde es factible la transformación $\mathrm{fcc} \rightarrow \mathrm{bcc}$, y por tanto se forma un "carozo" de $\alpha$-Fe. En las interfases tenderán a formarse los carburos con los átomos de $\mathrm{C}$ expulsados de la matriz. Es menos factible que se forme el $\gamma$-Fe en las muestras de concentración alta, debido a que hay poco espacio intercluster donde puedan estar átomos de $\mathrm{Fe}$ aislados que lo generen, o bien como la disponibilidad de interfases es mayor, esos átomos de Fe migrarán hacia sitios de $\alpha$-Fe o carburos.

Con este cuadro las interacciones magnéticas resultan complejas. El carburo y el $\alpha-\mathrm{Fe}$ son ferromagnéticos hasta más allá de la temperatura ambiente; los restos de clusters fccFe-Cu permanecerán magnéticos y los óxidos son ferrimagnéticos. Como el efecto MRG sigue siendo máximo para la concentración $x=20 \%$ y la magnetoresistividad $\Delta \rho$ de las muestras $\mathrm{Fe}-\mathrm{Cu}$ tratadas térmicamente cae, respecto a las crudas, pensamos que las razones para esto pueden ser tres:

$>$ las interacciones magnéticas múltiples “endurecen” la respuesta magnética de los clusters al campo magnético externo;

los clusters incrementan su tamaño;

el scattering dependiente del espín originado en las interfases es menor, por la menor rugosidad de los clusters. 
Esta última es coherente con lo observado en los otros trabajos, donde se veía que $\Delta \rho$ era menor luego del tratamiento térmico, y las interacciones magnéticas no eran tan complejas.

Así, podemos aseverar que la magnetoresistividad gigante en las muestras $\mathrm{Fe}-\mathrm{Cu}$ tratadas térmicamente es producida por la fase fcc-FeCu, que se encuentra mayoritariamente en las interfases de los clusters. 


\section{Capítulo III}

\section{El sistema Fe-Au}

1. Introducción

1. Trabajos previos

2. Preparación de las muestras

3. Medidas

1. Magnetoresistividad

2. Difracción de rayos $X$

3. Medidas de susceptibilidad magnética

4. Medidas de histéresis magnética

5. Espectroscopía Mössbauer

4. Tratamientos térmicos

1. Magnetoresistividad

2. Resistividad y magnetoresistividad

3. Difracción de rayos $X$

4. Susceptibilidad magnética

5. Espectroscopía Mössbauer

5. Discusión y conclusiones 


\section{Introducción}

El diagrama de fases del sistema Fe-Au (fig. 1) muestra que en la zona de concentración de hierro de alrededor del $20 \%$ at. no hay compuestos estables y es

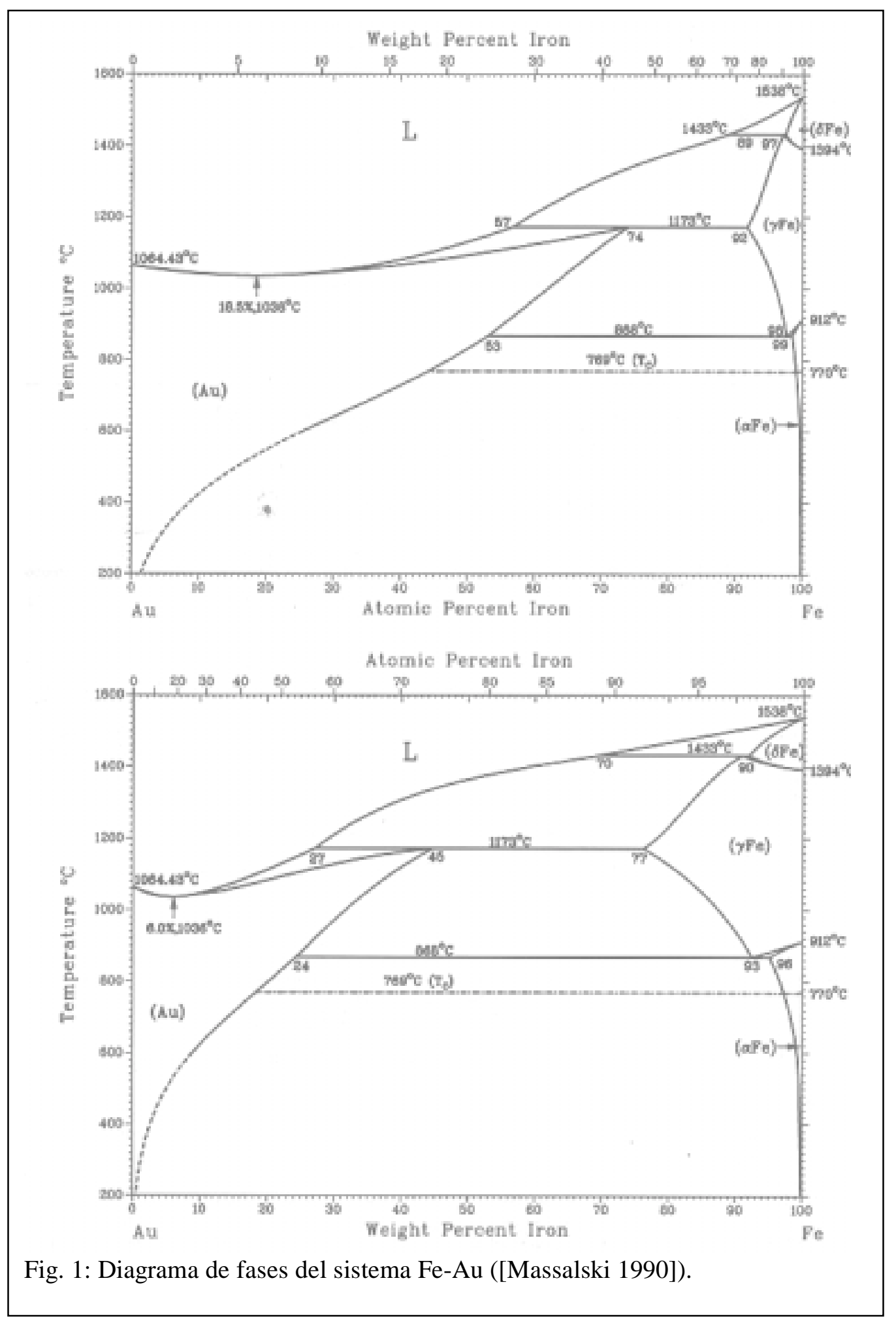


posible diluir hierro en la matriz del oro. En la zona señalada como ( $\mathrm{Au})$ la aleación terminal es fcc. El hierro queda disperso aleatoriamente en la misma, aún para concentraciones de más del $70 \%$ (alrededor de $1100{ }^{\circ} \mathrm{C}$ ). A menores temperaturas la aleación terminal es una mezcla de solución sólida $(\mathrm{Au})$ con precipitados de hierro. Existen por tanto dos comportamientos contrapuestos en las aleaciones de entre 15 y 30 \% (el rango que investigamos). El primero de ellos hará que la aleación sea homogénea, y el segundo hará que el hierro tienda a agruparse en precipitados. Esto nos hizo considerar a este sistema granular como susceptible de presentar magnetoresistividad gigante, debido a que la posibilidad de lograr una dispersión de entes magnéticos (como lo son partículas de hierro) en una matriz conductora no magnética (como la que provee el oro) es el requisito para tener dicho efecto. Como se ve del diagrama de la figura 1, la solubilidad del Fe en el Au es bastante grande a muy altas temperaturas (más de $60 \%$ ), para caer a menos de un $2 \%$ a $200^{\circ} \mathrm{C}$. Por esta razón, los métodos de preparación utilizados por los investigadores han consistido en calentar a $900{ }^{\circ} \mathrm{C}$ o más a una fundición $\mathrm{Fe}-\mathrm{Au}$ (lograda por fundido en horno de arco), para luego enfriarla violentamente y así retener la dispersión aleatoria de alta temperatura.

Como sabemos, la técnica del molido mecánico es apropiada para lograr aleaciones metaestables. En particular, es útil para lograr la dispersión de material magnético en la matriz conductora no magnética. Esto nos animó a intentar la producción de este sistema Fe-Au por aleado mecánico, lo que no había sido realizado antes. Reportamos en este capítulo los resultados obtenidos.

\subsection{Trabajos previos}

Las aleaciones Fe-Au han sido objeto de estudio desde hace por lo menos setenta años ([Shih 1931]), debido justamente al interés que presenta la posibilidad de diluir un elemento con propiedades magnéticas en una matriz no magnética. Esto generó dos 
líneas de investigación, una referida a las propiedades magnéticas en sí, y otra a las propiedades de transporte. Desde entonces, un número importante de efectos ${ }^{1}$ han sido encontrados en esta clase de aleaciones donde el hierro es minoritario. Los estudios en este sistema abarcan un rango amplio de concentraciones ${ }^{2}$, y se ha visto que existen zonas de comportamiento bien diferenciado. A muy bajas concentraciones de hierro $(<5$ \% atómico) el orden magnético está dado por la interacción RKKY, de la cual hemos hablado en §I.3??. Los átomos de hierro se encuentran solos o en configuraciones pequeñas, como dímeros o trímeros. Estas configuraciones de pequeño orden agregan a su vez interacciones dipolares o de intercambio al sistema. La suma de estas interacciones con la RKKY (mayoritaria) le dan el carácter magnético al sistema. En esta región se han estudiado en detalle el efecto Kondo y los vidrios de espín. Al aumentar la concentración de hierro, las interacciones dipolar y de intercambio van teniendo más peso, con lo que gradualmente el sistema va cambiando de carácter. Esto ocurre cuando la concentración está en el rango $5-\sim 17 \%$. Esta es la región caracterizada por el comportamiento mictomagnético o "cluster-glass", de quien hemos hablado en el capítulo I. Por encima de la concentración de percolación, como es conocida, nos encontramos con los sistemas reentrantes, o mictomagnéticos $\operatorname{percolados}^{3}$.

Rohrer publica en 1968 un trabajo muy detallado ([Rohrer 1968]) con medidas de magnetoresistividad en aleaciones $\mathrm{AuFe}$ y $\mathrm{AuMn}$ muy diluídas. Las propiedades magnetoresistivas (gigantes, aunque no se usó este nombre hasta 1988) de este sistema ya habían sido observadas en 1941 ([Nakhimovitch 1941]), para concentraciones de hasta $1 \%$ at.. Ya desde entonces se conocía que el cambio en la resistividad por

\footnotetext{
${ }_{1}^{1}$ Entre ellos, el efecto Kondo y la potencia termoeléctrica gigante.

${ }^{2}$ En [Rohrer 1968, Cannella 1975, Coles 1978] se encuentran las revisiones de estos resultados y las referencias correspondientes.

${ }^{3}$ Nos hemos guiado por la clasificación de Mydosh ([Mydosh 1993]).
} 
aplicación de un campo magnético era negativo y que había dependencia del efecto con la concentración. Posteriormente se investigó el efecto a concentraciones de hasta $10 \%$ atómico ([Nigam 1983]). Los autores no encontraron cambios sustanciales en la magnetoresistividad al pasar por la temperatura crítica indicada por el máximo de la susceptibilidad.

En 1972 se publica un trabajo ([Borg 1972]) sobre la aleación $\mathrm{Fe}_{16,6} \mathrm{Au}_{83,4}$ preparada por enfriado rápido en donde se analiza el efecto de diversos tratamientos térmicos a través de la espectroscopía Mössbauer. Los autores descubren que a muy bajas temperaturas los espectros son todos similares: un sextete ensanchado. Al aumentar la temperatura de medida el sextete colapsa a un doblete que prácticamente no cambia a temperaturas mayores. También observan que para las muestras tratadas térmicamente la transición al estado paramagnético es abrupta, a diferencia de la muestra cruda.

En [Cannella 1975] ya se propone que para concentraciones de hierro superiores al $5 \%$ atómico la contribución de clusters superparamagnéticos va siendo cada vez más importante. En la serie de trabajos de Coles y Sarkissian ([Coles 1977, Coles 1978, Sarkissian 1981]) este hecho analiza en profundidad. Estos clusters dan un carácter tipo spin-glass (llamado mictomagnético) hasta la concentración de percolación, para luego mostrarse ferromagnético (en la interpretación de estos autores). Estos últimos trabajos dieron origen a debates acerca de la reentrancia que mostraban las muestras por encima del umbral de percolación, así como del ferromagnetismo aparente en las muestras con contenidos de hierro entre 16 y $20 \%$ (ver [Beck 1985, Pureur 1999]). En el trabajo de Beck se analizan muestras en la zona de percolación. En particular, se muestra que para $x=18 \%$ no hay ferromagnetismo de largo alcance, y que las curvas de histéresis magnética se describen bien con un modelo de clusters superparamagnéticos levemente 
interactuantes. Observa además que, a la llamada "temperatura de Curie" no hay discontinuidades en el momento magnético por cluster $\mu$ ni en el coeficiente de campo molecular $\lambda$.

Ya en un trabajo anterior ([Beck 1983]) se había inferido que en una aleación relativamente concentrada en $\mathrm{Fe}(x=14,5 \%)$ un $72 \%$ de los átomos de hierro se encontraban en clusters, y que el ferromagnetismo era el orden magnético de esos clusters. También analiza que el máximo de la magnetoresistividad gigante reportada en [Hamzíc 1981] en la aleación $\mathrm{Fe}_{19} \mathrm{Au}_{81}$ era debido al máximo de la susceptibilidad.

En 1994, Wang y Xiao reportan ${ }^{4}$ la existencia de magnetoresistividad gigante en aleaciones $\mathrm{Fe}-\mathrm{Au}^{5}$ preparadas por condensación desde el estado de vapor ("magnetron co-sputtering") y calculan el camino libre medio efectivo en dichas series de muestras. Concluyen que es necesaria la separación entre una fase magnética y otra no magnética para la existencia de MRG, que este efecto es suprimido por la percolación, y que el máximo en $\Delta \rho / \rho$ ocurre entre $x=15$ y $20 \%$ en volumen de Fe.

\section{Preparación de las muestras}

Hemos producido muestras de $\mathrm{Fe}_{\mathrm{x}} \mathrm{Au}_{100-\mathrm{x}}$ en concentraciones variables, en un molino vibratorio Nissin Giken NEV-MA8 refrigerado por agua. Preparamos las concentraciones $x=15,20,25$ y $30 \%$ atómico de Fe. En todos los casos efectuamos el procedimiento que se indica a continuación: previo a cada molienda limpiamos el cilindro con arena de mar y alcohol. Luego efectuamos una molienda de un gramo de mezcla de ambos polvos en las proporciones adecuadas a la estequiometría nominal deseada. Molimos por 60 minutos y luego retiramos el material resultante. De esta manera nos aseguramos que las herramientas de molido tienen una capa de material de composición similar a lo que se va a moler, y así evitar, al menos en cierta medida, la

\footnotetext{
${ }_{5}^{4}$ [Wang 1994]

${ }^{5}$ También en los sistemas $\mathrm{Fe}-\mathrm{Ag}, \mathrm{Fe}-\mathrm{Cu}$, Co-Ag y Fe-Pt,
} 
contaminación con el acero de dichas herramientas. Posteriormente cargamos el tambor con $6 \mathrm{~g}$. en total de la mezcla de polvos. Colocamos también 43 bolas de acero de $9 \mathrm{~mm}$ de diámetro. Esto proporcionó una relación de masas de 25:1. Las purezas de los polvos de partida fueron: mayor que 99,9 \% para el $\mathrm{Au}$, y del 99,9 \% para el Fe. Las granulometrías fueron de \# 100 y 200, respectivamente. A fin de evitar la adherencia del material a las bolas y las paredes del tambor, agregamos $0,3 \mathrm{cc}$. de metanol. Cerramos los tambores en atmósfera de argón. Molimos en tiempos variables para cada una de las concentraciones, a una frecuencia nominal de $13,3 \mathrm{~Hz}$. No retiramos material del cilindro durante la molienda. Al finalizar la misma, hallamos que material resultante era polvo relativamente más fino que el oro en polvo original, y algo más claro. Los tiempos de molido fueron 20, 50 y 100 horas. 


\section{Medidas}

\subsection{Magnetoresistividad}

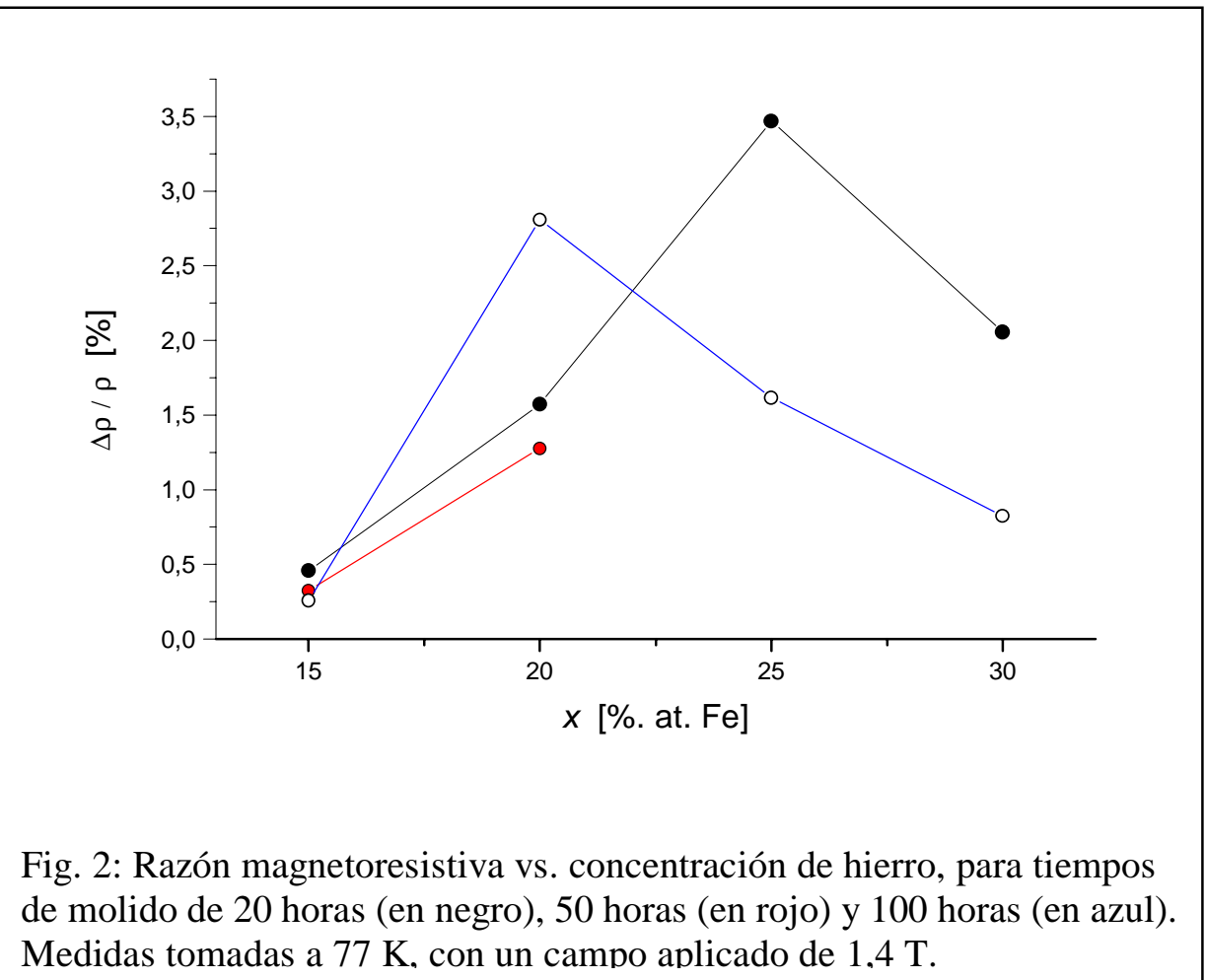

Efectuamos medidas de magnetoresistividad con el mismo arreglo experimental

que para el sistema $\mathrm{Fe}-\mathrm{Cu}$. En la figura 2 presentamos los resultados de la razón magnetoresistiva $\Delta \rho / \rho$ versus la concentración atómica de hierro $x$, para los diversos tiempos de molido.

A temperatura ambiente no fue posible detectar efecto magnetoresistivo alguno. Las características de la curva son similares a los del sistema $\mathrm{Fe}-\mathrm{Cu}$ : con el aumento de la concentración

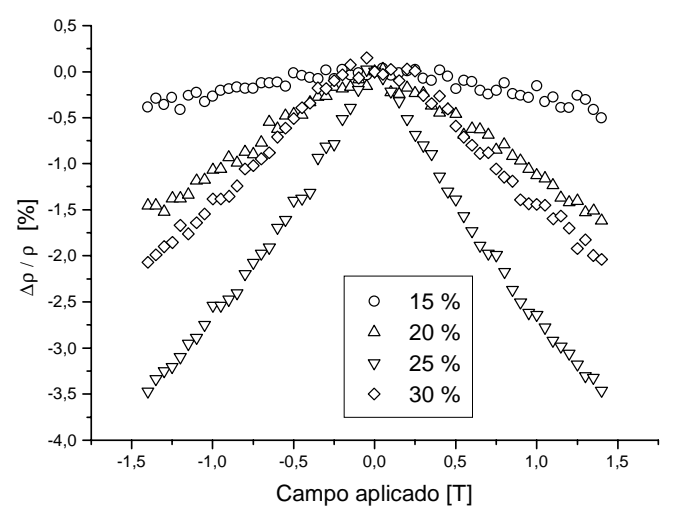

Fig. 3: Muestras con $x=15,20,25$ y $30 \%$ atómico, molidas por 20 horas y medidas a 77 K. El efecto en $\mathrm{Fe}_{5,5} \mathrm{Au}_{75}$ a 1,4 T es de 3,47\%.

crece, hasta alcanzar un máximo (en $x=25 \%$ para $\mathrm{t}=20 \mathrm{~h}$, y en $x=20 \%$ para $\mathrm{t}=100$ 
h), para luego decrecer. El máximo alcanzado fue de $\Delta \rho / \rho=3,47 \%$ para $\mathrm{t}=20$ horas.

En la figura 3 podemos ver esa medida y las de las otras concentraciones. Como en las otras muestras, no se detecta saturación ${ }^{6}$ al campo máximo aplicado, lo que puede deberse a la existencia de pequeñas partículas. También vemos solamente un máximo, por lo que el campo coercitivo ${ }^{7}$ de estas muestras es del orden o menor que el paso del campo variable con que medimos las muestras $(500 \mathrm{G})$. Tomando en cuenta que las muestras con 20 horas de molido presentan el mayor efecto magnetoresistivo, decidimos centrar nuestro análisis en ellas. Incluiremos las medidas correspondientes a los otros tiempos de molido cuando lo consideremos ilustrativo.

\subsection{Difracción de rayos $X$}

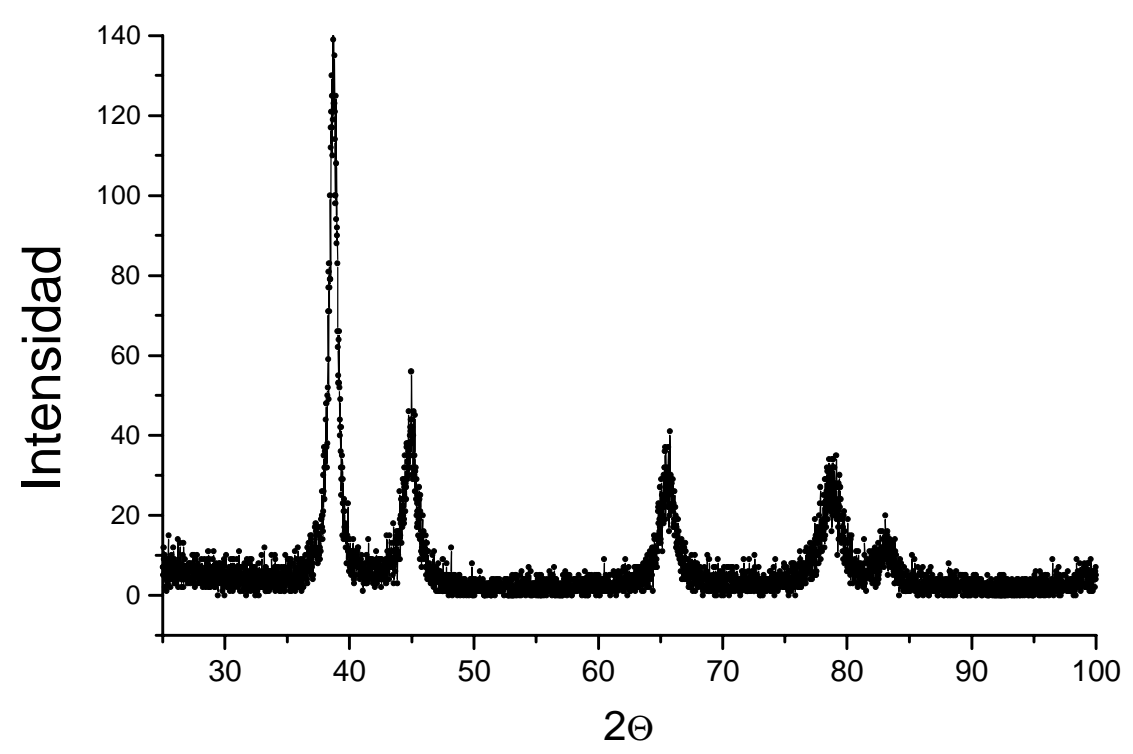

Fig. 4: Difractograma de $\mathrm{Fe}_{25} \mathrm{Au}_{75}$, después de 20 horas de molido.

Realizamos difractogramas de rayos $\mathrm{X}$ sobre todas las muestras con los

difractómetros ya mencionados en el capítulo 1. Todos los difractogramas presentan un aspecto similar al de la figura 4: los picos que aparecen son los correspondientes al Au,

\footnotetext{
${ }^{6}$ Si existiese, se visualizaría como un "pie" localizado a campos altos. Esto sería la indicación de que el sistema se orientó completamente.

${ }^{7}$ En [Xiao 1992] se muestra la correspondencia entre los máximos y el campo coercitivo. En el mismo trabajo se puede ver una muestra que presenta saturación (fig. 2 del mismo).
} 
ligeramente corridos hacia mayores ángulos, y ningún indicio de picos correspondientes al $\alpha$-Fe, a óxidos o a carburos. El Au es fcc, y tiene un parámetro de red $a=0,40782 \mathrm{~nm}$ A partir de estos difractogramas utilizamos el método descripto en el capítulo 1

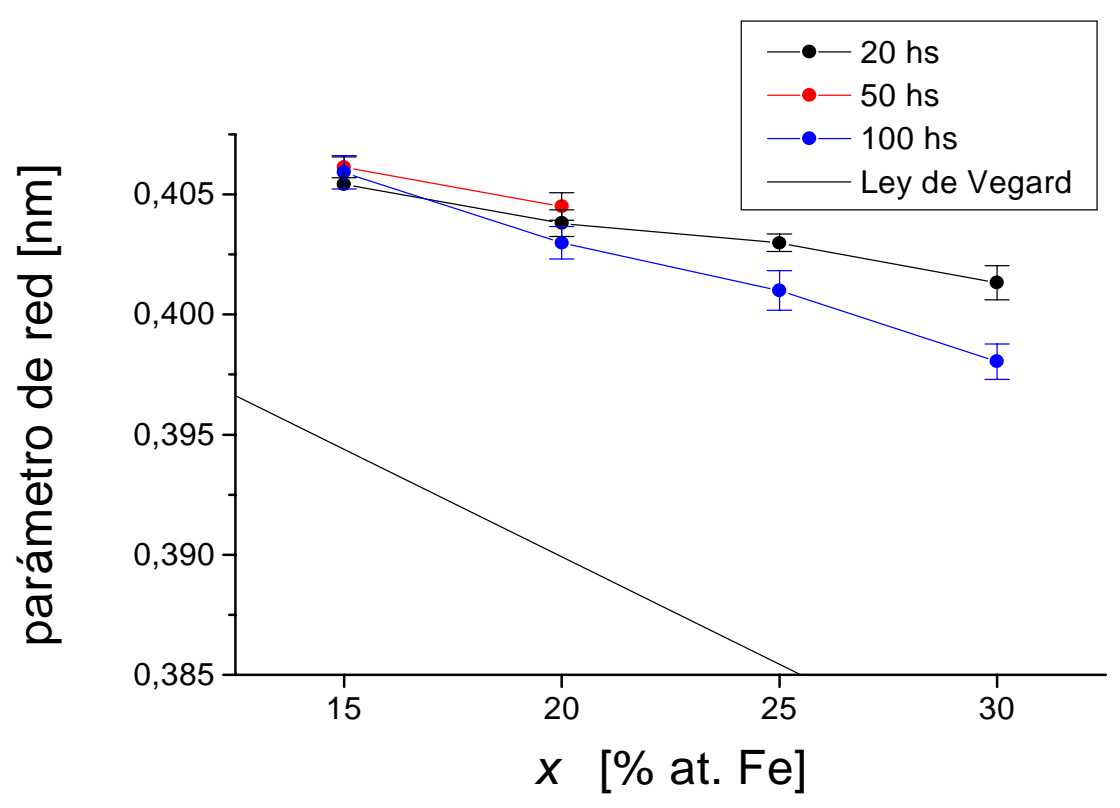

Fig. 5: Parámetro de red de las aleaciones $\mathrm{Fe}_{\mathrm{x}} \mathrm{Au}_{100-\mathrm{x}}$. La línea representa la ley de Vegard con el fcc-Fe $(a=0.36467 \mathrm{~nm})$

para obtener el parámetro de red. A diferencia del sistema Fe-Cu no hubo que descartar reflexiones. En la figura 5 vemos el parámetro de red calculado a partir de nuestras medidas, en función de la concentración de hierro. Los parámetros de red de todas las muestras caen por encima de lo previsto por la ley de Vegard, si tomamos como extremo derecho al $\gamma$-Fe. Todas ellas tienen un parámetro de red menor al del Au puro. El parámetro decrece con la concentración de Fe, lo que es plausible debido a que el volumen atómico del Fe es menor que el del Au. Así, al haber

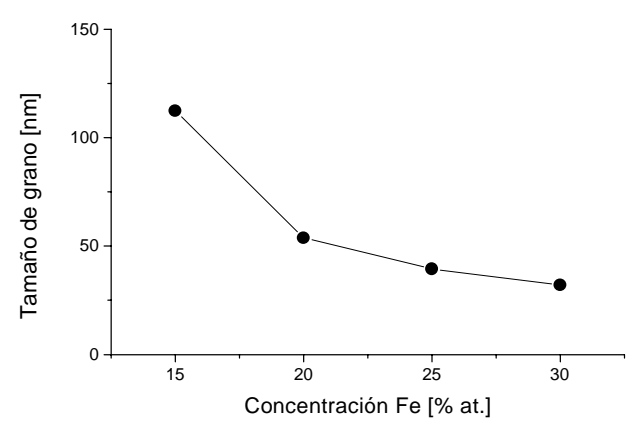

Fig. 6: Tamaño final de grano calculado de acuerdo al método del ancho integrado rK1n 10717 
más átomos de Fe disponibles, más zonas en la red quedan "acortadas" debido a átomos de Fe sustitucionales. El decrecimiento del parámetro de red es monótono con $x$, lo que es contrario a pensar que el efecto magnetoresistivo es causado por un cambio en el parámetro de red que afecte a la integral de intercambio, como se discutió en §I.4.

De la misma manera que en el sistema $\mathrm{Fe}-\mathrm{Cu}$ hemos calculado el tamaño de grano. En la figura 6 es apreciable, a pesar del que el error es del orden de los valores medidos, que el tamaño de grano disminuye con la concentración de hierro. Los tamaños de las muestras con 20 horas de molido caen desde unos $112 \mathrm{~nm}$ para $x=15 \%$ hasta aproximadamente $32 \mathrm{~nm}$ para $x=30 \%$. Estos valores son superiores a los del sistema $\mathrm{Fe}-\mathrm{Cu}$, aunque el comportamiento con la concentración es el mismo. 


\subsection{Susceptibilidad magnética}

Efectuamos medidas de susceptibilidad dinámica ${ }^{8}$ utilizando la técnica de enfriado sin campo (ZFC), y a cada temperatura medimos la susceptibilidad a distintas frecuencias. Las frecuencias utilizadas abarcaron las cuatro décadas accesibles con el equipo $(\omega \leq 10 \mathrm{kHz})$. En general medimos con un campo de $1 \mathrm{Oe}$, a fin de evitar fenómenos de "planchado" de los picos, como fue observado en otros trabajos ([Sarkissian 1981]). En la figura 7 mostramos los resultados. Para $x=15$ y $20 \%$ los comportamientos son similares: La susceptibilidad crece al aumentar la temperatura

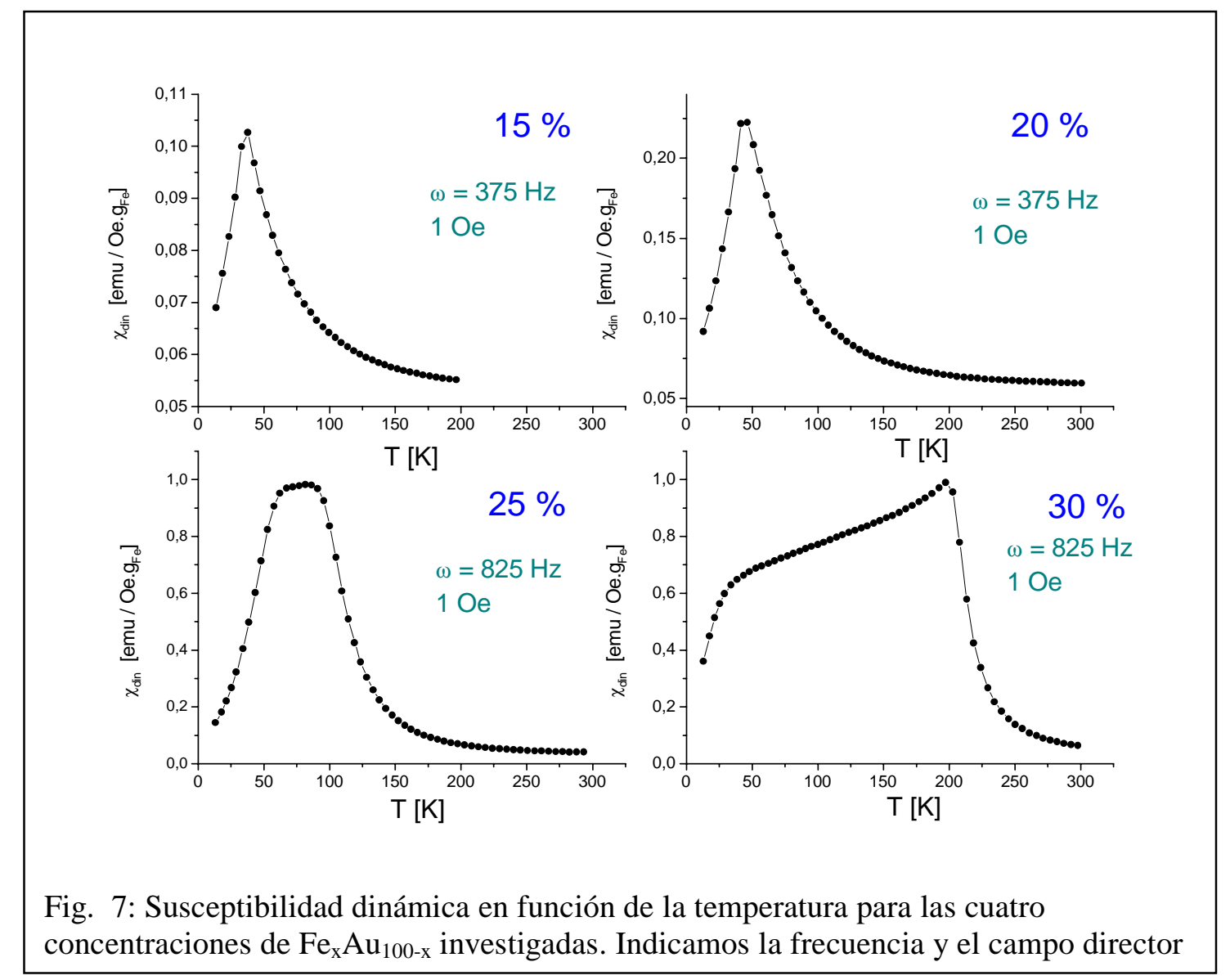

hasta alcanzar un máximo. Luego decrece monótonamente. La forma de ambas curvas

es la de un sistema mictomagnético o "cluster-glass", tal como lo observado por

Sarkissian y Coles en este mismo sistema. Para la muestra con $25 \%$ atómico de Fe,

\footnotetext{
${ }^{8}$ Con el equipo LakeShore mencionado en el apéndice C
} 
aunque la zona de bajas temperaturas es similar a las anteriores, la zona de máximo ya no es un pico agudo, sino redondeado. Descartamos que la causa de esto sea el efecto desmagnetizante, como fue puntualizado por Beck. En su trabajo ([Beck 1985]) observa que la susceptibilidad medida $\chi_{\mathrm{e}}$ está afectada por el factor desmagnetizante $D$, ya que al campo externo $H_{e}$ debe serle descontado ese efecto para tener el campo interno:

$$
H_{i}=H_{e}-D \sigma,
$$

con $\sigma$ la magnetización por unidad de masa. Como la susceptibilidad medida es

$\chi_{\mathrm{e}}=\sigma / H_{e}$, se ve entonces que

$$
\chi_{e}=\frac{1}{\left(\chi^{-1}+D\right)}
$$

Por lo tanto, cuando la susceptibilidad interna es muy grande, su recíproca es pequeña, y puede serlo también respecto al factor desmagnetizante: $\chi_{i}^{-1}<<$. De esta manera, hay una zona donde la susceptibilidad medida es insensible a la temperatura, ya que entonces

$\chi_{\mathrm{e}} \approx 1 / \mathrm{D}$

Esto no ocurre, ya que para la muestra $\mathrm{Fe}_{30} \mathrm{Au}_{70}$ es posible medir un pico nítido en la susceptibilidad con un valor de $\chi$ similar $\left(0,99 \mathrm{emu} / \mathrm{G} . \mathrm{g}_{\mathrm{Fe}}\right.$, contra $\operatorname{los} \approx 0,98 \mathrm{emu} / \mathrm{G} . \mathrm{g}_{\mathrm{Fe}}$ de $\left.\mathrm{Fe}_{25} \mathrm{Au}_{75}\right)$.

La muestra con $x=30 \%$ está ya percolada magnéticamente, como es visualizable por la existencia de un "hombro" (la reentrancia) a $\mathrm{T} \approx 25 \mathrm{~K}$ y un pico aguzado a $\mathrm{T} \approx 200 \mathrm{~K}$.

El comportamiento de las muestras $\mathrm{Fe}_{15} \mathrm{Au}_{85}$ y $\mathrm{Fe}_{30} \mathrm{Au}_{70}$ es similar al descripto por Sarkissian para las muestras por debajo y por encima del umbral de percolación. Las muestras de $\mathrm{Fe}_{20} \mathrm{Au}_{80}$ y $\mathrm{Fe}_{25} \mathrm{Au}_{75}$ se muestran diferentes de lo observado en el trabajo mencionado para esas mismas concentraciones. La primera no muestra signos de haber 
percolado, es decir que se trataría de un sistema mictomagnético por debajo del umbral de percolación. La segunda pareciera todavía pertenecer al primer grupo, ya que el comportamiento a $\mathrm{T}<50 \mathrm{~K}$ es similar (la convexidad de la curva es la misma que en las anteriores), pero el pico redondeado muestra que es posible o bien la existencia de dos tamaños de cluster suficientemente diferenciados o

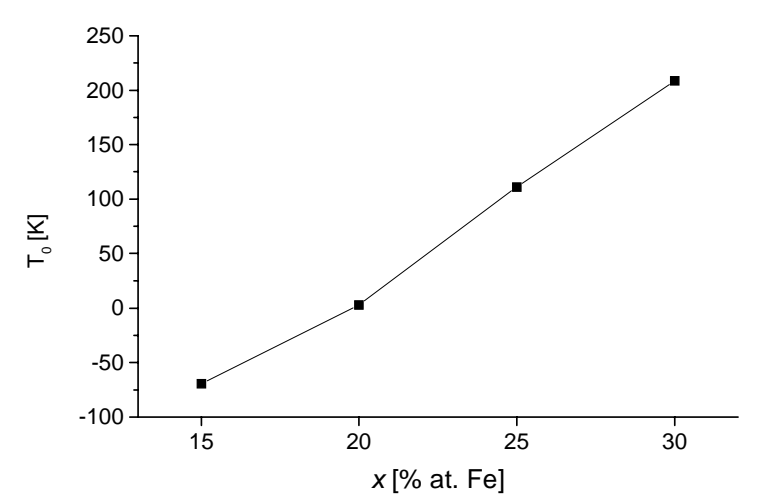

Fig. 8: Temperatura de Weiss vs. concentración de

bien otro efecto que no hemos podido dilucidar. La muestra de $\mathrm{Fe}_{25} \mathrm{Au}_{75}$ parece ser un estado intermedio entre las muestras con $x=20$ y 30 .

Calculamos la temperatura de Weiss $\theta$ a través de la inversa de la susceptibilidad. Como la ley de Curie-Weiss es

$$
\chi=\frac{C t e}{T-\theta}
$$

ajustando la zona de altas temperaturas a una recta, la intecepción de $1 / \chi$ con el eje $\mathrm{T}$ nos da el valor de $\theta$. En la figura 8 mostramos el resultado para todas las muestras. Para $x=15 \%$ la temperatura de Weiss es negativa, lo que indica que el alineamiento intercluster es antiferromagnético. Para las otras concentraciones es positivo, lo que indica alineamiento ferromagnético. La temperatura crece con la concentración, lo que nos dice que la interacción de intercambio es cada vez más grande.

\subsection{Histéresis magnética}

Hemos realizado curvas de histéresis a temperatura ambiente en un magnetómetro de muestra vibrante comercial. Las muestras analizadas no muestran 
saturación (figura 9). En el caso de $\mathrm{Fe}_{25} \mathrm{Au}_{75}$ ni siquiera a $8 \mathrm{~T}$. Esto es indicativo de la existencia de pequeñas partículas magnéticas. El campo coercitivo para $x=15$ y $20 \%$ es bastante similar: $H_{c} \approx 580 \mathrm{G}$. No fue posible hacer un ajuste de la magnetización con una función de Langevin.

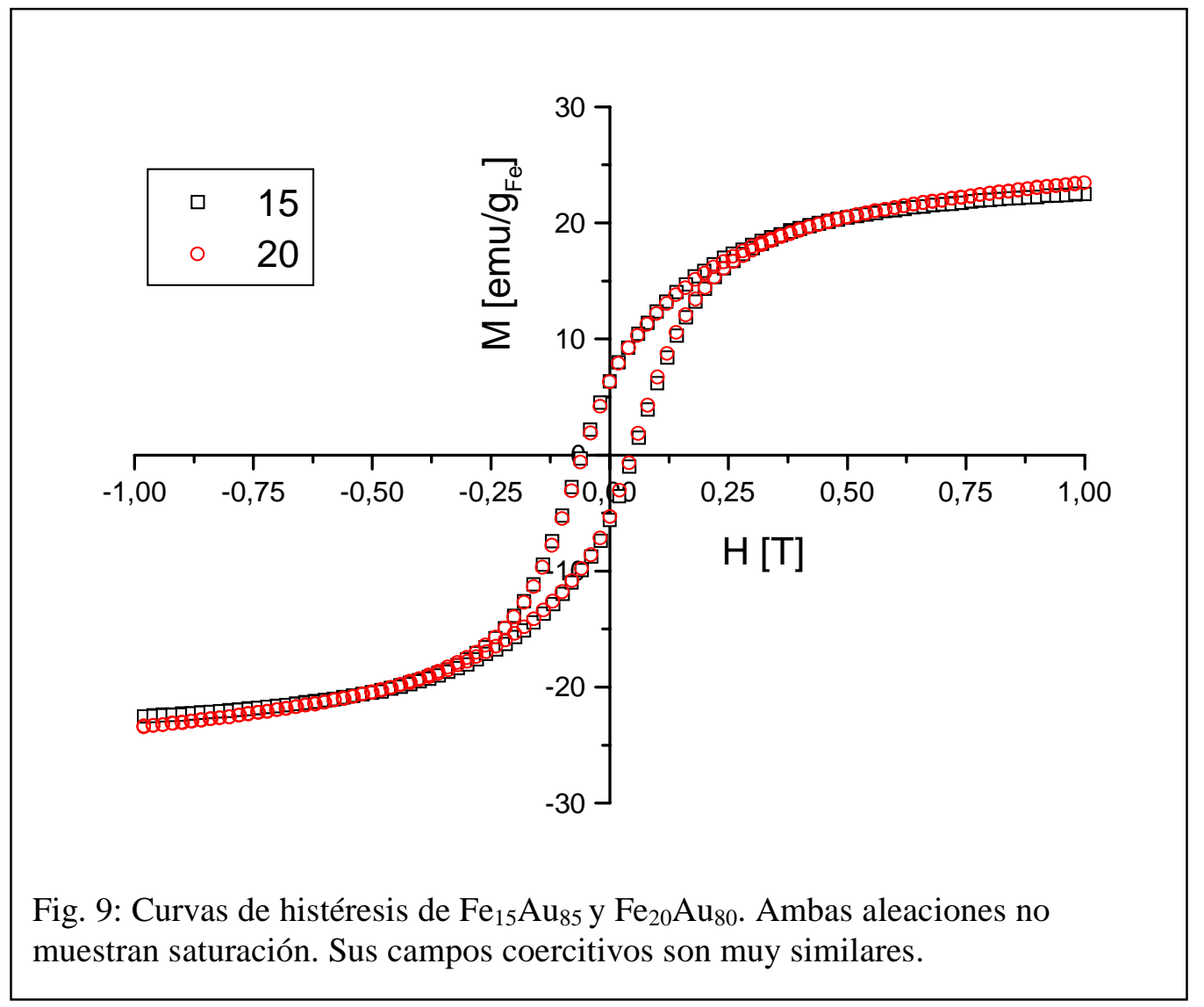




\subsection{Espectroscopía Mössbauer}

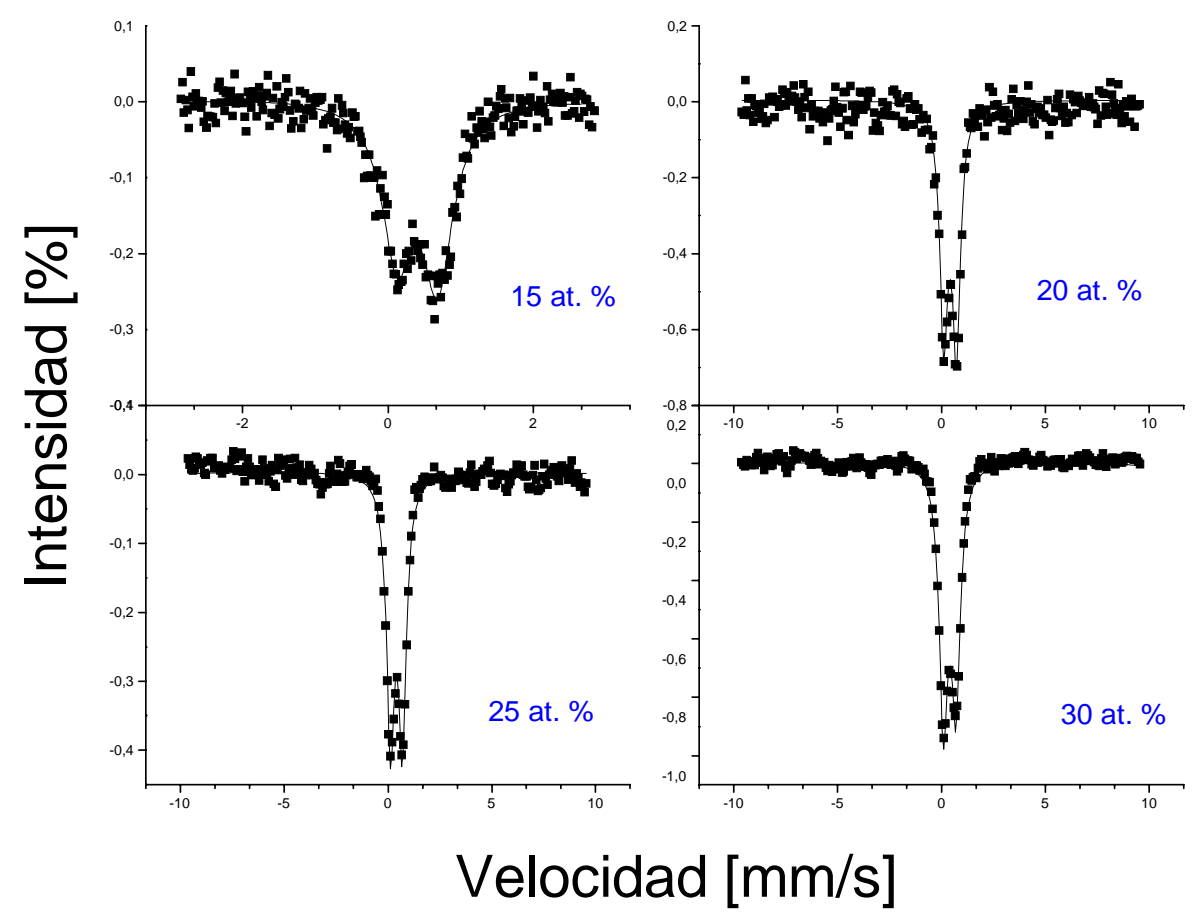

Fig. 10: Medidas Mössbauer tomadas a temperatura ambiente. El espectro para $x=$ $15 \%$ está tomado en un rango de velocidades menor a las otras. Las líneas corresnonden al aiuste.

Realizamos espectroscopía Mössbauer sobre estas muestras. La obtención de los espectros ha sido dificultosa debido a la gran absorción de la radiación gamma en el Au. Como no utilizamos hierro enriquecido en el isótopo ${ }^{57} \mathrm{Fe}$, y las muestras eran polvos gruesos, no fue posible tener un buen espectro para $x=15 \%$. En la figura 10 presentamos resultados para las muestras con 20 horas de molido. Los espectros no difieren sustancialmente entre sí. Como vemos, todos muestran un doblete, levemente asimétrico como el ya visto en las aleaciones $\mathrm{Fe}-\mathrm{Cu}$, pero de parámetros hiperfinos distintos a los del sistema anterior (ver Tabla I). Los corrimientos isoméricos son más elevados que en aquellas aleaciones $\mathrm{Fe}-\mathrm{Cu}$, pero menores que lo reportado en la literatura para el sistema $\mathrm{Fe}-\mathrm{Au}$ preparado por otras vías $(\delta=0,628 \mathrm{~mm} / \mathrm{s}$, [Shenoy 1978]). El ancho de línea $\Gamma$ es grande: varía entre 0,45 y $0,51 \mathrm{~mm} / \mathrm{s}$. Como este 
parámetro está relacionado con la existencia de diferentes sitios, este ensanchamiento puede ser debido a la existencia de una dispersión de tamaños. La asimetría entre las líneas 1 y 2 aumenta ligeramente con la concentración, desde 0,86 a 1,09. No hay indicios de $\alpha$-Fe (ni aún SPM), o de $\gamma$-Fe.

\begin{tabular}{|c|c|c|c|c|}
\hline \hline Concentración & $\Delta[\mathrm{mm} / \mathrm{s}]$ & $\delta[\mathrm{mm} / \mathrm{s}]$ & $\begin{array}{c}\text { Asimetría } \\
\left(\mathrm{L}_{1} / \mathrm{L}_{2}\right)\end{array}$ & $\Gamma[\mathrm{mm} / \mathrm{s}]$ \\
\hline 15 & $0,56_{0,01}$ & $0,51_{0,01}$ & $0,86_{0,04}$ & $0,51_{0,02}$ \\
\hline 20 & $0,59_{0,01}$ & $0,52_{0,01}$ & $0,96_{0,04}$ & $0,46_{0,02}$ \\
\hline 25 & $0,57_{0,01}$ & $0,51_{0,01}$ & $1,01_{0,03}$ & $0,45_{0,01}$ \\
\hline 30 & $0,58_{0,01}$ & $0,50_{0,01}$ & $1,09_{0,02}$ & $0,49_{0,01}$ \\
\hline
\end{tabular}

Tabla I: Parámetros hiperfinos medidos en las muestras con 20 horas de molido. 


\section{Tratamientos térmicos}

Buscamos un tratamiento térmico que mejorase el efecto magnetoresistivo $\Delta \rho / \rho$.

Para ello tomamos las muestras compactadas y las cerramos en ampollas de vidrio o cuarzo en una atmósfera mezcla de argón e hidrógeno (en una relación 2:1), a baja presión (0,3 atm). Colocamos las ampollas en hornos eléctricos con control de rampa. Probamos diversos regímenes de tratamiento térmico, variando la temperatura y el tiempo de tratamiento térmico. Encontramos que la temperatura óptima es de alrededor de $500{ }^{\circ} \mathrm{C}$ (en la figura 11

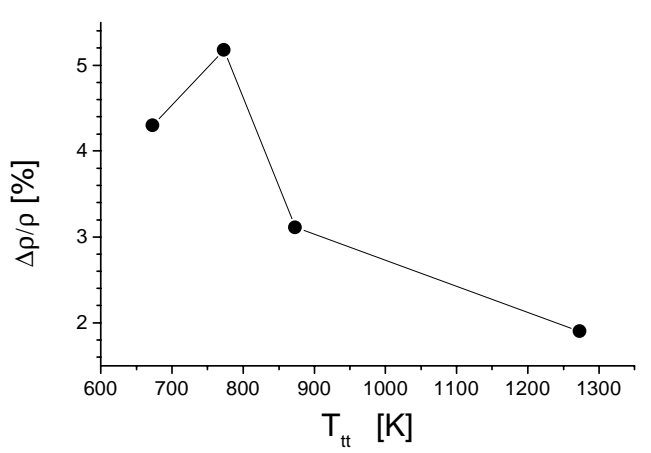

Fig. 11: Efecto magnetoresistivo a 1,4 T y $77 \mathrm{~K}$ para la muestra $\mathrm{Fe}_{25} \mathrm{Au}_{75}$ con 20 horas de molienda, en función de la temperatura

presentamos el efecto magnetoresistivo en función de la temperatura del tratamiento térmico). Para esta temperatura, el tiempo óptimo de tratamiento es de 24 horas.

\subsection{Magnetoresistividad}

Las medidas de magnetoresistividad mostraron el mismo comportamiento con la concentración que las
correspondientes
muestras "crudas"
como se ve en la figura
12 , aunque con un
efecto mayor (como se
ve en el inserto de la
misma). La muestra de $_{F_{25} \mathrm{Au}}$ molida por 20

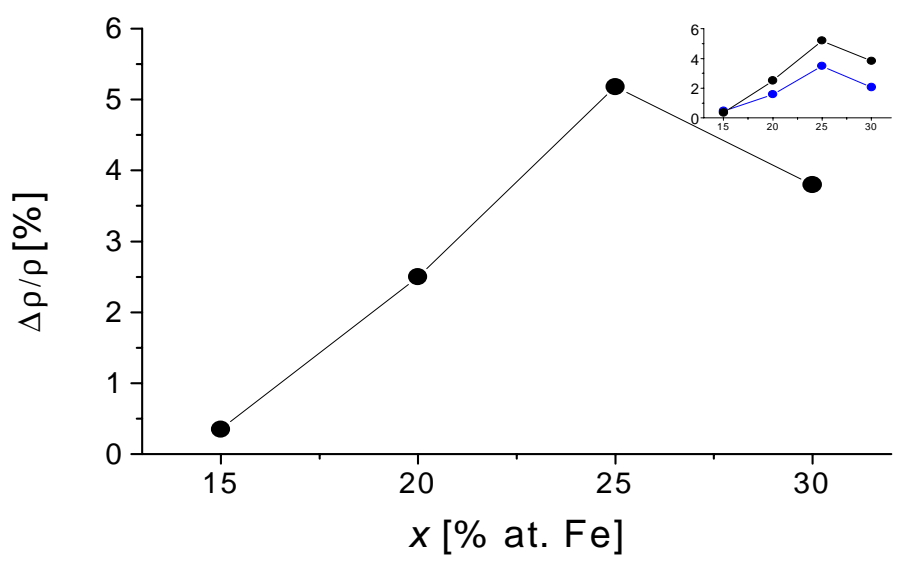

Fig. 12: Efecto magnetoresistivo en función de la concentración de Fe, medido a $77 \mathrm{~K}$. En el inserto: la

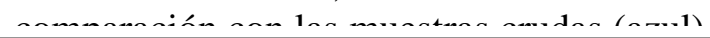

horas y tratada térmicamente mostró el mayor efecto, con $\Delta \rho / \rho=5,18 \%$ a $77 \mathrm{~K}$. Esa 
medida se muestra en la

figura 13. Es similar a las de otras concentraciones en aspecto, así como a las de las muestras crudas. No aparecen aquí tampoco dos máximos, lo que indica que la coercitividad permanece baja con el tratamiento

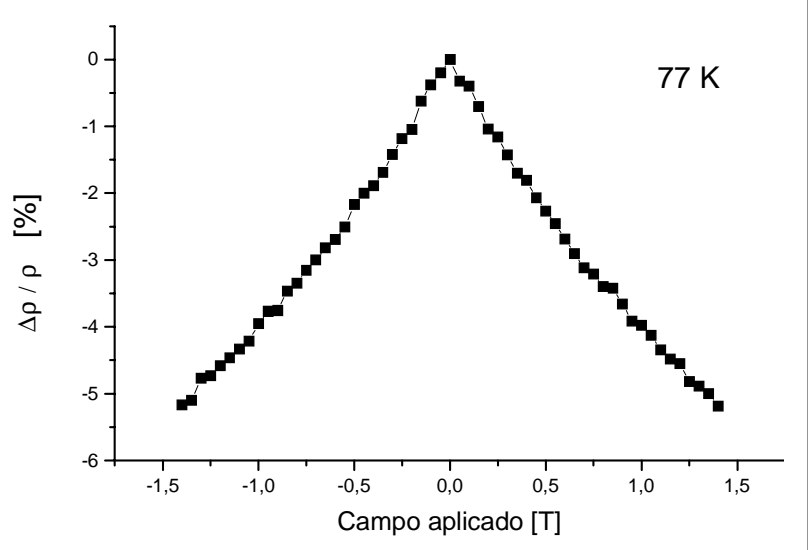

Fig. 13: Magnetoresistividad de la muestra de $\mathrm{Fe}_{25} \mathrm{Au}_{75}$ tratada térmicamente.

térmico. A diferencia de las del sistema $\mathrm{Fe}-\mathrm{Cu}$ tratadas térmicamente, la medida de la magnetoresistividad no presenta dispersión en los puntos.

\subsection{Resistividad y magnetoresistividad}

En la figura 14 presentamos las medidas de resistividad pura $\rho$ y la diferencia $\Delta \rho$ para las muestras crudas y tratadas térmicamente (TT). Como vemos, la resistividad pura es menor en las muestras tratadas térmicamente. Para $x=15$ y $20 \%$ es

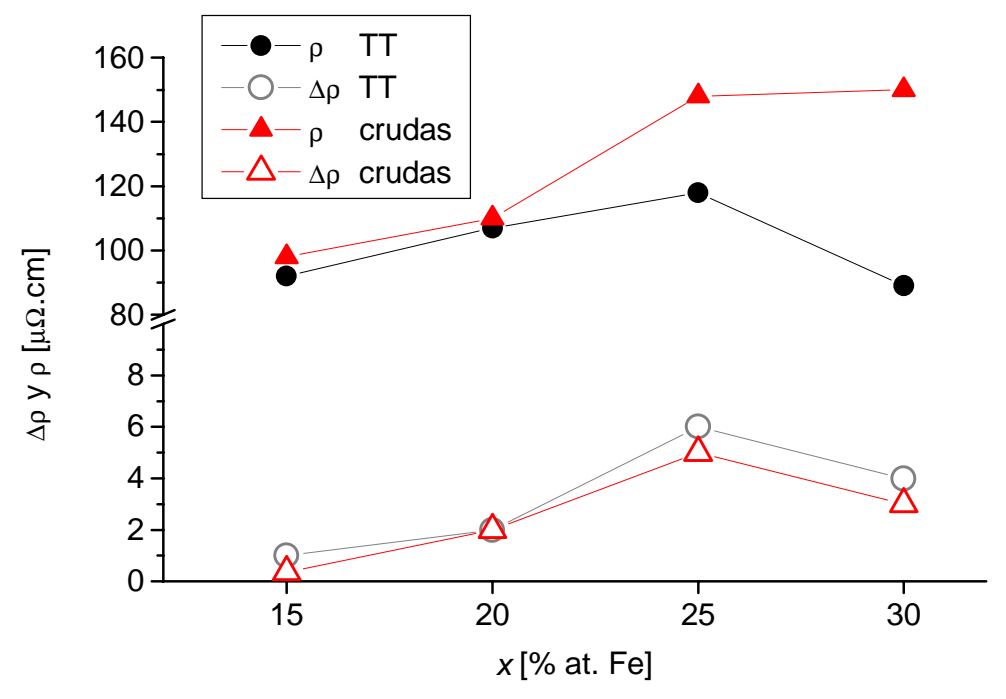

Fig. 14: $\Delta \rho$ y $\rho$ de las muestras crudas (en rojo) y tratadas térmicamente (en negro) medidas a $77 \mathrm{~K} . \Delta \rho$ fue tomada con un 
prácticamente la misma que en las correspondientes crudas, luego la diferencia se ensancha hasta ser un $60 \%$ menor (para $\mathrm{Fe}_{30} \mathrm{Au}_{70}$ tratada térmicamente). La resistividad pura en ambos tipos de muestras se encuentra dentro de los valores esperables para una mezcla con impurezas y/o amorfa. La diferencia en resistividad cuando aplicamos un campo magnético está dada por $\Delta \rho$ (lo que dice que esta magnitud está relacionada con la dispersión de origen magnético). Como vemos, no hay mayor diferencia en ambos tipos de muestra. El máximo se da en $x=25 \%$ en ambos casos, con un valor de 5 y 6 $\mu \Omega \mathrm{cm}$ para la muestra cruda y tratada térmicamente, respectivamente. Estos valores son mayores que los obtenidos por coevaporación por el grupo de Xiao. Ellos miden $\Delta \rho=$ $3,97 \%$ (a 4,2 K y 1,4 T). Esto puede deberse bien a que los clusters producidos por el aleamiento mecánico alcanzan una combinación de tamaño y distancia a sus vecinos más efectiva, o bien que las interfases de nuestros clusters son más rugosas que en los producidos por coevaporación.

\subsection{Difracción de rayos $X$}

Las medidas de difracción de rayos $\mathrm{X}$ las realizamos con un aparato Philips

PW1170. Los difractogramas resultantes no son muy diferentes a los de las muestras crudas. Los picos de la zona explorada $\left(20^{\circ} \leq\right.$ $\left.2 \theta \leq 100^{\circ}\right)$ son los correspondientes al $\mathrm{Au}$, ligeramente corridos hacia ángulos mayores. En base al

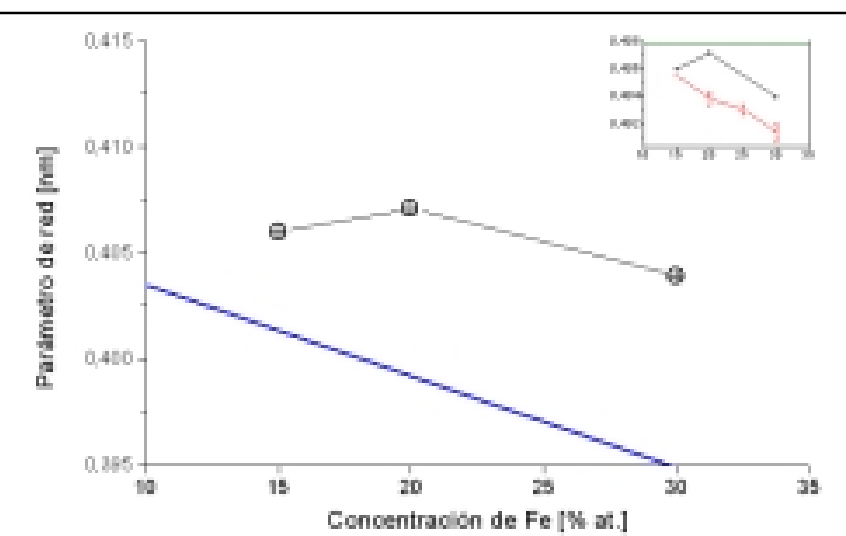

Fig. 15: Parámetro de red de las muestras $\mathrm{Fe}_{\mathrm{x}} \mathrm{Au}_{100-\mathrm{x}}$ tratadas térmicamente en función de la concentración. La línea azul es la ley de Vegard. En el inserto vemos la comparación con las muestras crudas. La línea verde oscuro de su extremo

método explicado en §I.3.2, hemos calculado el parámetro de red. Como vemos en la 
figura 15, el parámetro de red en estas muestras crece hasta 0,4071 $\pm 0,0001 \mathrm{~nm}$ en $x=$ $20 \%$ y luego decrece para $x=30 \%$. Sistemáticamente estos parámetros son mayores que los de las muestras crudas (ver el inserto de la misma figura), y por tanto también superiores a lo que indica la Ley de Vegard. Como también se ve en ese inserto, ninguna de las muestras alcanza a tener el parámetro de red original del oro, aunque en el caso de las muestras tratadas es mucho más próximo.

Los picos (200) y (222) del oro, ubicados a $44,4^{\circ}$ y $81,7^{\circ}$ respectivamente, presentan una deformación. No es posible ajustar con un solo par de lorentzianas como corresponde a una reflexión vista con los rayos $\mathrm{K}_{\alpha 1}$ y $\mathrm{K}_{\alpha 2}$. Para hacerlo correctamente hace falta otro par, como si estuviera presente una reflexión adicional. Asimismo las alturas relativas de estos picos y el (220) al pico de mayor intensidad (el (111)) no son

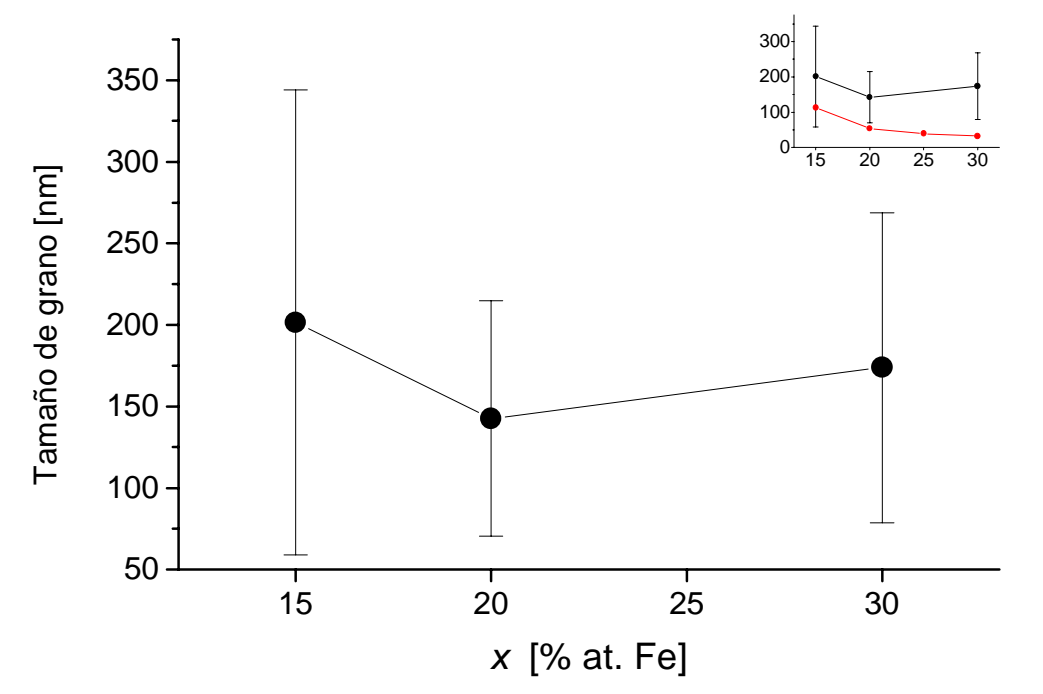

Fig. 16: Tamaño de grano vs. concentración en las muestras tratadas térmicamente. En el inserto: Comparación con las muestras crudas

las esperables, de acuerdo a los valores aceptados para el oro en bloque ${ }^{9}$. Como en $44,7^{\circ}$

, $65,08^{\circ}$ y $82,4^{\circ}$ hay reflexiones del $\alpha-\mathrm{Fe}^{10}$ es posible que las anomalías se deban a la 
precipitación de éste. Los difractogramas no tienen la suficiente estadística para intentar un ajuste confiable con dos pares de lorentzianas. Por lo tanto, sólo podemos sospechar de la existencia de $\alpha$-Fe precipitado.

Calculamos el tamaño de grano con todos los picos visibles correspondientes al Au en cada difractograma, siguiendo el método de Klug y Alexander anteriormente descripto en §I.3.2 (ver figura 16). Éste es consistentemente más grande en las muestras tratadas térmicamente que en las muestras crudas (ver inserto de la misma figura). Parece mantenerse constante con la concentración con valores promedio entre 143 y 202 $\mathrm{nm}$. 


\subsection{Medidas de susceptibilidad magnética}

Las medidas de susceptibilidad las efectuamos con el equipo LakeShore

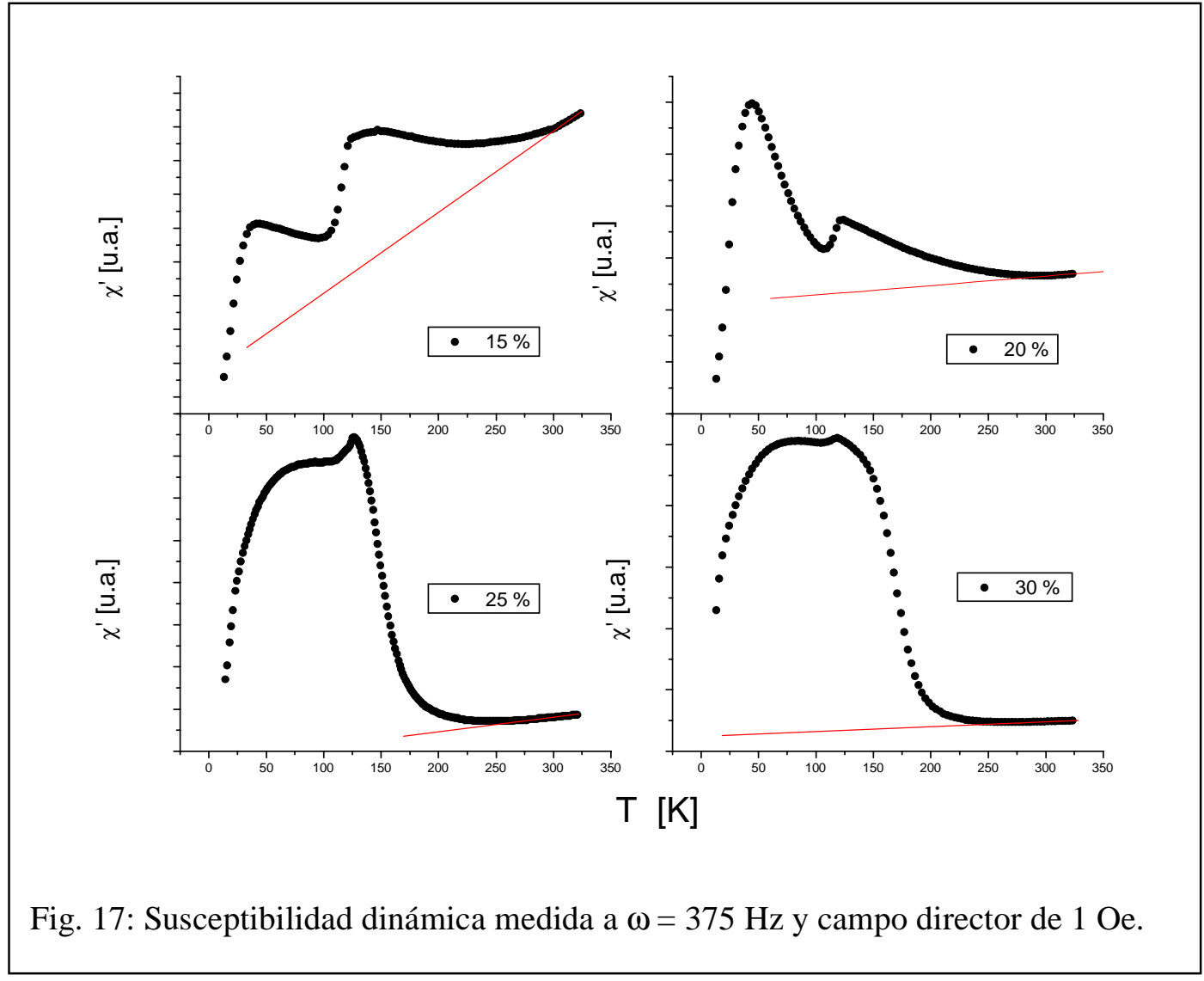

aplicando diversas frecuencias y un mismo campo director de 1 Oe. Las medidas (figura 17) presentan efectos superpuestos: para las muestras con $x=15$ y $20 \%$ podemos ver dos máximos, localizados a aproximadamente 40 - $50 \mathrm{~K}$ y a 123 - $136 \mathrm{~K}$. Por el comportamiento a muy baja temperatura (la zona cóncava hacia arriba, ubicada entre 13

y $25 \mathrm{~K})$ podemos pensar que estas dos muestras tienen un comportamiento tipo mictomagnético no percolado. Ambos aparecen "montados" sobre una curva ascendente (líneas rojas de la figura 17), que puede provenir de una componente ferromagnética. Esto también está ocurriendo en las muestras más concentradas $(x=25$ y $30 \%)$. A pesar que el aspecto de ambas es el de un mictomagneto percolado, en ambas es distinguible la recta ascendente. Existe una anomalía en la zona de reentrancia de estas dos medidas. 
Se visualiza como un cambio en la pendiente a $\mathrm{T} \approx 23 \mathrm{~K}$. El máximo en la susceptibilidad está ubicado a $\mathrm{T}=119-126 \mathrm{~K}$ en estas dos últimas muestras, en coincidencia con los de las muestras anteriores. La susceptibilidad aumenta con la concentración.

\subsection{Espectroscopía Mössbauer}

Intentamos obtener espectros Mössbauer de las muestras tratadas térmicamente. El tratamiento térmico sinterizó los lingotes sobre los cuales se hicieron las medidas aquí reportadas, con lo que ya no fue posible pulverizarlos para preparar el absorbente, como habíamos hecho en el sistema $\mathrm{Fe}-\mathrm{Cu}$. Tampoco fue posible tratar térmicamente los polvos originales obtenidos de la molienda y obtener los mismos difractogramas que antes. El método de tratamiento que intentamos (vacío dinámico en lugar de atmósfera Ar $+\mathrm{H}$ ), además de ampollas de cuarzo de distinta calidad que las originales, dio lugar a precipitaciones diferentes a las ya obtenidas con los lingotes, como comprobamos por medio de la DRX.

\section{Discusión y conclusiones}

El trabajo mecánico, por medio del "bombeo de soluto por dislocaciones" anteriormente descripto, promueve la dispersión coherente del Fe en la matriz del Au. Esto produce una reducción del parámetro de red. Éste es inferior al del Au puro, pero más grande que si se tratase de una dilución simple, como lo indica el hecho de que los valores predichos por la ley de Vegard son inferiores a nuestros resultados. La difracción de rayos X y la espectroscopía Mössbauer confirman que no queda hierro bcc luego de 20 horas de molido. Todas las muestras presentan MRG, lo que indica que hemos logrado la dispersión de una fase magnética en la matriz del Au. El calor positivo de mezcla (8 kJ/mol, según [De Boer 1989]) puede ser el responsable de la clusterización del Fe en el Au, así como la obtención de distancias interatómicas por 
encima de lo predicho por la ley de Vegard. El efecto MRG tiene un comportamiento variable con la concentración. Aumenta desde $x=15 \%$ para alcanzar su máximo valor en $x=25 \%$ (para 20 horas de molido), y luego decrecer en $x=30 \%$. Estas medidas no muestran saturación, como corresponde a un sistema de partículas pequeñas. Ni siquiera se ve un comportamiento histerético en la medida de magnetoresistividad de $\mathrm{Fe}_{30} \mathrm{Au}_{70}$, lo que da la pauta de la inexistencia de un cluster de tamaño macroscópico. Las medidas magnéticas muestran un sistema mictomagnético, que percola para alguna concentración por encima de $x=25 \%$. Los tamaños de granos promedio de todas las muestras caen por debajo de los $150 \mathrm{~nm}$ (según los difractogramas), lo que hace que los clusters, ricos en átomos de hierro, sean menores que esa longitud y por tanto susceptibles de ser superparamagnetos.

A fin de discernir entre si se trata de un superparamagneto o un vidrio de espín, calculamos el número $\mathrm{W}$ :

$$
W=\Delta T_{c} /\left[T_{c} \Delta \log (\omega)\right] \quad,
$$

donde $\mathrm{T}_{\mathrm{c}}$ es la temperatura crítica (del máximo en $x=15$ y $20 \%$, y del punto de inflexión en $x=25$ y $30 \%), \Delta \mathrm{T}_{\mathrm{c}}$ es el corrimiento en temperatura para dos frecuencias diferentes, y $\omega$ la frecuencia del campo aplicado. Para todas las temperaturas y muestras los resultados fueron inferiores a 0,009, como ocurre en los vidrios de espín canónicos [Mydosh 1993, Goya 2001].

Como comentamos en §III.1.1, las aleaciones muy diluídas Fe $\underline{\mathrm{Au}}$ son uno de los vidrios de espín canónicos. La interacción magnética dominante es la RKKY. Al aumentar la concentración de la fase magnética, aparecen interacciones entre racimos como la dipolar o la de intercambio. Al pasar el umbral de percolación, muchos clusters se encuentran en contacto directo o muy próximos, con lo que la interacción dominante en el sistema ya no es la RKKY sino alguna de las dos antes mencionadas, que va 
haciendo que la interacción total entre clusters aumente, como se refleja en la figura 8.

De todas maneras, aún a $x=30 \%$ atómico todavía existen clusters que pueden alinear sus espines con el campo aplicado, dando todavía efecto magnetoresistivo gigante.

Implicancias para el diagrama de fases magnéticas:

Calculamos las temperaturas críticas de acuerdo a lo discutido en el subcapítulo

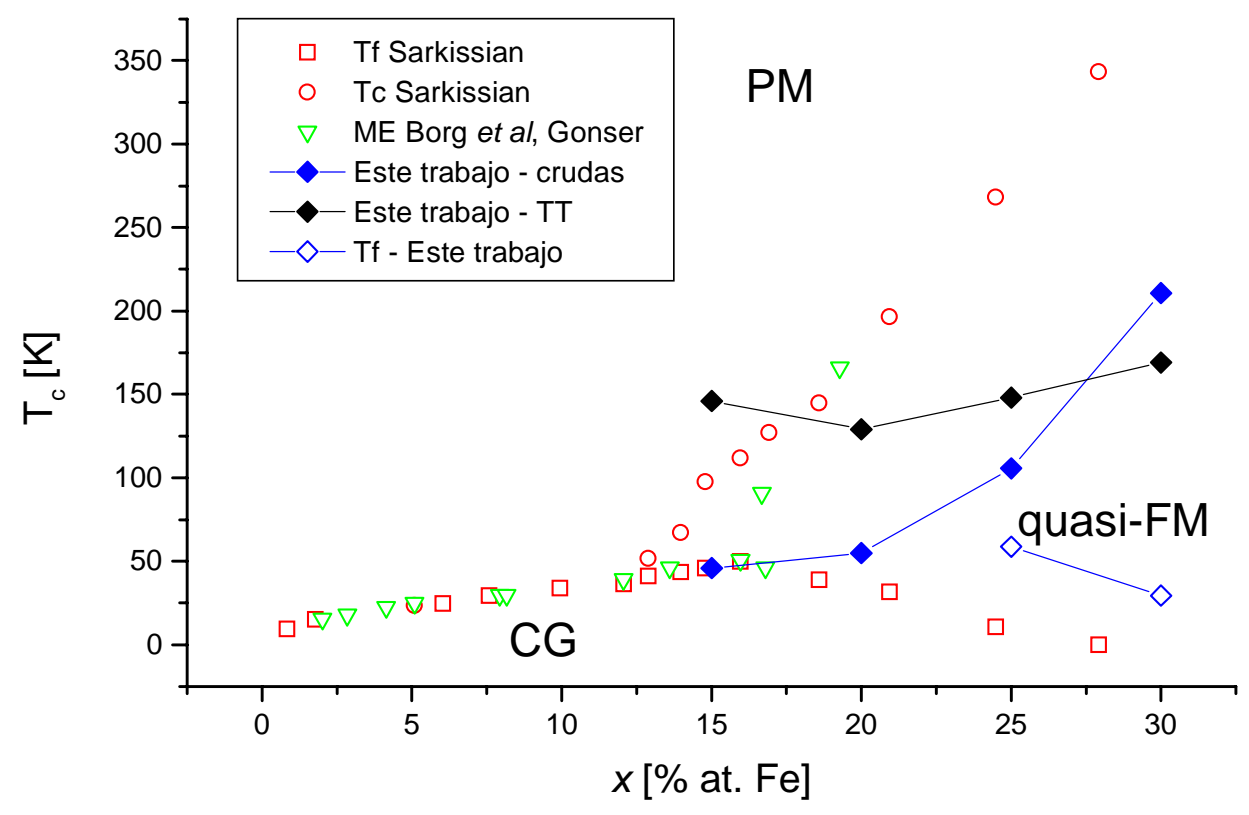

Fig. 18: Diagrama de fases magnéticas, según los datos reunidos por Coles y Sarkissian. Nuestros datos están agregados en azul (muestras crudas) y en negro (tratadas térmicamente). Los diamantes azules abiertos indican las temperaturas de reentrancia $\left(\mathrm{T}_{\mathrm{f}}\right)$, calculadas como contamos en §I.3.4. Indicamos las zonas

I.3.4. Con las muestras crudas procedimos de la siguiente manera: para las de $x=15 \mathrm{y}$ $20 \%$ calculamos la temperatura del máximo de la susceptibilidad; para las de $x=25 \mathrm{y}$ $30 \%$ utilizamos los criterios de Chien para ambas temperaturas: la de "hombro"o reentrancia como la intersección de dos rectas, y la $\mathrm{T}_{\mathrm{c}}$ como el punto de inflexión del lado derecho de la curva de susceptibilidad. La temperatura crítica de nuestra muestra $\mathrm{Fe}_{15} \mathrm{Au}_{85}$ se ubica en el mismo lugar que la de los otros autores (fig. 18). Las muestras de concentración superior quedan por debajo de la línea de temperaturas críticas, aunque siguen la tendencia ascendente de sus pares producidas por otro método. No nos 
es posible calcular un umbral de percolación como en el caso del Fe-Cu debido a que tenemos sólo dos puntos experimentales como para ajustar una curva. Todavía más, como dijimos anteriormente, la muestra con $x=25 \%$ no tiene un comportamiento claro como para adscribirla a una u otra rama del diagrama T- $x$.

En la literatura acerca de muestras Fe-Au obtenidas por enfriado rápido, es decir, supuestamente más aleatorias que las nuestras, se acepta que si se realiza trabajo mecánico sobre éstas hay una promoción de la clusterización. En nuestro caso, como el efecto del aleamiento mecánico es "bombear" a los átomos de Fe en la matriz del Au, y por lo ya comentado al comienzo de esta discusión, la aleación tendrá menos aleatoriedad que las hechas por enfriado rápido. Nuestras muestras tenderán a ser más clusterizadas que las antedichas, y por tanto tendrán menos átomos de Fe entre racimos. La interacción dominante entre racimos (la RKKY y/o la dipolar), se mantendrá hasta concentraciones más altas. Así, el umbral de percolación se corre a la derecha del diagrama de fases magnéticas, en relación a las muestras de los otros trabajos (fig. 18).

El tratamiento térmico sobre nuestras muestras da como resultado un aumento del efecto MRG, debido a la eliminación de defectos y no a cambios en la respuesta magnética ( $\Delta \rho$ prácticamente no cambia). Es posible que haya segregación de $\alpha$-Fe, como discutimos en §III.4.3.

Calculamos las temperaturas críticas de las muestras tratadas térmicamente. Vemos que tienen un comportamiento levemente creciente con la concentración, salvo $\mathrm{Fe}_{15} \mathrm{Au}_{85}$. De tal manera, la $\mathrm{T}_{\mathrm{c}}$ es menor en la muestra tratada que en la cruda para $x=$ $30 \%$. Un descenso de la $\mathrm{T}_{\mathrm{c}}$ similar a este puede ser observado en la figura 1 del trabajo de Griffith y Claus ([Griffith 1986]), donde por aplicación de mayores temperaturas en el tratamiento térmico a una muestra de $\mathrm{Fe}_{15} \mathrm{Au}_{85}$, ésta se "clusteriza" y el punto de 
inflexión se desplaza a temperaturas menores. Es posible que al tratar térmicamente a las muestras los clusters originales que estaban compuestos de hierro en una aleación desordenada con el Au, se "refinen", en el sentido de que se enriquece su contenido de hierro, al punto tal de transformarse a $\alpha$-Fe. Este $\alpha$-Fe puede estar como superparamagneto (si su tamaño de grano es muy pequeño), y por tanto sería responsable de alguno de los picos observados en la susceptibilidad. Sin embargo, se mantiene una componente que es la aleación original Fe-Au. No podemos dilucidar quién es el responsable del efecto magnetoresistivo: si es la aleación $\mathrm{Fe}-\mathrm{Au}$, el $\alpha$-Fe, o ambos al mismo tiempo. La componente ferromagnética (la curva sobre la que parecen estar "montados" los picos de la susceptibilidad) puede deberse a la aleación Fe-Au, o a la precipitación de otro compuesto ${ }^{11}$. Desafortunadamente no hemos contado con la ayuda de la espectroscopía Mössbauer para entender completamente el efecto del tratamiento térmico.

\footnotetext{
${ }^{11}$ Como ocurrió en el sistema Fe-Cu, donde precipitaron $\gamma$-Fe y carburos además del $\alpha$-Fe.
} Véase el capítulo II. 


\section{Conclusiones}


En esta parte intentaremos resumir lo que hemos aprendido del comportamiento de estos dos sistemas, y del efecto que produce en ellos el tratamiento térmico. Si bien hay disparidad en la cantidad de resultados que hemos obtenido en los dos sistemas, creemos que podemos establecer algunas ideas comunes. Las conclusiones de cada sistema en particular ya fueron obtenidas en cada capítulo, por lo que no las repetiremos.

Hemos confirmado que es posible obtener sistemas magnetoresistivo gigantes por medio del aleamiento mecánico. Hemos observado que la razón MRG es mayor en los sistemas obtenidos por MA que en los obtenidos por otras vías. La curva de efecto magnetoresistivo vs concentración de hierro tiene la misma forma para ambos sistemas, con máximos ubicados en $x=20$ y 25 para el $\mathrm{Fe}_{\mathrm{x}} \mathrm{Cu}_{100-\mathrm{x}}$ y el $\mathrm{Fe}_{\mathrm{x}} \mathrm{Au}_{100-\mathrm{x}}$, respectivamente. No hemos visto saturación a los campos medidos.

Ambos forman soluciones sustitucionales que alteran el parámetro de red $a$, de una manera que lo aleja de una dilución simple como lo indicado por la ley de Vegard. Esta alteración es monótona con el aumento en concentración de hierro. El efecto MRG indica que a pesar de la variación en $a$ con la concentración, la integral de intercambio $J$ proporciona un carácter ferromagnético a los clusters ricos en Fe. Por la forma de producción, el hierro no se dispersa en forma completamente aleatoria, sino que forma clusters coherentes con la matriz fcc. Este efecto se ve reforzado por la entalpía de formación positiva que tienen ambos sistemas.

El tamaño de grano en ambos sistemas es muy pequeño, lo que hace que los clusters de hierro sean monodominio. Pero no se trata de sistemas superparamagnéticos clásicos, sino fuertemente interactuantes. Las temperaturas de Weiss $\Theta$ dan valores altos para casi todas las muestras, y siguen un comportamiento similar con la concentración, como podemos apreciar en la figura 1. Ambos muestran características de mictomagne- 
tos, con umbrales de percolación próximos a la concentración prevista por Mydosh para un ferromagneto, esto es $17 \%$. Del criterio utilizado por el mismo autor, el cálculo de $W$, vemos que el comportamiento de las temperaturas críticas con la frecuencia directora en la medida de susceptibilidad es similar al de un vidrio de espín.

En este punto se nos ha hecho difícil deslindar si estamos tratando con superparamagnetos que hacen una transición SPM-PM o de un mictomagneto. Nos inclinamos a pensar que estamos frente a un sistema donde al ser las interacciones fuertes, estemos en la región próxima a la línea crítica $\mathrm{t}_{\mathrm{p}}$ mostrada por Mørup ([Mørup 1994]), donde ambos

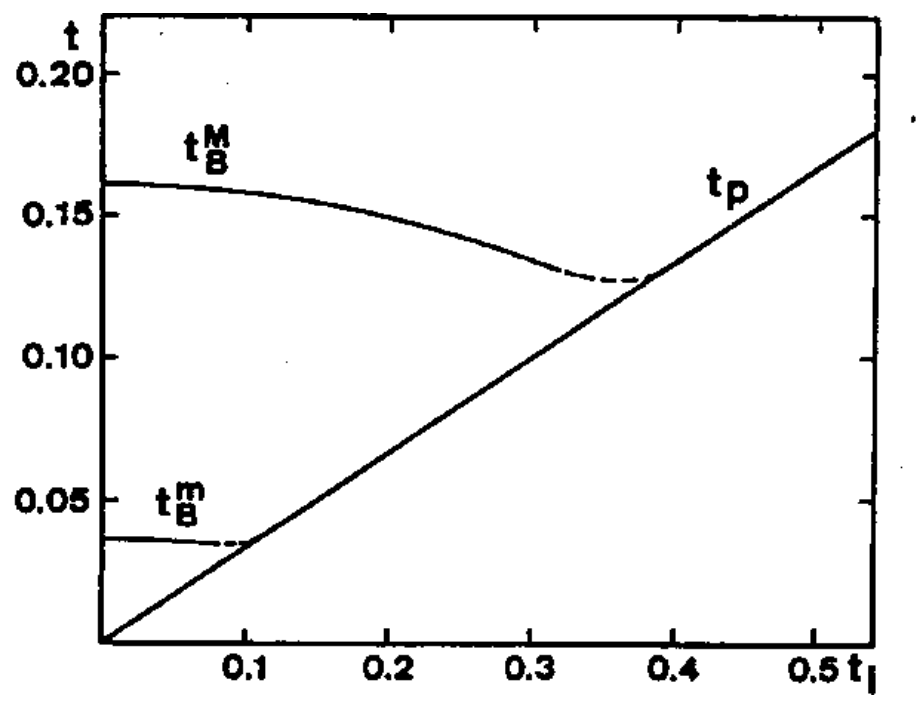

Fig. 1: Diagrama de fases que ilustra el carácter de las nanopartículas en función de la intensidad de la interacción. La parte con $t<t_{p}$ es la zona de vidrio de espín y la zona $t>t_{p}$ es la zona superparamagnética. Utilizando un modelo muy simplificado, se definen la temperatura reducida $t=k T / K V$, con $k$ la constante de Boltzmann, $K$ la constante anisotrópica y $V$ el volumen de la partícula; y la intensidad reducida de la interacción $t_{i}=\left\langle E_{i}{ }^{2}\right\rangle^{1 / 2} / K V$, con $E$ la energía de la interacción. La temperatura reducida de orden es $t_{p}=k T_{p} / K V, y$ $t_{B}^{m}=k T_{B}^{m} / K V$ y $t_{B}^{M}=k T_{B}^{M} / K V$ las temperaturas reducidas de bloqueo medidas por magnetización y Mössbauer, respectivamente.Tomado de [Mørup 1994].

comportamientos son indiscernibles por una medida física. Una forma de confirmar esto sería encontrar por susceptibilidad a las $\mathrm{T}_{\mathrm{c}}$ obtenidas por Mössbauer y viceversa, y ver 
si la relación entre temperaturas se aproxima a 1. En una muestra $\mathrm{Fe}_{40} \mathrm{Cu}_{60}$ preparada por $\mathrm{MA}^{1}$ se ve que esta relación es de 0,9 .

En ambos sistemas el efecto magnetoresistivo $\Delta \rho / \rho$ y la magnetoresistividad $\Delta \rho$ son mayores que en los sistemas producidos por condensación desde el estado gaseoso. Las explicaciones a esta diferencia pueden estar tanto en que los clusters producidos por un método tienen una combinación tamaño / momento magnético / distancia entre clusters más efectiva que el otro para dar un mejor efecto MRG o bien que la rugosidad de unos y otros clusters es diferente. Creemos que esto último es la causa, ya que es probable que los clusters producidos por aleamiento mecánico sean más rugosos que los producidos por condensación. Esto es factible debido a que el método de condensar desde el vapor parece producir clusters de forma más homogénea. Aunque no hemos hecho otra determinación de tamaño que por SAXS, los tamaños obtenidos $(\approx 2 \mathrm{~nm})$ son del orden de los obtenidos por sputtering.

\footnotetext{
${ }^{1}$ Datos tomados de [Monteiro 1998] y comunicación personal con el Dr. Larica. Ver diagrama de fases de §l.3.4
} 


\section{Apéndice A}

\section{Formación de carburos}

\section{Formación de carburos}

Hemos efectuado una serie de experimentos que nos han permitido entender la formación de carburos de hierro en la aleación Fe-Cu. Como hemos visto, la aparición de los carburos está ligada al uso de alcohol como agente antiadherente. La extrema ductilidad del cobre hace que en una molienda común el material quede completamente adherido a las paredes del recipiente y a las bolas. El uso de alcoholes en pequeña cantidad permite que el sistema se mezcle, debido a que el uso del agente antiadherente inhibe parcialmente al soldado en frío. Con la inhibición parcial, el balance entre fractura de las partículas y el soldado en frío de las mismas se mantiene [Lu 1999]. Si no se inhibiese este proceso de soldado, las partículas seguirían creciendo hasta unirse todas y pegar el material a las paredes del envase o formar una masa única. Por tanto, gracias al agente antiadherente (o Process Control Agent) el proceso de aleamiento mecánico pue- 
de continuar avanzando. Esta es una práctica usual en esta área del aleamiento mecánico ${ }^{1}$. Pero, como también hemos visto, en el sistema aparecen carburos de hierro luego del tratamiento térmico. Entendemos que esto se debe a la presencia de carbono en el

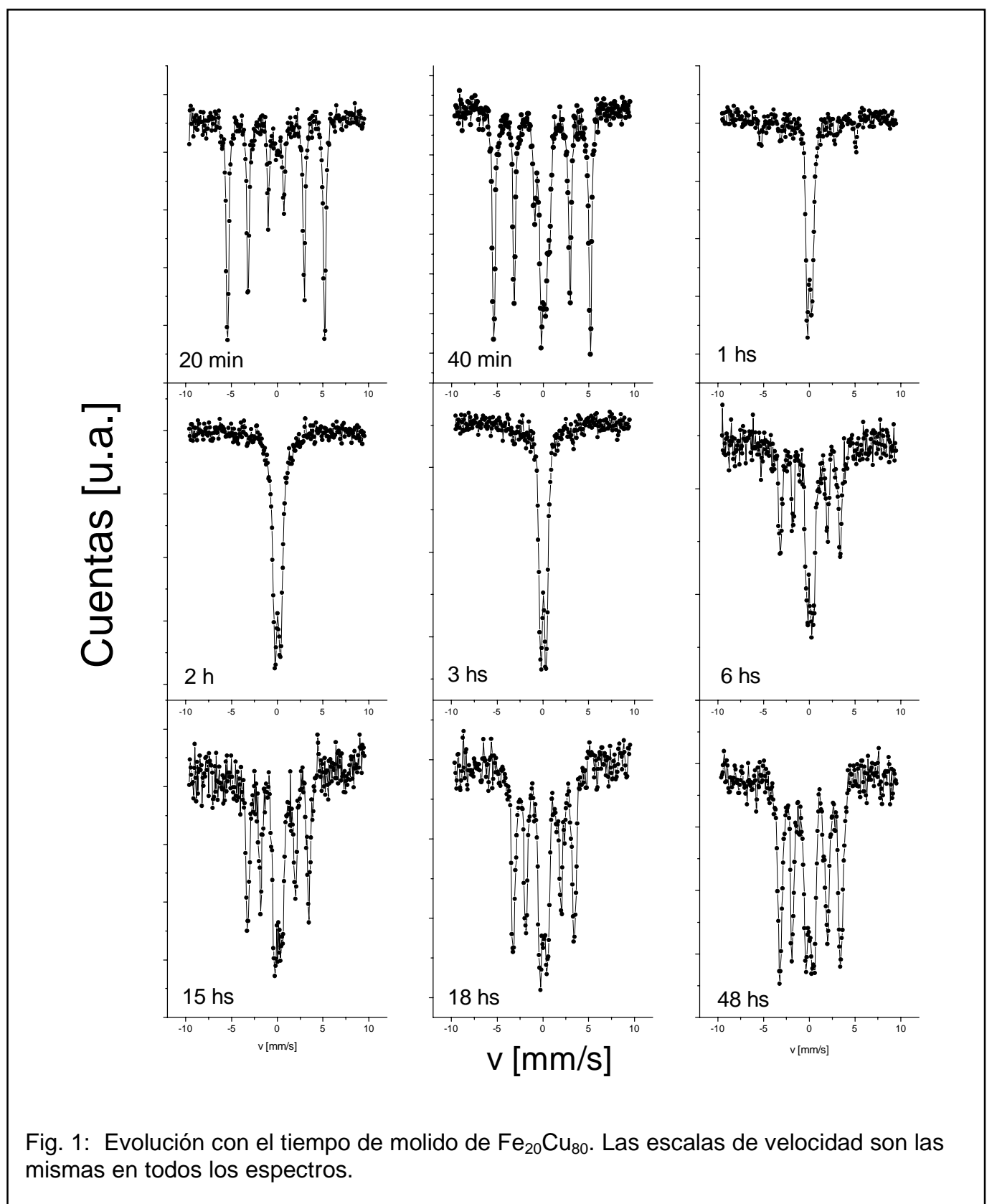

alcohol. El alcohol se disocia, tal vez parcialmente, con el trabajo mecánico y la elevación de la temperatura en el entorno de la zona de impacto. En estas zonas, la tempera-

\footnotetext{
${ }^{1}$ Ver en [Shingu 1991] los artículos de Uenishi et al. (pág. 459), Eckert et al. (pág. 505), Kuyama et al. (pág. 521), Morris et al. (pág. 529), Schwarz et al. (pág. 595), Suzuki et al. (pág. 639)
} 
tura local puede alcanzar $\operatorname{los} 100^{\circ} \mathrm{C}$, a pesar que el recipiente se mantiene prácticamente a temperatura ambiente. Esa temperatura es más grande que las de disociación del metanol o el benceno, que son de 64,6 y $80,1{ }^{\circ} \mathrm{C}$, respectivamente.

En nuestras primeras moliendas hemos utilizado metanol (fórmula $\mathrm{CH}_{3} \mathrm{OH}$ ) y también benceno (fórmula $\mathrm{C}_{6} \mathrm{H}_{6}$ ), ambos con similares resultados.

En esta parte hemos utilizado $0,3 \mathrm{cc}$ de metanol en mezclas de un total de $0,5 \mathrm{~g}$ de polvos de $\mathrm{Fe}$ y $\mathrm{Cu}$ de gran pureza, en proporción atómica 20:80. Hemos cerrado el cilindro cargado con la mezcla bajo atmósfera inerte (argón) y molido en un molino horizontal Retsch MM2000 con cilindro modificado. La relación de masas bola/material fue de 14. Variamos los tiempos de molido entre 20 minutos y 48 horas. Medimos las muestras por espectroscopía Mössbauer. Como podemos apreciar en la fig 1, para los tiempos iniciales de molido, el $\alpha$-Fe coexiste con un doblete. La proporción entre ellos va cambiando con el tiempo de molido, hasta llegar a que el espectro sólo consiste del doblete $(\Delta=0,58 \pm 0,01 \mathrm{~mm} / \mathrm{s}$ y $\delta=0,16 \pm 0,01 \mathrm{~mm} / \mathrm{s})$. Esto ocurre a las dos horas de trabajo mecánico. En ese tiempo, el campo del sextete se va reduciendo ligeramente, desde los $33 \mathrm{~T}$ correspondientes al $\alpha$-Fe en bloque, hasta $\mathrm{B}_{\mathrm{hf}}=32,27 \mathrm{~T}$ para $\mathrm{t}=1 \mathrm{~h}$. A las tres horas se empieza a esbozar un sextete algo ensanchado, con un campo hiperfino $\mathrm{B}_{\mathrm{hf}}=20 \mathrm{~T}$. Con el correr del tiempo, este sextete se hace dominante hasta alcanzar un $76 \%$ del espectro (para 48 horas de molido), y el campo crece ligeramente hasta $\mathrm{B}_{\mathrm{hf}}=$ $20,71 \pm 0,34 \mathrm{~T}$, con un corrimiento isomérico $\delta=0,21 \pm 0,01 \mathrm{~mm} / \mathrm{s}$. Este sitio tiene parámetros muy similares a los reportados en [Le Caër 1976] para la cementita $\left(\mathrm{Fe}_{3} \mathrm{C}\right)$. El área restante corresponde al doblete ya mencionado, que mantiene sus parámetros $(\Delta=$ $0,52 \pm 0,2 \mathrm{~mm} / \mathrm{s}$ y $\delta=0,16 \pm 0,02 \mathrm{~mm} / \mathrm{s})$. Observemos que los parámetros hiperfinos 
del doblete son similares a los del $\mathrm{Fe}_{20} \mathrm{Cu}_{80}$ del cuerpo principal de esta tesis $(\Delta=0,54 \pm$ $0,01 \mathrm{~mm} / \mathrm{s}$ y $\delta=0,17 \pm 0,01 \mathrm{~mm} / \mathrm{s})$.

Por DRX observamos que los picos del Cu se ensanchan, a la vez que los picos del Fe desaparecen. No es posible detectar la presencia del carburo de hierro en los difractogramas, excepto hasta transcurridas 48 horas de molido, donde aparece una pequeña protuberancia al lado del pico (111) del $\mathrm{Cu}$. De este hecho podemos decir que la cementita se encuentra en zonas de poco volumen, con un número insuficiente de parámetros de red como para producir una intensidad de difracción lo suficientemente localizada como para ser fácilmente detectada por DRX.

Podemos inferir que la formación de la cementita o similares se produce en un paso posterior a la formación del doblete. Los carburos de hierro, en especial la cementita, son bastante estables. Pensamos que el carbono sólo puede encontrarse parcialmente en los gránulos magnéticos (posiblemente en las interfaces con la matriz más rica en $\mathrm{Cu}$ ), y en forma intersticial. Sólo el trabajo mecánico adicional (en este caso, sólo luego de las tres horas de molido) forma al carburo. Así, podemos afirmar que las muestras investigadas son una solución sólida Fe-Cu. El trabajo mecánico posterior hace que se formen carburos de hierro.

Adicionalmente, podemos decir que el molino horizontal Retsch utilizado en esta parte parece ser más energético que el vibratorio Gikken utilizado para fabricar las muestras de la parte principal de esta tesis. 


\section{Apéndice B}

\section{Dispersión de rayos $\mathrm{X}$ a bajos ángulos (SAXS)}

\section{Dispersión de rayos $\mathrm{X}$ a bajos ángulos}

La dispersión de rayos $\mathrm{X}$ a bajos ángulos, o SAXS (Small Angle X-ray Scattering) es una técnica basada en analizar la dispersión de rayos $\mathrm{X}$ producida por un material al paso del haz, a ángulos muy próximos a cero.

Cualquier evento de dispersión está caracterizado por una ley recíproca entre tamaño de partícula y ángulo de dispersión ([Kratky 1982]). La radiación electromagnética incidente interactúa con los electrones en una muestra. Una parte de ellos emitirá radiación coherente. Donde las ondas interfieren constructivamente tendremos un máximo, que es lo que detectamos. El máximo de la intensidad estará en la dirección de $2 \theta$ (siendo $\theta$ el ángulo de incidencia). Consideremos un objeto de longitud del orden del angstrom. La interferencia constructiva ocurrirá cuando la diferencia de camino sea igual a una longitud de onda $\lambda$. Para un objeto de tamaño 
mayor, la interferencia constructiva de las ondas producidas por dos electrones ubicados en extremos opuestos del objeto ocurrirá para un ángulo $2 \theta$ menor que antes, ya que la diferencia de camino es menor (ver figura 1). Los objetos que tengan dimensiones características del orden de los nanómetros mostrarán máximos a ángulos muy pequeños ([Porod 1982]). La forma de la curva de dispersión puede ser calculada si conocemos la forma del

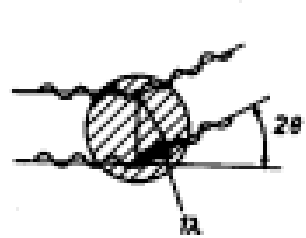

(a)

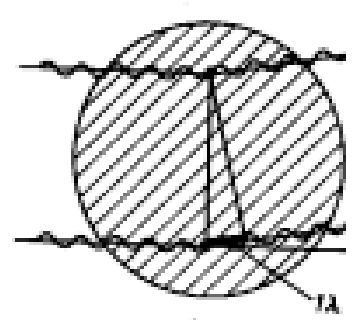

(b)

FIG. 1

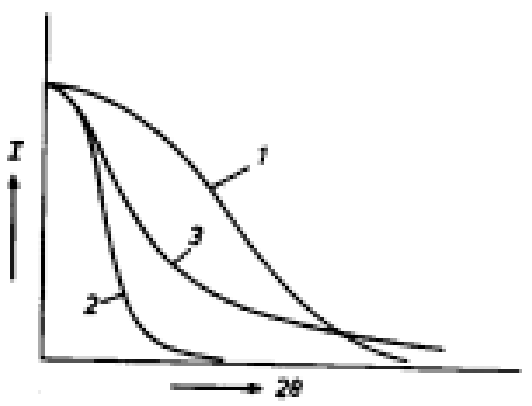

Fig. 1: una onda incidente incidiendo sobre dos esferas de diferente tamaño. La esfera de (a) producirá la curva de dispersión 1 del gráfico inferior. La esfera mayor (b) producirá la curva 2. Tomado de [Kratky 1982] pág. 4.

ente dispersante. Si se conoce la función distribución de electrones $p(r)$ en el ente dispersante, la curva de dispersión será:

$$
I(k)=4 \pi \int_{0}^{\infty} p(r) \frac{\operatorname{sen} k r}{k r} d r
$$

donde $k=4 \pi \frac{\operatorname{sen} \theta}{\lambda}$.

Para partículas pequeñas consideramos que tienen densidad electrónica uniforme.

Cuando tratamos con materiales reales debemos considerar que nuestro ente dispersante está inmerso en otro. Es por eso que la densidad electrónica que nos dará la curva de dispersión es la diferencia entre las densidades de los dos medios. Si no existiese diferencia entre las densidades, no tendríamos dispersión. A esta densidad electrónica efectiva se la suele llamar "contraste". 
En los sistemas muy diluídos el análisis es sencillo, ya que las intensidades producidas por cada ente dispersante simplemente se suman. No es ese el caso donde los sistemas presentan heterogeneidades, o son más densos. El problema del SAXS es deducir forma, tamaño, masa y densidad electrónica de un sistema a partir de una curva de dispersión. Uno debe proponer un modelo que pueda describir al sistema y que su curva de dispersión coincida con la obtenida experimentalmente.

Una magnitud relevante en el análisis de un experimento SAXS es el invariante Q, definido como: $Q=\int_{0}^{\infty} k^{2} I(k) d k$, que permanece constante ante deformaciones o corrimientos en el sistema ${ }^{1}$

La forma de la curva de dispersión tiene dos zonas características: la de Guinier, que está ubicada en la zona de pequeños valores de $k$, y la de Porod, que está ubicada para valores de $k \rightarrow \infty$. La curva de dispersión de todo ente dispersante sigue una ley $k^{-4} \mathrm{a}$ grandes valores de $k$.

De una fuente de luz sincrotrón es posible obtener un haz de rayos $\mathrm{X}$ monocromático, bien colimado, puntual e intenso, cualidades que lo hacen inmejorable para hacer medidas a bajos ángulos. Otra ventaja adicional es que se puede cambiar la longitud de onda con facilidad. Hemos utilizado para nuestras medidas la estación SAXS del Laboratorio Nacional de Luz Síncrotron ${ }^{2}$.

\footnotetext{
${ }_{2}^{1}$ [Porod 1982] pág 22

2 Ubicado en Campinas, Brasil. Más detalles sobre esta línea se pueden encontrar en [Kellerman 1997].
} 


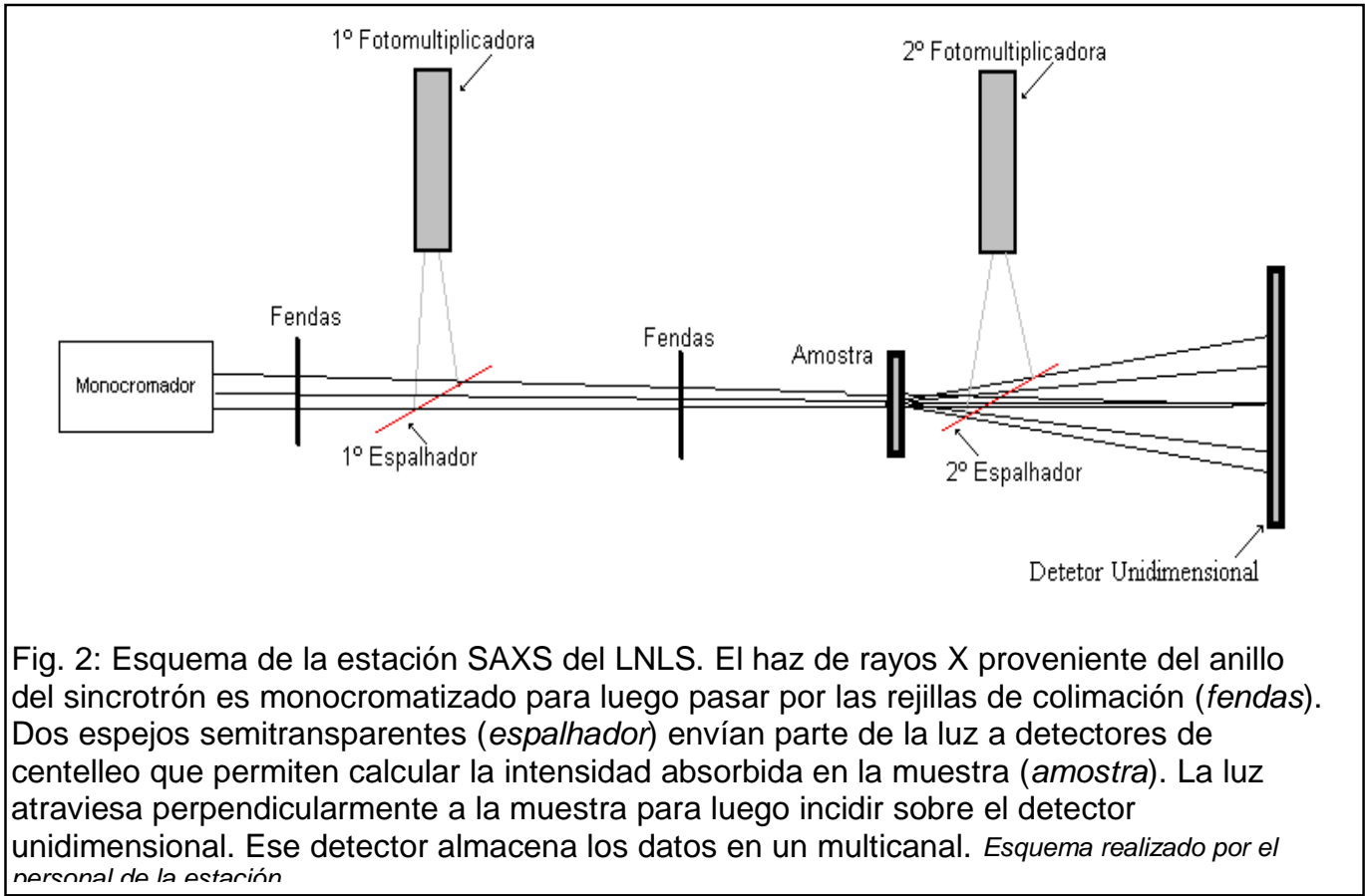

Utilizamos longitudes de onda por debajo de los bordes de absorción de los elementos presentes en la muestra, ya que la amplitud de scattering $f$ depende $^{3}$ de la longitud de onda de los rayos X:

$$
f(\lambda)=f_{0}+f^{\prime}(\lambda)+i f^{\prime \prime}(\lambda)
$$

donde $f^{\prime}$ y $f$ "' son las correcciones a la amplitud de scattering y son conocidas como los factores de scattering anómalo. Ambas son significativas en proximidades de las longitudes de onda correspondientes a los bordes de absorción

\section{Tratamiento de los datos}

En un experimento de SAXS obtenemos el número de cuentas detectado en función del canal. Estos datos obtenidos tienen que ser tratados para poder ser analizados. Al paso del haz de rayos X por la muestra se produce una absorción que debe ser tenida en cuenta. Para separar la intensidad que efectivamente interactúa en forma coherente con los electrones de la muestra normalizamos los datos dividiendo por 
$I_{T} h e^{-\mu d}$, donde $h$ es la respuesta del detector para cada canal, $\mu$ es el coeficiente de absorción, $d$ es el espesor de la muestra, $I_{T}$ es la intensidad integrada al tiempo de adquisición. Evaluamos el factor exponencial detectando la intensidad antes y después de la muestra con centelladores (fig.2). Restamos la dispersión parásita usando una medida registrada en las mismas condiciones pero sin muestra. Además, removimos la contribución constante causada por la dispersión incoherente (que se produce por fluorescencia o por dispersión Raman resonante). Para ello usamos la ley de Porod, que establece que a $k$ grandes, la intensidad decrece según $k^{-4}$. Ajustamos cada espectro (en el rango $0,1 \leq k \leq 0,20 \AA^{-1}$ ) con:

$$
A+B / k^{4}
$$

De esta manera obtuvimos el valor de $A$, que sustrajimos del espectro.

${ }^{3}$ [Feigin 1987] pág. 16 


\section{Apéndice $\mathbf{C}$}

\section{Susceptómetro CA y SQUID}

\section{Susceptómetro CA}

El principio básico de funcionamiento de un susceptómetro de corriente alterna (CA) es que la inductancia de una bobina cambia si le acercamos un material. Por la ley de Faraday, un cambio en el flujo magnético que baña un circuito eléctrico producirá una fuerza electromotriz (fem). Este cambio se debe a las propiedades magnéticas del material. El arreglo que se usa generalmente consta de dos bobinas coaxiales exactamente iguales, bobinadas en sentido contrario. Ambas, llamadas bobinas secundarias, se montan una encima de la otra. El conjunto formado por ambas bobinas se ubica en el interior de otra bobina, llamada primaria, también coaxial (ver figura 1). En esta situación, si producimos un campo magnético mediante la excitación del bobinado primario con una corriente alterna de frecuencia $f$, las fem inducidas en las bobinas secundarias se cancelarán. Al colocar nuestra muestra en el eje de las bobinas, la inductancia cambia, 
siendo este cambio directamente proporcional a la susceptibilidad de la muestra. Si colocamos un material en el interior de alguna de las dos bobinas detectaremos un cambio en la fem inducida que es directamente proporcional a la susceptibilidad dinámica $\chi_{\mathrm{d}}$ del material (la fem inducida en la otra bobina resta la fem correspondiente a la bobina en donde se encuentra el

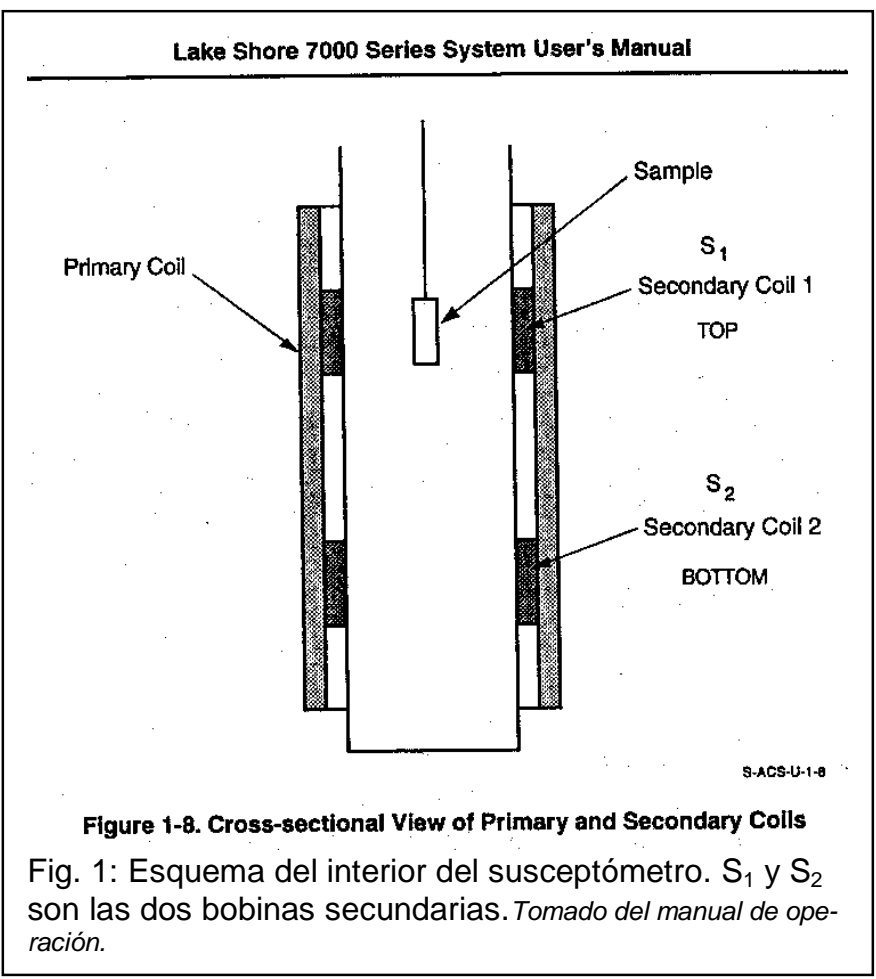

material). Si movemos la muestra desde el centro de una de las bobinas secundarias al centro de la otra cancelamos todo efecto externo al material, como las desigualdades físicas entre ambas bobinas o campos externos parásitos. Para obtener la susceptibilidad a partir de la tensión medida en las bobinas secundarias solamente necesitamos saber el factor de proporcionalidad $\alpha$, y eso se obtiene midiendo un paramagneto de susceptibilidad conocida, o bien utilizando una expresión matemática. La lectura de tensión se convertirá en la medida de la susceptibilidad mediante:

$$
\chi=\alpha v / m f H
$$

donde $v$ es la tensión medida, $m$ la masa de la muestra, $f$ la frecuencia de excitación, y $H$ la amplitud media cuadrática del campo externo.

Este dispositivo es eficaz aún a frecuencias elevadas (hasta $50 \mathrm{kHz}$ ). El dispositivo es capaz de detectar la susceptibilidad en fase $\left(\chi^{\prime}\right)$ y fuera de fase $\left(\chi \chi^{\prime}\right)$. Normalmente es muy útil medir la respuesta de un material cuando cambia la temperatura, y se lo- 
gra esto sumergiendo el conjunto en un termo al alto vacío, en baño frío de helio. Un elemento calefactor permitirá trabajar en un rango de temperaturas de entre pocos $\mathrm{K}$ a unos $300 \mathrm{~K}$.

El aparato permite dos modos de operación diferenciados: en rampa y por lista. En el primero se le indica al software de control que eleve la temperatura a tasas fijas (PE $2 \mathrm{~K} / \mathrm{min}$ ). En este modo el aparato comenzará a medir no bien haya transcurrido el tiempo prefijado por la tasa y no esperará a estabilizar la temperatura para lograr ese cometido. Tiene como ventaja la rapidez con la que es posible lograr una medida (típicamente 4 horas) y como desventaja la menor precisión. El segundo modo, el de lista, hace que el aparato haga las medidas una vez que la temperatura se ha estabilizado dentro de un rango alrededor del valor deseado. Para una medida típica (con cuatro frecuencias directoras) hacen falta del orden de 20 horas.

\section{SQUID}

Son las siglas para "Superconductor Quantum Interference Device", y designa a un aparato que utiliza el efecto Josephson para la medida de la magnetización de una muestra. Esto se logra cortando un anillo superconductor con una lámina muy delgada de material aislante. Esta lámina constituye una "unión Josephson” con el anillo. El anillo superconductor por sí solo tiene un flujo estable producido por la supercorriente, insensible a los cambios externos. La lámina permite cambiar ese flujo en cantidades discretas. Como justamente esa lámina es la única parte del circuito que puede percibir flujos externos, obliga al anillo a cambiar el suyo, puesto que el flujo intentará mantenerse. Un solenoide arrollado sobre el anillo sensa esos cambios, transduciéndolos en una fem. De allí, un circuito electrónico completa la tarea de registrar la medida. 
El flujo en el anillo $\varphi$ será la suma del flujo externo $\varphi_{\mathrm{e}} \mathrm{y}$ del flujo debido a la supercorriente $\varphi_{s}$ :

$$
\varphi=\varphi_{e}+\varphi_{s}
$$

donde $\varphi_{\mathrm{s}}=L i_{s}$, con $L$ la inductancia del anillo e $i_{s}$ la supercorriente. La supercorriente estará determinada por las propiedades de la unión Josephson a través de:

$i_{s}=i_{c} \operatorname{sen} \theta$,

donde $i_{c}$ es la corriente crítica y $\theta$ la diferencia de fase de las funciones de onda electrónica al pasar a través de la unión.

De esta manera tenemos que el flujo en el anillo es una función

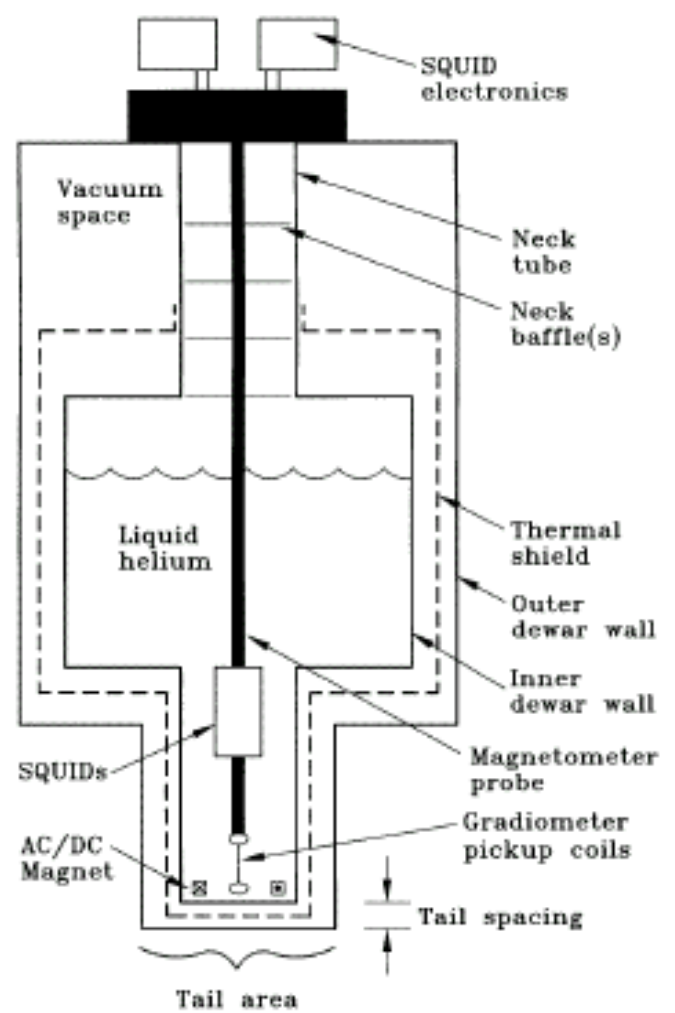

Fig. 2: Esquema de un SQUID de bajas temperaturas. Tomado de [Jiles 1996]

trascendente del flujo externo:

$\varphi=\varphi_{e}+L i_{c} \operatorname{sen} \theta$

El flujo en el anillo está cuantizado en unidades de $\varphi_{0}=h / 2 e=2,067 \times 10^{-15} \mathrm{~Wb}$, donde $h$ es la constante de Planck y $e$ la carga del electrón. De esta manera $\varphi=N \varphi_{0}$, donde $N$ es un entero. El ángulo de fase $\theta$ depende del flujo en el anillo de la siguiente manera: $\theta=2 \pi N-2 \pi\left(\varphi / \varphi_{0}\right)$, y como $N$ es un entero $\operatorname{sen} \theta=\operatorname{sen}\left(-2 \pi \varphi / \varphi_{0}\right) \Rightarrow$ $\varphi=\varphi_{e}-L i_{c} \operatorname{sen}\left(2 \pi \varphi / \varphi_{0}\right)$ 
Cada vez que el flujo en el anillo sea un múltiplo de $\varphi_{0}$ el seno se hará nulo, con lo que $\varphi=\varphi_{\mathrm{e}} \mathrm{y}$ el bobinado medirá una fem proporcional a ese flujo externo. Como $\varphi_{0}$ es una cantidad muy pequeña, cualquier flujo introducido en el anillo estará muy próximo a ser uno de sus múltiplos, y así la medida estará muy próxima a su valor real.

Los SQUID comerciales suelen estar construídos con un anillo de $\mathrm{Nb}$ o Yba$\mathrm{CuO}^{1}$, dependiendo de la temperatura de operación deseada (baja o alta). La lámina separadora suele ser una tricapa. Normalmente el conjunto se sumerge en un baño frío (He o $\mathrm{N}$ líquidos). La muestra se coloca en una zona próxima, entre los polos de un imán. Un elemento calefactor permite medir a diversas temperaturas.

${ }^{1}$ [Jenks 1997] 


\section{Referencias}

[Altbir 1996] D. Altbir, J. d'Albuquerque e Castro y P. Vargas; PRB 5410 (1996) 6823

[Ambrose 1993] T. Ambrose, A. Gavrin, C. L. Chien; JMMM 124 (1993) 15

[Baibich 1988] M. N. Baibich, J. M. Brotto, A. Fert, F. Nguyen Van Dau, F. Petroff, P. Etienne, G. Creuzet, A. Friedrich y J. Chazelas; Phys. Rev. Lett. 61 (1988) 2472

[Baibich 1996] M. N. Baibich en Magnetism, Magnetic materials and their applications, F. Leccabue y V. Sagredo, World Scientific, Singapur (1996) págs. 69 - 80

[Barro 1997] M. J. Barro, E. Navarro, P. Agudo, A. Hernando, P. Crespo y A. García Escorial; Mat. Sci. Forum 235-238 (1997) 553

[Beck 1983] P. A. Beck; Phys. Rev. B 285 (1983) 2516

[Beck 1985] P. A. Beck; Phys. Rev. B 3211 (1985) 7255

[Borg 1972] R. J. Borg, D.Y. F. Lai y C. E. Violet; PRB 53 (1972) 1035

[Bowen 1993] L. H. Bowen, E. De Grave y R. E. Vandenberghe; "Mössbauer effect studies of magnetic soils and sediments" en Mössbauer spectroscopy applied to magnetism and materials science, Vol. 1, G. J. Long y F. Grandjean (Eds.), Plenum, Nueva York (1993) pág. 115 y ss.

[Canella 1975] V. Cannella y J. A. Mydosh; PRB 611 (1975) 4220

[Cahn 1968] J. W. Cahn; Transactions of the metallurgical society of AIME, 242 (1968) 166

[Chien 1986] C. L. Chien, S. H. Liou, D. Kofalt, Wu Yu, T. Egami y T. R. McGuire, Phys. Rev. B 33 (1986) 3247

[Chien 1993] C. L. Chien, J. Q. Xiao y J. S. Jiang; J. Appl. Phys. 7310 (1993) 5309

[Childress 1991] J. R. Childress y C. L. Chien, PRB 4310 (1991) 8089

[Coles 1977] B. R. Coles, R. H. Taylor, B. V. B. Sarkissian, J. A. Khan y M. H. Bennet; Physica B, 86-88 (1977) 275

[Coles 1978] B. R. Coles, B. V. B. Sarkissian y R. H. Taylor; Phil. Mag. B 374 (1978) 489

[CRC 2000] CRC Handbook of Chemistry and Physics (CD-ROM version), D. R. Lide (editor), Chapman \& Hall / CRC (2000)

[Crespo 1993] P. Crespo, A. Hernando, R. Yavari, O. Drbohlav, A. García Escorial, J. M. Barandiarán e I. Orúe; Phys. Rev. B 4810 (1993) 7134

[Crespo 1994] P. Crespo, A. Hernando y A. García Escorial; Phys. Rev. B 4918 (1994) 13227.

[Crespo 1995] P. Crespo, A. Hernando y A. García Escorial; Mat. Sci. Forum 179-181 (1995) 225

[Chowdhury 1986] D. Chowdhury; Spin glasses and other frustrated systems, World Scientific (1986)

[de Boer 1989] F. R. de Boer, R. Boom, W. Mattens, A. R. Miedema, y A. Niessen; Cohesion in metals: Transition metal alloys, North Holland, Amsterdam (1989) p. 240

[Ding 1995] J. Ding, M. Eilon, R. Street, T. St. Pierre, P. Smith y P. G. McCormick, JMMM 140-144 (1995) 471. 
[Dupuis 1997] V. Dupuis, J. Tuaillon, B. Prevel, A. Perez, P. Melinon, G. Guiraud, F. Parent, L. B. Steren, R. Morel, A. Barthelemy, A. Fert, S. Mangin, L. Thomas, W. Wernsdorfer y B. Barbara; JMMM 165 (1997) $42-45$.

[Drbohlav 1994] O. Drbohlav y A. R. Yavari; JMMM 137 (1994) 243.

[Eckert 1992] J. Eckert, J. C. Holzer, C. E. Krill III y W. L. Johnson; J. Mater. Res. 78 (1992) 1980.

[Eckert 1993] J. Eckert, J.C. Holzer, y W. L. Johnson; J. Appl. Phys. 731 (1993) 131.

[Eilon 1995] M. Eilon, J. Ding y R. Street, J. Phys.: Condens. Matter 7 (1995) 4921.

[Elkalkouly 1995] R. Elkalkouli, P. Chartier y J. F. Dinhut; Mat. Sci. Forum 179-181 (1995) 267.

[Essam 1980] J. W. Essam; Percolation Theory ; en Rep. Prog. Phys. 43 (1980) pag. 53

[Fabricius 1994] G. Fabricius, Tesis Doctoral, FCE - UNLP (1994)

[Feigin 1987] L. A. Feigin y D. I. Svergun Structure Analysis by Small-Angle X-Ray and Neutron Scattering, G.W. Taylor (ed.), Plenum Press, Nueva York (1987)

[Ferrari 1999] E. F. Ferrari, F. C. S. da Silva y M. Knobel; PRB 5913 (1999) 8412

[Gerritsen 1951] A. N. Gerritsen y J. O. Linde; Physica 17 (1951) 584

[Gerritsen 1953] A. N. Gerritsen; Physica 19 (1953) 61

[Goya 2001] G. F. Goya, H. R. Rechenberg y M. R. Ibarra, presentación al ISMANAM 2001

[Griffith 1986] G. Griffith y H. Claus; JMMM 54-57 (1986) 151

[Hernando 1993] A. Hernando, P. Crespo, A. García Escorial, J. M Barandiarán; Phys. Rev. Lett 7022 (1993) 3521

[Huang 1994] J.Y. Huang, A. Q. He, Y. K. Wu; Nanostruct. Materials 41 (1994) 1

[Huang 1996] J. Y. Huang, A. Q. He, Y. K. Wu, H. Q. Ye y D. X. Li; J. of Mat. Sci. 31 (1996) 4165

[Huang 1998] J.Y. Huang, J. Z. Jiang, H. Yasuda y H. Mori; PRB 5818 (1998) 11817

[Jenks 1997] W. G. Jenks, S. S. H. Sadegh y J. P. Wikswo Jr.; J. Phys. D: Appl. Phys. 30(1997) 293-323

[Jiang 1993] J. Z. Jiang, U. Gonser, C. Gente y R. Bormann; Appl. Phys. Lett. 6320 (1993) 2768

[Jiles 1996] D. Jiles, Introduction to Magnetism and Magnetic Materials, Chapman \& Hall, Londres (1996)

[Kaloshkin 1998] "Phase transformations and hyperfine interactions in mechanically alloyed Fe-Cu solid solutions", S. D. Kaloshkin, I. A. Tomilin, G. A. Andrianov, U. V. Baldokhin y E. V. Shelekhov; presentación al International Symposium on Metastable, Mechanically Alloyed, Nanocrystalline Materials; Wollongong, 1998

[Kellerman 1997] G. Kellerman, F. Vicentin, E. Tamura, M. Rocha, H. Tolentino, L. Barbosa, A. Craievich e I. Torriani; J. Appl. Cryst. 30 (1997) 1-4

[Klug 1974] H. P. Klug y L.E. Alexander; X-ray diffraction procedures, John Wiley \& Sons, Nueva York (1974) pág. 661

[Kratky 1982] O. Kratky, en Small-Angle X-ray Scattering, O. Glatter y O. Kratky, (eds.), Academic Press, Londres (1982) pág. 3 
[Kuhrt 1993] C. Kuhrt y L. Schultz; IEEE Transactions on Magnetics 296 (1993) 2667 [Le Caër 1976] G. Le Caër, J. M. Dubois y J. P. Senateur; J. of Solid State Chemistry [Levy 1994] P. M. Levy; "Giant magnetoresistance in magnetic layered and granular materials" en Solid state physics, vol. 47, Academic Press, San Diego (1994) pág. 367 y ss.

[Liu 1978] S. Liu y J. Moteff; J. Appl. Cryst. 11 (1978) 597

[Lu 1999] L. Lu e Y. F. Zhang; J. of Alloys and Compounds 290 (1999) 279

[Ma 1993] E. Ma, M. Atzmon, F. E. Pinkerton; J. Appl. Phys. 742 (1993) 955

[Ma 1997] E. Ma, J. H. He y P. J. Schilling; PRB 559 (1997) 5542

[Macrí 1994] P. P. Macrí, P. Rose, R. Frattini, S. Enzo, G. Principi, W. X. Hu y N. Cowlam; J. Appl. Phys. 767 (1994) 4061

[Macrí 1995a] P. P. Macrí, P. Rose, D. E. Banda, N. Cowlam, G. Principi y S. Enzo; Mat. Sci. Forum 179-181 (1995) 249.

[Macrí 1995b] P. P. Macrí, S. Enzo, N. Cowlam, R. Frattini, G. Principi y W. X. Hu; Phil. Mag. B71 2 (1995) 249.

[Massalski 1990] T. M. Massalski (comp.), Binary alloy phase diagram, ASM International, Materials Park (1990)

[Mazzone 1996] G. Mazzone y M. Vittori Antisari; PRB 541 (1996) 441

[Mørup 1980] S. Mørup, "Magnetic microcrystals", en Applications of Mössbauer spectroscopy, Vol II, editado por R. L. Cohen, Academic Press, New York (1980) pág. 1

[Mørup 1994] S. Mørup; Europhys. Lett. 28 (9) (1994) 671-676

[Monteiro 1998] D. W. L. Monteiro, C. Larica, E. Nunes, E. C. Passamani y K. M. B. Alves; Hyperfine Interactions (C) 3 (1998) 17-20

[Mydosh 1993] J. A. Mydosh, en Spin glasses; Taylor \& Francis eds. (1993)

[Nakhimovitch 1941] N. M. Nakhimovitch; J. Phys. USSR 5 (1941) 141

[Nigam 1983] A. K. Nigam y A. K. Majumdar; PRB 271 (1983) 495

[Pochet 1995] P. Pochet, PRB 52 (1995) 4006

[Porod 1982] G. Porod, en Small-Angle X-ray Scattering, O. Glatter y O. Kratky, (eds.), Academic Press, Londres (1982) pág. 35

[Pureur 1999] P. Pureur y C. M. Haetinger; presentación a la Escola Brasileira de Magnetismo 1999

[Raap 1995] M. B. Fernández van Raap, M. J. Regan y A. Bienenstock; J. of Noncrystalline Solids 191 (1995) 155-163

[Rohrer 1968] H. Rohrer; Phys. Rev. 1742 (1968) 583

[Ron 1980] M. Ron; "Iron-Carbon and Iron-Nitrogen systems" en Applications of Mössbauer spectroscopy, Vol II, editado por R. L. Cohen, Academic Press, New York (1980) pág. 329

[Rossiter 1991] P. L. Rossiter; The electrical resistivity of metals and alloys, Cambridge University Press, New York (1991)

[Roth 1977] M. Roth; J. Appl. Cryst. 10 (1977) 172

[Sánchez 1998] F. H. Sánchez, C. E. Rodríguez Torres, M. B. Fernández Van Raap y L. A. Mendoza Zélis; Hyp. Interact. (C) 269-277 (1998) 113 
[Sarkissian 1981] B. V. B. Sarkissian; J. Phys. F: Metal Phys. 208 (1981) 2191

[Saxena 1994] S. S. Saxena, J. Tang, Y. Lee y C. J. O'Connor; J. Appl. Phys. 7610 (1994) 6820.

[Schilling 1996] P. J. Schilling, J. H. He, J. Cheng y E. Ma; Appl. Phys. Lett. 686 (1996) 767

[Schwarz 1998] R. B. Schwarz, Mat. Sci. Forum, Vols. 269 - 272 (1998) 665.

[Shenoy 1978] G. Shenoy y F. Wagner, Mössbauer isomer shifts, North Holland, Amsterdam (1978) pág. 409

[Shingu 1990] P. H. Shingu, K. Ishihara, K. Uenishi, J. Kuyama, B. Huang y S. Nasu; en Solid state powder processing, A. H. Clauer y J. J. deBarbadillo (eds.), The Minerals, Metals \& Materials Society, (1990) pág. 21

[Shingu 1991] P.H. Shingu (editor), Mechanical alloying, Proceedings del I ${ }^{\text {st }}$ International Symposium on Metastable, Mechanically Alloyed, and Nanocrystalline Materials; Kyoto 1991; Materials Science Forum Vol. 88-90, Singapur (1992)

[Shingu 1999] P.H. Shingu, K. N. Ishihara, A. Otsuki, M. Hashimoto, N. Hasegawa, I. Daigo, y B. Huang; Mat. Sci. Forum, 312-314, (1999) 293

[Svergun 1992] D. I. Svergun; J. Appl. Cryst. 25 (1992) 495

[Sumiyama 1983] K. Sumiyama e Y. Nakamura; JMMM 35 (1983) 219-220

[Tie 1995] L. Tie, L. Yu-zhi, Z. Yu-heng, G. Chen, W. Shi-quiang y L. Wen-han; PRB 522 (1995) 1120

[Ueda 1996] Y. Ueda, S. Ikeda, S. Moriwaki y M. Matsuda, Jpn. J. Appl. Phys. 35 parte $2 \mathrm{~N}^{\circ} 8 \mathrm{~B}$ (1996) L1059

[Uenishi 1992] K. Uenishi, K. Kobayashi, S. Nasu, H. Hatano, K. Ishihara y P.H.

Shingu, Z. Metallkd. 83 (1992) 132

[Wang 1994] J. Q. Wang y G. Xiao; PRB 496 (1994) 3982

[Warren 1990] B. E. Warren; X-ray diffraction, Dover publishing, Inc., Nueva York (1990) pág. 253

[Yasuna A] K. Yasuna, A. Otsuki, K. N. Ishihara, P.H. Shingu; "Relationship between giant magnetoresistance and microstructure in $\mathrm{Co}-\mathrm{Cu}$ prepared by mechanical alloying"; resultados no publicados

[Yasuna 1997] K. Yasuna, M. Terauchi, A. Otsuki, K. N. Ishihara y P. H. Shingu; J. Appl. Phys. 825 (1997) 2435

[Yavari 1992] A. R. Yavari, P.J. Desré, y T. Benameur; Phys. Rev. Lett. 68 (1992) 2235

[Yavari 1993] A. R. Yavari; Phys. Rev. Lett 7022 (1993) 3522

[Yermakov 1995] A. Ye. Yermakov, M. A. Uimin, A. V. Shangurov, A. V. Zarubin, Ye. V. Chechetkin, A. K. Shtolz, V. V. Kondratyev, G. N. Konygin, Ye. V. Yelsukov, S. Enzo, P.P. Macrí, R. Frattini y N. Cowlam: presentación al International Symposium on Metastable, Mechanically Alloyed, Nanocrystalline Materials, Québec 1995.

[Xiao 1992] J. Q. Xiao, J. S. Jiang y C. L. Chien, Phys. Rev. Lett., 68 (1992) 3749.

[Ziman 1967] Electrons and phonons, Ziman, Oxford, Londres (1967), pág. 337 